\title{
Fully covering the MSSM Higgs sector at the LHC
}

\author{
A. Djouadi, ${ }^{a}$ L. Maiani, ${ }^{b}$ A. Polosa, ${ }^{b}$ J. Quevillon ${ }^{c}$ and V. Riquer ${ }^{b}$ \\ ${ }^{a}$ Laboratoire de Physique Théorique d'Orsay, Université Paris XI and CNRS, \\ F-91405 Orsay, France \\ ${ }^{b}$ Department of Physics and INFN, Università di Roma Sapienza, \\ Piazzale Aldo Moro 5, I-00185 Roma, Italia \\ ${ }^{c}$ Theoretical Particle Physics and Cosmology Group, Physics Department, King's College London, \\ London WC2R 2LS, U.K. \\ E-mail: abdelhak.djouadi@th.u-psud.fr, luciano.maiani@roma1.infn.it, \\ antonio.polosa@roma1.infn.it, jeremie.quevillon@kcl.ac.uk, \\ veronica.riquer@cern.ch
}

Abstract: In the context of the Minimal Supersymmetric extension of the Standard Model (MSSM), we reanalyze the search for the heavier CP-even $H$ and CP-odd $A$ neutral Higgs bosons at the LHC in their production in the gluon-fusion mechanism and their decays into gauge and lighter $h$ bosons and into top quark pairs. We show that only when considering these processes, that one can fully cover the entire parameter space of the Higgs sector of the model. Indeed, they are sensitive to the low $\tan \beta$ and high Higgs mass ranges, complementing the traditional searches for high mass resonances decaying into $\tau$-lepton pairs which are instead sensitive to the large and moderate $\tan \beta$ regions. The complementarity of the various channels in the probing of the complete $\left[\tan \beta, M_{A}\right.$ ] MSSM parameter space at the previous and upcoming phases of the LHC is illustrated in a recently proposed simple and model independent approach for the Higgs sector, the $h \mathrm{MSSM}$, that we also refine in this paper.

Keywords: Supersymmetry Phenomenology

ARXiv EPrint: 1502.05653 


\section{Contents}

1 Introduction 1

2 The hMSSM approach $\quad 5$

3 MSSM Higgs production and decays at the LHC 12

$\begin{array}{ll}3.1 \text { Neutral Higgs decays } & 12\end{array}$

$\begin{array}{ll}3.2 \text { Neutral Higgs production } & 16\end{array}$

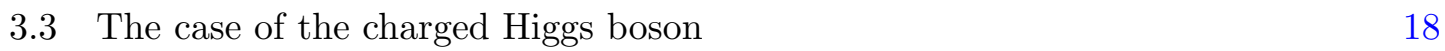

3.4 Impact of the SUSY spectrum and dark matter 21

4 The probing of the MSSM parameter space $\quad 27$

4.1 Interpretation of the fermionic Higgs decay modes in the hMSSM 27

4.2 Interpretation of the bosonic Higgs decay modes in the hMSSM 30

4.3 Summary of the constraints at $8 \mathrm{TeV}$ and projections for $14 \mathrm{TeV}$

4.4 Including the $p p \rightarrow H / A \rightarrow t \bar{t}$ channel 34

5 Conclusions $\quad 39$

\section{Introduction}

The probing of the electroweak symmetry breaking mechanism and the search for possible extensions of the Standard Model (SM) of particle physics has become the main mission of the CERN Large Hadron Collider (LHC). Among these extensions, Supersymmetry (SUSY) [1-3] is considered as the most appealing one as it addresses several shortcomings of the SM, including the problem of the large hierarchy between the Planck and electroweak scales. While the search for SUSY was unsuccessful at the first LHC run, the increase of the center of mass energy of the machine from $8 \mathrm{TeV}$ to the $14 \mathrm{TeV}$ level will significantly improve the sensitivity to the new particles that are predicted by the weak scale theory. These consists not only of the superpartners of the known fermions and gauge bosons but, also, of the additional Higgs bosons beyond the state with a mass of $125 \mathrm{GeV}$ that has been observed by the ATLAS and CMS collaborations in the first LHC phase [4, 5].

As a matter of fact, in low-energy SUSY scenarios, at least two Higgs doublet fields $H_{u}$ and $H_{d}$ are required to break the electroweak symmetry and to generate the isospin-up and down type fermion and the $W / Z$ boson masses. In the simplest scenario, the Minimal Supersymmetric Standard Model (MSSM), the spectrum consists of five states [6-10]: two charged $H^{ \pm}$, a CP-odd $A$ and two CP-even Higgs particles $h$ and $H$, with $h$ being the state observed at the LHC while $H$ is heavier as present LHC data is strongly indicating [11-13].

The phenomenology of the Higgs sector is described entirely by two input parameters, one Higgs mass that is usually taken to be that of the pseudoscalar $A$ boson $M_{A}$ and the 
ratio $\tan \beta$ of the vacuum expectation values of the two doublet fields, which is generally assumed to lie in the range $1 \lesssim \tan \beta \lesssim m_{t} / m_{b} \approx 60$. This is the case at tree-level where, for instance, the lightest $h$ boson mass is an output and is predicted to be $M_{h} \lesssim M_{Z}|\cos 2 \beta|$, i.e. $M_{h} \leq M_{Z}$ at high $\tan \beta$ for which $|\cos 2 \beta| \simeq 1$. However, this relation is violated since important radiative corrections, that introduce a dependence on many SUSY parameters, occur in this context [14-25]. It has been recently shown that, to a good approximation, the MSSM Higgs sector can be again parametrised using the two basic inputs $\tan \beta$ and $M_{A}$, provided that the crucial LHC information $M_{h} \simeq 125 \mathrm{GeV}$ is used [26-30].

It is known that two efficient channels can be used to directly search for the heavier MSSM Higgs particles at the LHC and probe part of the $\left[\tan \beta, M_{A}\right]$ parameter space. ${ }^{1}$ The first one is the search for light charged Higgs bosons that would emerge from the decays of the copiously produced top quarks and would decay almost exclusively into a $\tau$ lepton and its associated neutrino for $\tan \beta \gtrsim 1$. For almost all values of $\tan \beta$, the latest ATLAS [33] and CMS [34] results now practically rule out the mass range $M_{H^{ \pm}} \lesssim 160 \mathrm{GeV}$, which approximately corresponds to $M_{A} \lesssim 140 \mathrm{GeV}$ in the MSSM. The second efficient channel is the search for high mass resonances decaying into $\tau$-lepton pairs, which would be the signature of the production of the heavy neutral $H / A$ states and their decay into $\tau$ leptons. The rates for this channel can be very large at high $\tan \beta$ values, as a consequence of the strong enhancement of the $H / A$ couplings to bottom quarks and $\tau$-leptons. This process is particularly favored as, for a heavy enough $A$ boson, one has the mass degeneracy relation $M_{H} \approx M_{A}$ that in practice leads to search for a single resonance and allows to combine the rates for $A$ and $H$ production. The most recent ATLAS [35] and CMS [36] results with the data collected at the first LHC phase, exclude at the $95 \%$ confidence level (CL) a significant portion of the $\left[\tan \beta, M_{A}\right]$ plane for sufficiently high $\tan \beta$ values.

Except in the narrow mass range $M_{Z} \lesssim M_{A} \lesssim 140 \mathrm{GeV}$, where the lower value corresponds to the exclusion limit from negative Higgs searches at the LEP collider $[37,38]$ and the upper one is due to the present limit from charged Higgs boson searches at the LHC (which can be straightforwardly interpreted in the $\left[\tan \beta, M_{H^{ \pm}}\right]$parameter space as the $H^{ \pm}$properties depend only on these two parameters in the low mass range), the low $\tan \beta$ region of the MSSM has not been considered so far by the experimental collaborations. The reason is that in the benchmark scenarios that are used to interpret the various experimental limits on the cross sections times branching ratios in the context of the MSSM [39, 40], the SUSY-breaking scale is usually set to relatively low values, $M_{S} \approx 1 \mathrm{TeV}$, that do not allow for a heavy enough $h$ state at too low $\tan \beta$. Indeed, the radiative corrections to the mass $M_{h}$ depend logarithmically on the scale $M_{S}$ and, for instance, one cannot obtain a value $M_{h} \approx 125 \mathrm{GeV}$ for $\tan \beta \lesssim 3-5$ in the MSSM, even if one favorably tunes the other SUSY parameters that enter the loop radiative corrections, in particular the stop mixing parameter $X_{t}$ which also plays an important role in this context. This is the case of the

\footnotetext{
${ }^{1}$ Of course, there are also indirect limits on the MSSM parameter space, in particular from the measurement of the couplings of the observed $h$ particle at the LHC; see for instance refs. [26, 31, 32]. These limits are nevertheless slightly model dependent as, for instance, they can be affected by SUSY particle contributions to the $h$ production and decay rates. These indirect limits, that exclude low values of the $A, H, H^{ \pm}$ masses, are complementary to those from the direct Higgs searches on which we will focus in this paper.
} 
so-called maximal mixing or $M_{h}^{\max }$ scenario which is defined such that the value of $M_{h}$ is maximized, i.e. for a stop mixing parameter $X_{t} \simeq \sqrt{6} M_{S}$ in the dimensional reduction scheme [39]. The situation is even worse for different values of the $X_{t}$ parameter.

In fact, in most of the $\left[\tan \beta, M_{A}\right]$ parameter space, the measured value $M_{h} \approx 125 \mathrm{GeV}$, which should be now considered as an essential information on the model, is not satisfied in the $M_{h}^{\max }$ benchmark scenario with $M_{S}=1 \mathrm{TeV}$ nor in the alternative benchmark scenarios that are presently used to interpret the experimental searches in the context of the MSSM. If one allows for an uncertainty of a say $3 \mathrm{GeV}$ in the determination of the $h$ mass in the MSSM, from unknown higher order contributions for instance [41-44], the situation is acceptable if the $h$ mass is confined in the range $122 \mathrm{GeV} \lesssim M_{h} \lesssim 128 \mathrm{GeV}$. Nevertheless, it remains annoying that for each point of the $\left[\tan \beta, M_{A}\right]$ parameter space, one has a different $M_{h}$ value in these benchmark scenarios.

A straightforward and easily implementable solution to this problem has been proposed in refs. [26-30]: if the experimental constraint $M_{h} \approx 125 \mathrm{GeV}$ is enforced, one in fact removes the dependence of the Higgs sector on the dominant radiative correction and, hence, on the additional SUSY parameters, in particular $M_{S}$ and $X_{t}$. One can again parametrise the MSSM Higgs sector using only the two basic inputs $\tan \beta$ and $M_{A}$, exactly like it was the case at tree-level. The masses of the heavier $H$ and $H^{ \pm}$states as well as the mixing angle $\alpha$ in the $\mathrm{CP}$-even sector are given by very simple expressions in terms of $\tan \beta$ and $M_{A}$ with the constraint $M_{h}=125 \mathrm{GeV}$. It was shown that this approximation is very good in most of the MSSM parameter space that is currently accessible at the LHC, even when subleading radiative corrections are also considered [26].

In this minimal and almost model independent approach, called the $h$ MSSM in ref. [26], one has access to the entire $\left[\tan \beta, M_{A}\right]$ parameter space without being in conflict with the LHC data, as the information $M_{h}=125 \mathrm{GeV}$ is taken into account from the very beginning (this is not always the case for the Higgs couplings which conflict with the measured ones at low $M_{A}$ ). In particular, the low $\tan \beta$ region can naturally be accessed, but at the expense of assuming a very high SUSY scale $M_{S}$. The reason is that at $\tan \beta$ values too close to unity, the tree-level $h$ mass becomes very small, $M_{h} \approx M_{Z}|\cos 2 \beta| \rightarrow 0$. To increase $M_{h}$ to $\approx 125 \mathrm{GeV}$, the radiative corrections that grow logarithmically with $M_{S}$ need to be maximized and hence, a very large scale, $M_{S} \gtrsim \mathcal{O}(100) \mathrm{TeV}$ for $\tan \beta \lesssim 2$, is required.

The low $\tan \beta$ region can be directly probed by the search for the heavier $H / A$ (and eventually $H^{ \pm}$) states and for relatively low Higgs masses, $M_{H} \approx M_{A} \lesssim 350 \mathrm{GeV}$, two ways have been suggested. First, one can use the same constraint discussed above from the search of resonances decaying into $\tau$-lepton pairs [30]. Indeed, the rates for $A / H$ production are appreciable at low $\tan \beta$ as the dominant process, the gluon-fusion mechanism, is now primarily mediated by loops of top quarks that have significant couplings to the $H / A$ bosons; at the same time, the decay of at least the $A$ boson into $\tau \tau$ pairs has a still appreciable rate. The second way is to reinterpret the existing ATLAS and CMS exclusion limits from the search for a heavy SM-like Higgs boson decaying into a pair of massive gauge bosons $[45,46]$ in the context of the MSSM. At low $\tan \beta$ and not too large $M_{H}$ values for which we are not yet in the decoupling regime with a vanishing $H$ coupling to massive gauge bosons, the rates for the decays $H \rightarrow V V$ with $V=W, Z$, as well as for 
$g g \rightarrow H$ production, are still significant. In addition, searches for the resonant $h h[47,48]$ and $h Z$ [49] topologies have been performed at the LHC with the available $25 \mathrm{fb}^{-1}$ data at $\sqrt{s}=7+8 \mathrm{TeV}$, and one can reinterpret them in the context of the MSSM where the production cross section for $g g \rightarrow H / A$ and the branching ratios for the decay modes $H \rightarrow h h$ and $A \rightarrow Z h$ below the $t \bar{t}$ threshold can be substantial; see refs. [30, 50-56].

The two types of searches mentioned above, with results that were preliminary and obtained with a subset of the LHC data collected at $\sqrt{s}=7+8 \mathrm{TeV}$, have been used in ref. [30] to set constraints on the $\left[\tan \beta, M_{A}\right]$ plane; excluded regions have been delineated using some approximations and extrapolations. In the present paper, we update this discussion first by using the latest ATLAS and CMS results, especially the final $H / A \rightarrow \tau^{+} \tau^{-}$and $t \rightarrow b H^{+} \rightarrow b \tau \nu$ analyses [33-36] as well as heavy SM Higgs searches in the $H \rightarrow W W, Z Z$ channels [45, 46], with the full set of $25 \mathrm{fb}^{-1}$ data collected in the first LHC phase. In addition, constraints from more appropriate analyses in the $A \rightarrow h Z$ and $H \rightarrow h h$ topologies where the resonant case has now been considered [47-49] will be included. We will then extrapolate these results to estimate the sensitivity of the $14 \mathrm{TeV}$ LHC run, with at least an order of magnitude higher integrated luminosity than the one accumulated so far.

Above the $t \bar{t}$ threshold, i.e. for $M_{A, H} \gtrsim 350 \mathrm{GeV}$, the previously discussed search channels will have little relevance at low $\tan \beta$ values as, because their couplings to $b$ quarks and $\tau$ leptons are not enhanced anymore, the heavier $H$ and $A$ bosons will dominantly decay into $t \bar{t}$ pairs, the top-quark Yukawa coupling $\propto m_{t} / \tan \beta$ becoming then large. As already mentioned, the main Higgs production channel will be the gluon-fusion process $g g \rightarrow H / A$ in which the top quark loop generates the dominant contribution. We will see that the production times the decay rates in the processes $g g \rightarrow H / A \rightarrow t \bar{t}$ are indeed substantial in a large part of the MSSM parameter space. We perform a naive estimate of the sensitivity that can be achieved in the search for $t \bar{t}$ resonances, a sensitivity that could allow to probe a significant part of the low $\tan \beta$ region of the MSSM, complementing the searches for $\tau^{+} \tau^{-}$resonances that are instead sensitive to the high $\tan \beta$ region.

The main message of the present paper is that combining the searches for heavy resonances decaying into $\tau^{+} \tau^{-}$and $t \bar{t}$ pairs, and including the $H \rightarrow W W, Z Z, h h$ and $A \rightarrow h Z$ channels at $M_{A} \lesssim 350 \mathrm{GeV}$, one can possibly probe the entire $\left[\tan \beta, M_{A}\right.$ ] MSSM plane (and not only the high-tan $\beta$ region as is presently the case) up to large pseudoscalar Higgs masses; $M_{A}$ values close to $1 \mathrm{TeV}$ could be reached for any $\tan \beta$ with $\approx 3000 \mathrm{fb}^{-1}$ data at the LHC with $\sqrt{s}=14 \mathrm{TeV}$. This can be done in a model independent way and without relying on any additional theoretical assumption or indirect experimental constraint.

The paper is organized as follows. We first summarise our simple parametrisation of the MSSM Higgs sector, further discussing and refining the $h$ MSSM approach. In section 3 , we discuss the heavier Higgs production and decay rates focusing on low $\tan \beta$ and summarise the possible impact of superparticles. In section 4 , the probing of the $\left[\tan \beta, M_{A}\right]$ MSSM parameter space is discussed when all the search channels, including a projection for the the $H / A \rightarrow t \bar{t}$ channel, are combined first at $\sqrt{s}=7+8 \mathrm{TeV}$ with $25 \mathrm{fb}^{-1}$ data and then at $\sqrt{s}=14 \mathrm{TeV}$ and higher luminosities. A brief conclusion is given in a final section. 


\section{The hMSSM approach}

In this section, we briefly describe the $h \mathrm{MSSM}$ introduced in ref. [26], an approach that allows to parameterize the CP-conserving MSSM Higgs sector in a simple (as only two inputs are needed) and "model independent" (in the sense that we do not consider or fix any other SUSY parameter) way, using the information that the lightest $h$ boson has a mass $M_{h} \approx 125 \mathrm{GeV}$. The approach is based on several assumptions that we first summarize.

The first basic assumption of the $h \mathrm{MSSM}$ is that in the basis $\left(H_{d}, H_{u}\right)$ of the two MSSM Higgs doublet fields that break electroweak symmetry, the CP-even $h$ and $H$ mass matrix can be simply written in terms of the $Z$ and $A$ boson masses and the angle $\beta$ as

$$
M_{\Phi}^{2}=\left(\begin{array}{cc}
M_{Z}^{2} \cos ^{2} \beta+M_{A}^{2} \sin ^{2} \beta & -\left(M_{Z}^{2}+M_{A}^{2}\right) \sin \beta \cos \beta \\
-\left(M_{Z}^{2}+M_{A}^{2}\right) \sin \beta \cos \beta & M_{Z}^{2} \sin ^{2} \beta+M_{A}^{2} \cos ^{2} \beta
\end{array}\right)+\left(\begin{array}{cc}
\Delta \mathcal{M}_{11}^{2} \Delta \mathcal{M}_{12}^{2} \\
\Delta \mathcal{M}_{12}^{2} \Delta \mathcal{M}_{22}^{2}
\end{array}\right)
$$

in which the radiative corrections are introduced through a $2 \times 2$ general matrix $\Delta \mathcal{M}_{i j}^{2}$. This is the usual starting point of the analyses of the neutral MSSM Higgs sector [9] and the calculation of the Higgs masses and couplings including radiative corrections and in which the SUSY scale, taken to be the geometric average of the two stop masses, $M_{S}=\sqrt{m_{\tilde{t}_{1}} m_{\tilde{t}_{2}}}$, can be as high as a few TeV. However, if $M_{S}$ is orders of magnitude higher than the TeV scale, the evolution from this high scale down to the electroweak scale might mix the quartic couplings of the MSSM Higgs sector in a non trivial way, such that the structure of the mass matrix at the low energy scale is different from the one given in eq. (2.1).

In the $h \mathrm{MSSM}$, we assume that the form of the CP-even Higgs mass matrix is as given above even at the very high SUSY scales, $M_{S} \gtrsim \mathcal{O}(100 \mathrm{TeV})$ that, as it will be seen later, are needed to consider $\tan \beta$ values close to unity. ${ }^{2}$

A second basic assumption of the hMSSM is that in the $2 \times 2$ matrix above for the radiative corrections, only the $\Delta \mathcal{M}_{22}^{2}$ entry is relevant, $\Delta \mathcal{M}_{22}^{2} \gg \Delta \mathcal{M}_{11}^{2}, \Delta \mathcal{M}_{12}^{2}$. In this case, one can simply trade $\Delta \mathcal{M}_{22}^{2}$ for the by now known mass value $M_{h}=125 \mathrm{GeV}$ using

$$
\Delta \mathcal{M}_{22}^{2}=\frac{M_{h}^{2}\left(M_{A}^{2}+M_{Z}^{2}-M_{h}^{2}\right)-M_{A}^{2} M_{Z}^{2} \cos ^{2} 2 \beta}{M_{Z}^{2} \cos ^{2} \beta+M_{A}^{2} \sin ^{2} \beta-M_{h}^{2}} .
$$

This assumption is valid in most cases as the by far dominant radiative correction from the stop-top sector that is quartic in the top quark mass, enters only in this entry [14-18]:

$$
\Delta \mathcal{M}_{22}^{2} \sim \frac{3 v^{2} \sin ^{2} \beta}{8 \pi^{2}} \lambda_{t}^{4}\left[\log \frac{M_{S}^{2}}{\bar{m}_{t}^{2}}+\frac{X_{t} A_{t}}{M_{S}^{2}}\left(1-\frac{X_{t} A_{t}}{12 M_{S}^{2}}\right)\right]
$$

which depends on, besides $M_{S}$, the stop mixing parameter given by $X_{t}=A_{t}-\mu / \tan \beta$ with $A_{t}$ the stop trilinear coupling and $\mu$ the higgsino mass parameter. $\lambda_{t}=\sqrt{2} \bar{m}_{t} /(v \sin \beta)$ is the top Yukawa coupling with $v$ the standard vacuum expectation value $v \approx 246 \mathrm{GeV}$, and $\bar{m}_{t}$ the running $\overline{\mathrm{MS}}$ top quark mass to account for the leading two-loop corrections in a renormalisation group (RG) improved approach.

\footnotetext{
${ }^{2}$ The validity of this approximation is currently studied by the LHC Higgs cross section working group [57]. Preliminary results in an effective two Higgs doublet model that is RG improved to resum the large logarithms involving $M_{S}$, have been given in refs. $[58,59]$ and a more refined analysis is under way $[57,60]$.
} 
The maximal value of the $h$ mass, $M_{h}^{\max }$ is given in the approximation above by

$$
M_{h}^{2} \rightarrow M_{Z}^{2} \cos ^{2} 2 \beta+\Delta \mathcal{M}_{22}^{2}
$$

and is obtained for the following choice of parameters [39]: a decoupling regime with a heavy pseudoscalar $A$ boson, large enough $\tan \beta$ values that allow to maximize the treelevel term $M_{Z}^{2} \cos ^{2} 2 \beta \rightarrow M_{Z}^{2}$, heavy stop squarks with a sufficiently large $M_{S}$ value to enhance the $\operatorname{logarithmic}$ correction $\log \left(M_{S}^{2} / \bar{m}_{t}^{2}\right)$ and, finally, a stop mixing parameter such that $X_{t}=\sqrt{6} M_{S}$, the so-called maximal mixing scenario that maximizes the stop loops and hence $M_{h}$. If the SUSY parameters are optimized as above, the maximal $M_{h}$ value can reach the level of $M_{h}^{\max } \approx 130 \mathrm{GeV}$ for $M_{S}$ of the order of the TeV scale, a range that is in general assumed in order to avoid a too large fine-tuning in the model. However, if $\tan \beta$ is small, the tree-level contribution $M_{Z}^{2} \cos ^{2} 2 \beta$ to the $h$ mass squared becomes small as $|\cos 2 \beta| \rightarrow 0$, thus requiring a substantial correction $\Delta \mathcal{M}_{22}^{2}$ to obtain a sufficiently large $M_{h}$. To achieve this, eq. (2.3) shows that one has to substantially increase $M_{S}$.

In ref. [26], the approximation $\Delta \mathcal{M}_{11}^{2}, \Delta \mathcal{M}_{12}^{2} \ll \Delta \mathcal{M}_{22}^{2}$ has been checked in various scenarios and found to be rather good if $M_{S}$ much larger than the other soft-SUSY breaking parameters that enter the subleading radiative corrections, such as the higgsino mass $\mu$ and the sbottom trilinear coupling $A_{b}$ or more generally the sbottom mixing parameter $X_{b}=A_{b}-\mu \tan \beta$. This assumption should be particularly justified at low and moderate $\tan \beta$ values where first, the bottom-Yukawa coupling $\lambda_{b}=\sqrt{2} \bar{m}_{b} /(v \cos \beta)$ is not strongly enhanced. In the approach of ref. [10] to parameterize the correction matrix of eq. (2.1) including the dominant corrections from the stop and sbottom sectors (and which has been used in ref. [26] to check this second hMSSM assumption), the entries $\Delta \mathcal{M}_{11}^{2}$ and $\Delta \mathcal{M}_{12}^{2}$ of the mass matrix when $\lambda_{b}$ is set to zero are simply given at lowest order by $[9,17,18]$

$$
\Delta \mathcal{M}_{11}^{2} \simeq-\frac{v^{2} \sin ^{2} \beta}{32 \pi^{2}} \lambda_{t}^{4} \times \frac{\mu^{2} X_{t}^{2}}{M_{S}^{4}}, \quad \Delta \mathcal{M}_{12}^{2} \simeq-\frac{v^{2} \sin ^{2} \beta}{32 \pi^{2}} \lambda_{t}^{4} \times \frac{\mu X_{t}}{M_{S}^{2}}\left(6-X_{t} A_{t} / M_{S}^{2}\right) .
$$

They are proportional to $\mu / M_{S}$ and hence, are small if $|\mu| \lesssim M_{S}$. Note that from the expressions above one can see that the two entries $\Delta \mathcal{M}_{11}^{2}$ and $\Delta \mathcal{M}_{12}^{2}$ are small not only for $M_{S} \gg|\mu|$, but also when stop mixing is small, $M_{S} \gg X_{t}$. For moderate $\tan \beta$ (and also at large $\tan \beta$ if the sbottom corrections can still be neglected), one has $A_{t} \approx X_{t}$ and the off-diagonal entry is further suppressed for maximal $X_{t}=\sqrt{6} M_{S}$. Thus, the approximation of retaining only the entry $\Delta \mathcal{M}_{22}^{2}$ for the radiative corrections should be good at least at low $\tan \beta$ where a very high SUSY scale is required to obtain a heavy enough $h$ state, suggesting that one naturally has $M_{S} \gg|\mu|$ and eventually also $M_{S} \gg X_{t}$.

In this $h \mathrm{MSSM}$ approach the mass of the neutral CP even $H$ particle and the mixing angle $\alpha$ that diagonalises the $h, H$ states, will be given by the extremely simple expressions

$$
\begin{aligned}
M_{H}^{2} & =\frac{\left(M_{A}^{2}+M_{Z}^{2}-M_{h}^{2}\right)\left(M_{Z}^{2} \cos ^{2} \beta+M_{A}^{2} \sin ^{2} \beta\right)-M_{A}^{2} M_{Z}^{2} \cos ^{2} 2 \beta}{M_{Z}^{2} \cos ^{2} \beta+M_{A}^{2} \sin ^{2} \beta-M_{h}^{2}} \\
\alpha & =-\arctan \left(\frac{\left(M_{Z}^{2}+M_{A}^{2}\right) \cos \beta \sin \beta}{M_{Z}^{2} \cos ^{2} \beta+M_{A}^{2} \sin ^{2} \beta-M_{h}^{2}}\right)
\end{aligned}
$$

in terms of the inputs $M_{A}$, $\tan \beta$ and the mass of the lightest $h$ state $M_{h}=125 \mathrm{GeV}$. 
The mass of the charged Higgs boson is simply given by the tree-level relation

$$
M_{H^{ \pm}}^{2}=M_{A}^{2}+M_{W}^{2}
$$

as the SUSY radiative corrections in this particular case are known to be very small in general. According to ref. [61] where a detailed analysis of the radiative corrections has been recently performed, the leading one-loop correction to $M_{H^{+}}^{2}$ reads when expanding in powers of the SUSY scale as it is justified at low $\tan \beta$

$$
\Delta M_{H^{ \pm}}^{2}=-\frac{3 \alpha}{16 \pi \sin ^{2} \theta_{W}} \frac{m_{t}^{4}}{M_{W}^{2} \sin ^{2} \beta} \frac{\mu^{2}}{M_{S}^{2}}+\mathcal{O}\left(1 / M_{S}^{4}\right)
$$

and is therefore very small for $M_{S} \gg|\mu|$. In fact, even for $M_{S} \approx|\mu|$ one obtains $\Delta M_{H^{ \pm}}^{2} \approx$ $-10^{3}(250) \mathrm{GeV}^{2}$ for $\tan \beta \approx 1(\tan \beta \gg 1)$ and, hence, a relative correction $\left|\Delta M_{H^{ \pm}} / M_{H^{ \pm}}\right|$ that is only about $5 \%(1 \%)$ for $M_{H^{ \pm}} \approx 100 \mathrm{GeV}$ and negligibly small for higher $H^{ \pm}$masses. Hence, retaining only the tree-level relation eq. (2.7) as done in the $h$ MSSM should be a very good approximation in this case.

A third assumption of the hMSSM is that all couplings of the Higgs particles to fermions and gauge bosons are given in terms of $\tan \beta$ and the mixing angle $\alpha$ only and, hence, the entire phenomenology of the Higgs particles is determined when the two inputs $\tan \beta$ and $M_{A}$ are fixed. This means that possible corrections not incorporated in the mixing angle $\alpha$, such as direct vertex corrections, are assumed to have a small impact. ${ }^{3}$ In particular, the couplings of the neutral Higgs bosons, collectively denoted by $\Phi$, to up and down-type fermions and to massive gauge bosons (including the coupling of two Higgs and one gauge bosons) when normalized to the SM-Higgs couplings, are simply given by:

$\begin{array}{ccccc}\Phi & g_{\Phi \bar{u} u} & g_{\Phi \bar{d} d} & g_{\Phi V V} & g_{\Phi A Z} / g_{\Phi H^{+} W^{-}} \\ h & \cos \alpha / \sin \beta & -\sin \alpha / \cos \beta & \sin (\beta-\alpha) & \propto \cos (\beta-\alpha) \\ H & \sin \alpha / \sin \beta & \cos \alpha / \cos \beta & \cos (\beta-\alpha) & \propto \sin (\beta-\alpha) \\ A & \cot \beta & \tan \beta & 0 & \propto 0 / 1 .\end{array}$

The trilinear self-couplings among the Higgs bosons are also given in terms of $\beta$ and $\alpha$. This is clearly the case at tree-level but, to a good approximation, it remains true when radiative corrections are incorporated. Indeed, besides the corrections that affect the angle $\alpha$ as discussed above, the trilinear couplings receive direct corrections whose dominant component turns out to be simply the one that appears in the correction matrix $\Delta \mathcal{M}^{2}$ and hence, the correction $\Delta \mathcal{M}_{22}^{2}$ of eq. (2.2) [62-66]. Thus, the trilinear MSSM Higgs couplings are also fixed in terms of $M_{A}, \tan \beta$ and $M_{h}$ to a good approximation. In units of $\lambda_{0}=-i M_{Z}^{2} / v$, the $H h h$ and $h h h$ self-couplings, which are the only ones that will matter for LHC phenomenology, will be then given by

$$
\begin{aligned}
& \lambda_{h h h}=3 \cos 2 \alpha \sin (\beta+\alpha)+3 \frac{\Delta \mathcal{M}_{22}^{2}}{M_{Z}^{2}} \frac{\cos \alpha}{\sin \beta} \cos ^{2} \alpha \\
& \lambda_{H h h}=2 \sin 2 \alpha \sin (\beta+\alpha)-\cos 2 \alpha \cos (\beta+\alpha)+3 \frac{\Delta \mathcal{M}_{22}^{2}}{M_{Z}^{2}} \frac{\sin \alpha}{\sin \beta} \cos ^{2} \alpha .
\end{aligned}
$$

\footnotetext{
${ }^{3}$ The direct corrections can be particularly important at high $\tan \beta$ in the bottom-quark Yukawa coupling, as is the case of the so-called $\Delta_{b}$ correction which can be large if $|\mu| \tan \beta \gg M_{S}$. We will show in the next section that even in this case, the impact of this direct correction is limited in LHC phenomenology.
} 
We note that at least for the $h h h$ self-coupling, one should incorporate the radiative corrections in the same approximation that has been used for the Higgs masses. This would be the only way to achieve a consistent decoupling limit and to make that the $\lambda_{h h h}$ selfcoupling indeed reaches the SM value in this limit, $\lambda_{h h h}=3 M_{h}^{2} / M_{Z}^{2}$ for $\alpha=\beta-\frac{\pi}{2}$. For the sake of consistency, one should include the radiative corrections to the other self-couplings in the same approximation as for $\lambda_{h h h}$. This then fully justifies the choice that we adopt in the $h \mathrm{MSSM}$ and the expression of eq. (2.10) for the Higgs self-couplings.

From the discussion above, one can conclude that the $h \mathrm{MSSM}$ approach has two very interesting aspects: its economy as only two input parameters are needed to describe the entire MSSM Higgs sector and its simplicity, as the Higgs masses and couplings are given by the very simple relations eqs. (2.6)-(2.10). This would allow to considerably simplify phenomenological analyses of the MSSM Higgs sector which, because of the large number of SUSY parameters to be taken into account, rely up to now either on large scans of the parameter space or resort to benchmark scenarios in which most of these parameters are fixed. Nevertheless, the most interesting aspect of the $h \mathrm{MSSM}$ is that it easily allows to describe scenarios with large values of the SUSY scale, $M_{S} \gg 1 \mathrm{TeV}$, but weak-scale masses for the extended Higgs sector.

Because of the large $\log \left(M_{S} / m_{h}\right)$ that occur, the high SUSY scale scenarios are notoriously difficult to analyze and, before resuming the large logarithms, the MSSM Higgs spectrum could not be calculated in a reliable way. Until very recently, this was the case of the numerical tools that deal with the MSSM, such as the renormalisation group program Suspect [67] or the program FeynHiggs [68] that is more specialized on the MSSM Higgs sector, which were not reliable at too high $M_{S}$ outside the decoupling regime. A new version of FeynHiggs in which some partial resummation of the large logarithmic terms is performed has become available and allows to address low $\tan \beta$ values in a somewhat reliable way ${ }^{4}$ (albeit with $M_{h}$ values still below $M_{h} \approx 125 \mathrm{GeV}$ at $\tan \beta \lesssim 2$ ). The $h$ MSSM approach is currently being implemented in an updated version of the program Suspect.

An immediate advantage of the $h \mathrm{MSSM}$ is that it re-opens the possibility of studying the MSSM low $\tan \beta$ region [30], which was for a long time overlooked. Indeed, as only SUSY scales of the order of $M_{S} \approx 1 \mathrm{TeV}$ were assumed in the analyses performed in the past, one always had a too light $h$ boson with a mass below the limit $M_{h} \gtrsim 114 \mathrm{GeV}$ derived from the negative searches of a SM-like $h$ boson at the LEP2 collider [37, 38]. For a scale $M_{S}=1 \mathrm{TeV}$, the possibilities $\tan \beta \lesssim 3$ and $\tan \beta \lesssim 10$ were excluded for, respectively the maximal-mixing scenario $X_{t}=\sqrt{6} M_{S}$ and the no-mixing scenario $X_{t}=0$. The situation became worse with the observation of the $h$ state at the LHC and the determination that its mass is $M_{h} \approx 125 \mathrm{GeV}$, i.e. well beyond the LEP limit. In fact, for $M_{S} \approx 1 \mathrm{TeV}$, this

\footnotetext{
${ }^{4}$ This new version of the program FeynHiggs has been used to perform the following comparison [57]: for a $\left(\tan \beta, M_{A}\right)$ set and given other MSSM inputs (those of the $M_{h}^{\max }$ scenario for instance), one calculates $\alpha, M_{H}$ and $M_{h}$ using FeynHiggs and with the specific value obtained for $M_{h}$ (which is in general $M_{h} \neq$ $125 \mathrm{GeV}$ ), one recalculates the $h \mathrm{MSSM}$ values of $\alpha$ and $M_{H}$. The relative difference between FeynHiggs and the $h \mathrm{MSSM}$ was found to be extremely small in the entire $\left[\tan \beta, M_{A}\right]$ plane. Even at very low $M_{A}$ and $\tan \beta$ values, the outputs for $M_{H}$ and $\alpha$ differ by less than $1 \%$. This proves once more that the second assumption of the $h \mathrm{MSSM}$, i.e. that one can consider only the $\Delta M_{22}$ radiative correction, is fully justified. We thank Pietro Slavich, who originally performed this comparison, for a discussion on this issue.
} 


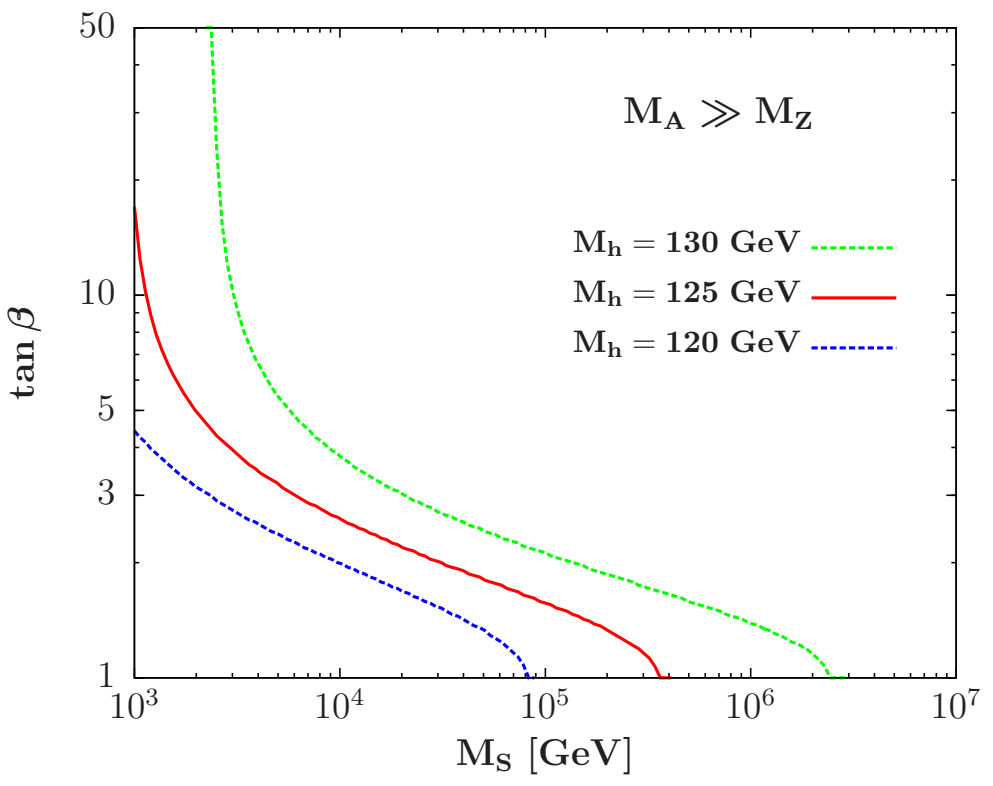

Figure 1. Contour plots in the $\left[\tan \beta, M_{S}\right]$ plane where the there values $M_{h}=120,125$ and $130 \mathrm{GeV}$ are obtained; the decoupling limit and maximal stop mixing are assumed. The SM inputs are $m_{t}=173 \mathrm{GeV}$ and $\alpha_{S}=0.118$ for the top quark mass and strong coupling constant [37].

relatively large $M_{h}$ value cannot be reached in a large part of the $\left[\tan \beta, M_{A}\right.$ ] parameter space that is being explored at the LHC in the search for the additional Higgs bosons.

Nonetheless, fixing the SUSY scale at $M_{S} \approx 1 \mathrm{TeV}$ is a very strong theoretical assumption and is currently challenged not only by the measured $M_{h}$ [70-79] but also by direct sparticles searches at the LHC [80], especially in constrained MSSM scenarios. In the search for the MSSM Higgs bosons at the LHC, one would like to avoid any such assumption and interpret the experimental results, for instance imposing the relevant experimental constraints in the absence of any evidence, in a way that is as model-independent as possible. The $h$ MSSM approach, as no assumption on the SUSY scale nor on any other SUSY parameter (except eventually that they should be smaller than $M_{S}$ ) is made, is more suitable in this respect. In fact, one is considering simply in this case a two-Higgs doublet model of type II [69] where the MSSM relations eqs. (2.6)-(2.7) are enforced; the superparticles are assumed to be too heavy to have an impact on the Higgs sector (as it will be shown to be generally the case in the next section). The only price to pay is that when the very low $\tan \beta$ region is addressed, one is implicitly considering a very large SUSY-breaking scale, making the MSSM a very unnatural and fine-tuned scenario.

To illustrate this feature, we display in figure 1 contours in the $\left[\tan \beta, M_{S}\right]$ plane in which one obtains the value $M_{h}=125 \mathrm{GeV}$ for the $h$ mass, as well as $M_{h}=120$ and $130 \mathrm{GeV}$. The latter examples are when one assumes that a possible mass shift of $\Delta M_{h}=5 \mathrm{GeV}$ is missing from unaccounted for subleading corrections (e.g. the contributions of the charginos and neutralinos that we have ignored here) or unknown higher order terms (a theoretical uncertainty of $\approx 3 \mathrm{GeV}$ in the determination of $M_{h}$ is usually assumed [41-44]). The limit $M_{A} \approx M_{S}$ and maximal stop mixing $X_{t}=\sqrt{6} M_{S}$ are assumed. 

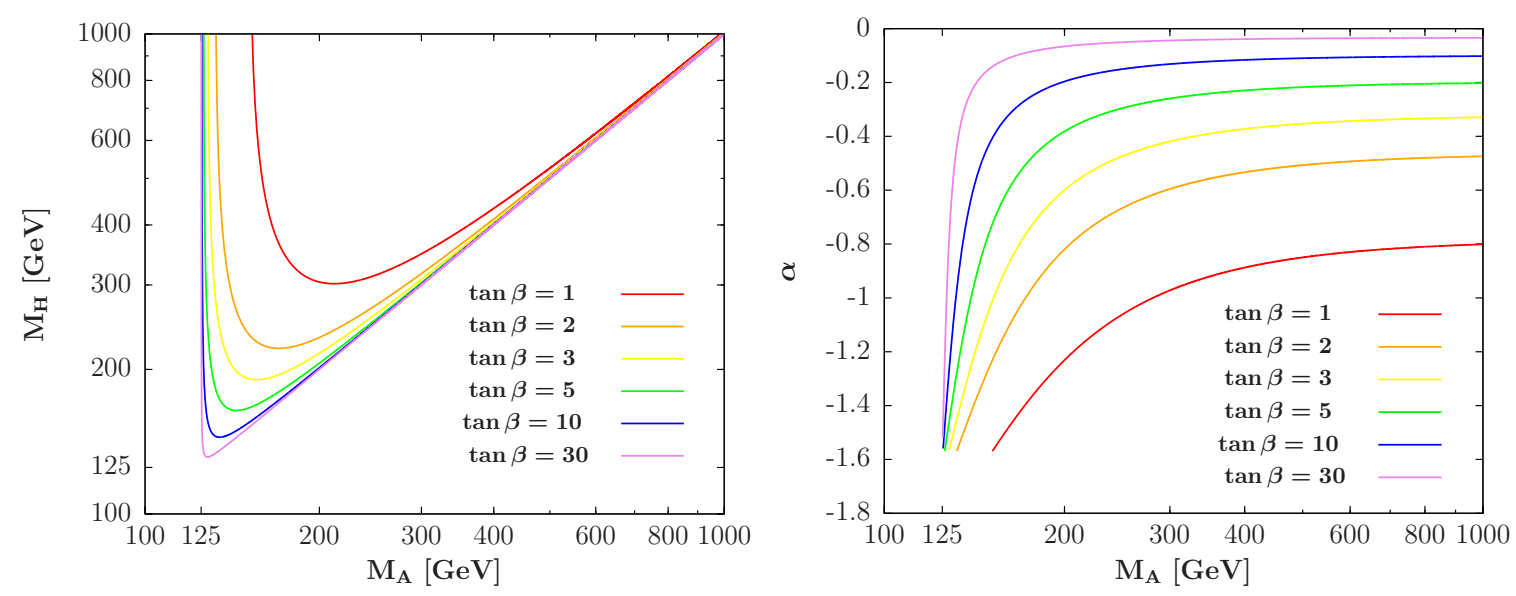

Figure 2. The CP-even $H$ boson mass (left) and the mixing angle $\alpha$ (right) in the $h \mathrm{MSSM}$ as a function of $M_{A}$ for representative values of $\tan \beta$; the $h$ mass is fixed to the value $M_{h}=125 \mathrm{GeV}$.

The figure has been in fact obtained from an analysis of the split-SUSY scenario where the large logarithms have been indeed resummed [81]. As can be seen, at high $\tan \beta, M_{S}$ values in the vicinity of the $\mathrm{TeV}$ scale can be accommodate while in the low $\tan \beta$ region, extremely large values of the SUSY scale $M_{S}$ are necessary to obtain $M_{h}=125 \mathrm{GeV}$.

This is particularly the case for $\tan \beta$ close to unity where a value $M_{S} \approx 400 \mathrm{TeV}$ is required for $\tan \beta=1$. The situation becomes even worse for the more natural small mixing situation $X_{t} \ll M_{S}$ and outside the decoupling regime when the tree-level $h$ mass is reduced by Higgs mixing. In both cases, huge $M_{S}$ values will be needed for $\tan \beta \approx 1$ to reach $M_{h}=125 \mathrm{GeV}$. For $\tan \beta \simeq 2$, the situation is less dramatic as in the configuration of figure 1 , only $M_{S} \approx 20 \mathrm{TeV}$ is needed to reach $M_{h}=125 \mathrm{GeV}$ (or the target value $M_{h} \approx 120 \mathrm{GeV}$ if uncertainties are included). We thus expect that our $h \mathrm{MSSM}$ approach should be valid down to $\tan \beta$ values as low as $\tan \beta \approx 2$, but in our analysis we will extend the validity of the approach to $\tan \beta$ values close to unity.

Let us now illustrate the values that one obtains for the two outputs of the $h \mathrm{MSSM}$, the CP-even $H$ mass $M_{H}$ and the mixing angle $\alpha$; the charged Higgs mass is simply given by eq. (2.7). These are shown in figure 2 as a function of $M_{A}$ for several representative $\tan \beta$ values, from unity to $\tan \beta=30$. One can see that at sufficiently high $\tan \beta$ values, $\tan \beta \gtrsim 10, M_{H}$ becomes very close to $M_{A}$ and the angle $\alpha$ close to $\beta-\frac{1}{2} \pi$, as soon as the pseudoscalar mass becomes larger than $M_{A} \gtrsim 200 \mathrm{GeV}$. This is a reflection of the well known fact that the decoupling limit, in which the $A$ and $H$ states are degenerate in mass and have the same couplings to fermions and vanishing couplings to gauge bosons, is attained very quickly at high $\tan \beta$. Hence, the $h \mathrm{MSSM}$ approach should be a good approximation as it describes correctly this decoupling regime. In turn, at low $\tan \beta$, the mass difference $M_{H}-M_{A}$ can be large and the angle $\alpha$ significantly different from $\beta-\frac{1}{2} \pi$ even for $M_{A} \approx 400 \mathrm{GeV}$ meaning that the decoupling limit is reached slowly in this case. (For $M_{A} \approx M_{h}$ we are close to the regime in which the $h \mathrm{MSSM}$ is not valid and one gets $M_{H} \rightarrow \infty$ and $\alpha-\pi / 2$; this feature will be discussed shortly). 



Figure 3. Contours for the mass difference $M_{H}-M_{A}$ (left) and the coupling squared $\cos ^{2}(\beta-\alpha)$ (right) in the $\left[\tan \beta, M_{A}\right]$ plane. The $h$ mass is fixed to the value $M_{h}=125 \mathrm{GeV}$ and the red area at low $\tan \beta$ and $M_{A}$ is the one where the $h$ MSSM is ill defined.

This statement is made more explicit in figure 3 where contours for the heavier Higgs mass difference $M_{H}-M_{A}$ and the square of the reduced $H$ coupling to massive gauge bosons, $g_{H V V}^{2}=\cos ^{2}(\beta-\alpha)$, which is a very good measure of the distance to the decoupling limit. At $M_{A} \approx 500 \mathrm{GeV}$, the difference $M_{H}-M_{A}$ is less than $1 \mathrm{GeV}$ for $\tan \beta \gtrsim 10$ while it is about $10 \mathrm{GeV}$ for $\tan \beta \approx 2$. However, even in this case, the mass difference represents about $2 \%$ of the $A / H$ masses and, hence in view of the experimental resolution, one can still consider that the two states $A$ and $H$ are degenerate in mass.

There is one problem with the $h \mathrm{MSSM}$ approach at very low $\tan \beta$, though. If $\tan \beta \approx$ 1 , the denominator of eq. (2.2) that expresses the dominant radiative correction $\Delta M_{22}^{2}$ in terms of the measured mass $M_{h}$ becomes close to $M_{Z}^{2}+M_{A}^{2}-2 M_{h}^{2}$ and, at low $M_{A}$, it approaches zero rendering eq. (2.2) ill defined. For $\tan \beta \simeq 1$, the pseudoscalar mass range $M_{A} \lesssim 160 \mathrm{GeV}$ is inaccessible, but the forbidden range shrinks to $M_{A} \lesssim M_{h}$ for $\tan \beta \gtrsim 3$. As a lower bound $M_{A} \gtrsim M_{Z}$ has been set in the model-independent searches of the MSSM Higgs bosons at the LEP2 collider [38], this area in which the hMSSM is not defined is therefore rather small. We will show later that this forbidden area is entirely excluded by the present LHC MSSM Higgs searches, in particular by $H^{ \pm}$and $A$ searches as in both cases, the constraints can be interpreted only in terms of $\tan \beta$ and $M_{A}$ and hence without using the $h$ MSSM relations of eq. (2.6).

In fact, this "theoretically forbidden" hMSSM area is also excluded by the measurement of the observed Higgs boson production and decay rates at the LHC. Indeed, for these low $M_{A}$ and $\tan \beta$ values, we are very far from the decoupling limit in which the couplings of the $h$ boson are close to their SM-like values, as the LHC Higgs data in the various channels seem to strongly indicate [11-13]. However, we will refrain from using this argument to exclude the low $M_{A}, \tan \beta$ possibility, as we will prefer to perform the direct Higgs searches in a model independent manner, without relying on any indirect constraint. 


\section{MSSM Higgs production and decays at the LHC}

We come now to the discussion of the decays and the production at the LHC of the heavier $A, H$ and $H^{ \pm}$particles in the $h$ MSSM. We will be mostly interested in the low $\tan \beta$ region, but we will first summarize the main features at high and moderate $\tan \beta$.

\subsection{Neutral Higgs decays}

At high $\tan \beta$ values, say $\tan \beta \gtrsim 10$, the decay pattern of the heavier neutral $H / A$ bosons is extremely simple $[10,82,83]$ as a result of the strong enhancement of the couplings to down-type quarks and charged leptons that are proportional to $\tan \beta$, not only for the $A$ state but also for the $H$ boson. Indeed, as in the decoupling limit $M_{A} \gg M_{Z}$ on has $\alpha \rightarrow \beta-\frac{1}{2} \pi$, the $H b \bar{b}$ and $H \tau \tau$ couplings normalised to the SM Higgs coupling take the limit

$$
g_{H d d} \equiv \cos \alpha / \cos \beta \stackrel{M_{A} \gg M_{Z}}{\longrightarrow} \tan \beta \equiv g_{\text {Add }} .
$$

The neutral $\Phi=A / H$ states will decay almost exclusively into $\tau^{+} \tau^{-}$and $b \bar{b}$ pairs, with branching ratios of $\operatorname{BR}(\Phi \rightarrow \tau \tau) \approx 10 \%$ and $\operatorname{BR}(\Phi \rightarrow b \bar{b}) \approx 90 \%$. This is a simple consequence of the fact that the partial widths are proportional to respectively $\left(m_{\tau} \tan \beta\right)^{2}$ and $3\left(\bar{m}_{b} \tan \beta\right)^{2}$ when the color factor is included; $m_{\tau}=1.78 \mathrm{GeV}$ and the $\overline{\mathrm{MS}}$ bottom quark mass defined at the scale of the Higgs mass is $\bar{m}_{b} \approx 3 \mathrm{GeV}$, implying thus,

$$
\operatorname{BR}(\Phi \rightarrow \tau \tau) \approx 1-\operatorname{BR}(\Phi \rightarrow b \bar{b}) \approx m_{\tau}^{2} /\left(m_{\tau}^{2}+3 \bar{m}_{b}^{2}\right) \approx 0.1
$$

At high $\tan \beta$, all other decay channels of the $H / A$ states are strongly suppressed. This is particularly the case of the decays into top quark pairs, despite of the large value $m_{t} \gg m_{b}$, as the Higgs coupling to up-type quarks are inversely proportional to $\tan \beta$,

$$
g_{\text {Huи }} \equiv \sin \alpha / \sin \beta \stackrel{M_{A} \gg M_{Z}}{\longrightarrow} 1 / \tan \beta \equiv g_{\text {Auи }}
$$

rendering very small the $\Phi=H, A$ partial widths, given in the Born approximation by

$$
\Gamma(\Phi \rightarrow t \bar{t})=3 G_{F} m_{t}^{2} /(4 \sqrt{2} \pi) \times g_{\Phi t t}^{2} M_{\Phi} \beta_{t}^{p}
$$

where $\beta_{t}=\left(1-4 m_{t}^{2} / M_{\Phi}^{2}\right)^{1 / 2}$ and $p=3$ (1) for the CP-even (CP-odd) Higgs boson.

This is also the case of Higgs decays involving gauge and Higgs particles in the final state. In particular, one should have in principle also the decay modes $H \rightarrow V V$ with $V=W, Z$ and $H \rightarrow h h$ in the case of the CP-even and $A \rightarrow Z h$ in the case of the CP-odd Higgs bosons. However, the partial decay widths of the $H$ particle into massive gauge bosons $\Gamma(H \rightarrow V V)$ are proportional to the square of the reduced coupling

$$
g_{H V V} \equiv \cos (\beta-\alpha) \stackrel{M_{A} \gg M_{Z}}{\longrightarrow} 0 \equiv g_{A V V}
$$

which becomes zero in the decoupling limit as is the case for the pseudoscalar $A$ boson, that has no tree-level couplings to $V V$ states as a result of CP-invariance. For the latter state, the possibility $A \rightarrow h Z$ for $M_{A} \geq M_{h}+M_{Z} \approx 220 \mathrm{GeV}$, i.e. near the decoupling limit, 
will have a suppressed rate as the coupling $g_{A h Z}=g_{H V V}$ tends to zero at large $\tan \beta$. Indeed, an expansion in terms of $1 / M_{A}^{2}$ gives [30]

$$
g_{H V V}=g_{A h Z} \stackrel{M_{A} \gg M_{Z}}{\longrightarrow} M_{Z}^{2} /\left(2 M_{A}^{2}\right) \times \sin 4 \beta-\Delta M_{22}^{2} /\left(2 M_{A}^{2}\right) \times \sin 2 \beta
$$

and, at high $\tan \beta$, both $\sin 4 \beta$ and $\sin 2 \beta$ are proportional to $\cot \beta$ so that the limit $g_{H V V} \rightarrow 0$ is reached faster in this case. The same is true for the decay $H \rightarrow h h$ when $M_{H} \geq 2 M_{h}$ as the trilinear Higgs coupling of eq. (2.10) for $M_{H} \gtrsim 2 M_{h}$ reaches the limit

$$
g_{H h h} \stackrel{M_{A} \gg M_{Z}}{\longrightarrow}-3 \Delta M_{22}^{2} /\left(2 M_{Z}^{2}\right) \times \sin 2 \beta
$$

and is thus strongly suppressed at high $\tan \beta$ that implies $\sin 2 \beta=2 \tan \beta /\left(1+\tan ^{2} \beta\right) \rightarrow 0$.

The situation is drastically different at low values of $\tan \beta$ when the heavy Higgs states are kinematically allowed to decay into top quark pairs, $M_{H} \approx M_{A} \gtrsim 2 m_{t}$ [10]. Indeed, $H / A \rightarrow t \bar{t}$ become by far dominant $g_{\Phi t t} \propto m_{t} / \tan \beta$ is so strong that it leaves no chance to the other possible channels. This is clearly the case for the $H / A \rightarrow b \bar{b}, \tau^{+} \tau^{-}$rates which become negligibly small as the couplings $g_{\Phi d d}$ are not enhanced anymore and $m_{t} \gg m_{b}, m_{\tau}$. This is also the case of the decays $A \rightarrow h Z$ and $H \rightarrow V V$ at large $M_{\Phi}$ since the couplings approach zero in this case. For the $H \rightarrow h h$ decay, there is still a component of the $g_{H h h}$ coupling of eq. (3.7), the one $\alpha \sin 2 \beta \approx 1$ for $\tan \beta \approx 1$, that is non-zero in the decoupling limit. However, besides the fact that the $H t t$ coupling is larger than the $H h h$ coupling, the partial decay width for the process $H \rightarrow h h$ decreases as $1 / M_{H}$

$$
\Gamma(H \rightarrow h h)=\left(G_{F} M_{Z}^{4}\right) /\left(16 \sqrt{2} \pi M_{H}\right) \times \lambda_{H h h}^{2}\left(1-4 M_{h}^{2} / M_{H}^{2}\right)^{1 / 2}
$$

while $\Gamma(H \rightarrow t t) \propto M_{H}$ and, hence, largely dominates at high $M_{H}$ (slightly above $M_{H} \approx 2 m_{t}$ however, $\operatorname{BR}(H \rightarrow h h)$ stays significant as $\Gamma(H \rightarrow t \bar{t})$ is suppressed by $\beta_{t}^{3}$ near threshold).

The situation is opposite when the decays $H \rightarrow V V$ are considered. Indeed, because of the contributions of the longitudinal components of the $V=W, Z$ bosons that grow with the energy scale, the partial decay widths increases as $M_{H}^{3}$ and not as $M_{H}$

$$
\Gamma(H \rightarrow V V)=\frac{G_{F} \delta_{V}}{16 \sqrt{2} \pi} g_{H V V}^{2} M_{\Phi}^{3} \beta_{V}\left(1-\frac{4 M_{V}^{2}}{M_{H}^{2}}+\frac{12 M_{V}^{4}}{M_{H}^{4}}\right) \stackrel{M_{A} \gg M_{Z}}{\longrightarrow} \frac{G_{F} \delta_{V}}{16 \sqrt{2} \pi} g_{H V V}^{2} M_{H}^{3}
$$

where again $\beta_{V}=\left(1-4 M_{V}^{2} / M_{H}^{2}\right)^{1 / 2}$ and $\delta_{V}=2(1)$ for $V=W(Z)$. At low $\tan \beta$ and high $M_{H}$ values, one of the components of $g_{H V V}$ given in eq. (3.6) (the one $\propto \sin 4 \beta$ ) vanishes while the other component tends to $g_{H V V} \rightarrow-\frac{1}{2} \Delta M_{22}^{2} / M_{A}^{2}$. Because of the enhancement of the decay rate by $M_{H}^{3}$, one would have then a partial width $\Gamma(H \rightarrow V V)$ that is suppressed by a power $1 / M_{H}$ only and hence, does not become completely negligible compared to $H \rightarrow t \bar{t}$ even at very high $M_{H}$. For instance, at $M_{H} \approx 500 \mathrm{GeV}$, the branching ratios for the decays $H \rightarrow W W$ and $H \rightarrow Z Z$ are still at the $2 \%$ and $1 \%$ level respectively. This is appreciable and, at least in the case of the $Z Z$ decay, it is of the same order of magnitude as the branching fraction of the observed $125 \mathrm{GeV} h$ boson, with the advantage that the $Z Z$ pair has a much larger invariant mass with a significantly smaller background (which compensates for the smaller Higgs production cross section as will be seen later). 
If the $H / A$ states have masses below the $2 m_{t}$ kinematical threshold, the two-body $H / A \rightarrow \bar{t} t$ decays are not allowed anymore. Off-shell three-body decays $A / H \rightarrow \bar{t} t^{*} \rightarrow \bar{t} b W$ are possible, but the rates are suppressed by an additional electroweak factor and the virtuality of one of the top quarks [84-86]. The gauge and Higgs decays of the $H / A$ states would then become significant at low $\tan \beta$ values. In the mass range $M_{h}+M_{Z} \lesssim M_{A} \lesssim 2 m_{t}$, the decay $A \rightarrow h Z$ will be dominant: the reduced coupling $g_{A h Z}=g_{H V V} \propto M_{h}^{2} / M_{A}^{2}$ is only moderately suppressed and the full $A h Z$ coupling is still substantial compared to the tiny $A b \bar{b}$ coupling. Likewise, for $2 M_{h} \lesssim M_{H} \lesssim 2 m_{t}$, the decay mode $H \rightarrow h h$ is dominant as the coupling $g_{H h h}$ at low $\tan \beta$ will stay appreciable. The two-body decays into massive gauge bosons $H \rightarrow W W$ and $Z Z$ are also significant below the $2 m_{t}$ threshold.

The bosonic decays will also be non-negligible at intermediate values of $\tan \beta, \tan \beta \approx$ $\sqrt{\bar{m}_{t} / \bar{m}_{b}} \approx 5-10$, when the $A / H$ couplings to top quarks are suppressed while those to bottom quarks are not yet strongly enhanced. However, below the $2 m_{t}$ threshold, when the Higgs couplings to the bosonic states are not too suppressed and the only competition will be due to the $\Phi \rightarrow b \bar{b}$ decays that is only slightly enhanced, the rates for the $H \rightarrow$ $W W, Z Z, h h$ and $A \rightarrow Z h$ channels will be smaller than at low $\tan \beta$ values.

The branching fractions for the various Higgs decays discussed above are displayed in figure 4 in the $\left[\tan \beta, M_{A}\right.$ ] plane assuming the $h \mathrm{MSSM}$ with $M_{h}=125 \mathrm{GeV}$. The Fortran program HDECAY $[82,83]$ where the $h$ MSSM relations were implemented has been used. The color code is such that the red area is when the considered decay rates are large, while the blue area is when they are small (for alternative and more easily readable figures for the branching ratios, as well as for the production cross sections, see for instance refs. $[26,30]$ ). The white areas are when the decay rates are very small, below the minimal value of the scale in the color axis. As can be seen, the $H / A \rightarrow \tau \tau$ decays are important at high $\tan \beta$ values. The branching ratios for the decays $H / A \rightarrow b \bar{b}$ follow that of $\tau \tau$ final states when a factor 9 is included and are, hence, largely dominant. The decays $H / A \rightarrow t \bar{t}$ are by far leading at low $\tan \beta$ for $M_{A, H} \gtrsim 350 \mathrm{GeV}$ (one notices that at least for $A$, they are also significant slightly below the $t \bar{t}$ threshold). The bosonic decays $H \rightarrow W W, Z Z, h h$ and also $A \rightarrow h Z$ have reasonable rates only for $\tan \beta \lesssim 3$ and below the $2 m_{t}$ threshold.

A final word should be devoted to the total Higgs decay widths, which are displayed for the $A$ and $H$ particles in figure 5 , again in the $\left[\tan \beta, M_{A}\right] h$ MSSM plane. In the low and high $\tan \beta$ regimes, one can consider only the dominant fermionic decays of the $\Phi$ states and, to a good approximation, the total decay widths read (up to phase space factors)

$$
\Gamma_{H}^{\mathrm{tot}} \approx \Gamma_{A}^{\mathrm{tot}}\left(\approx \Gamma_{H^{ \pm}}^{\mathrm{tot}}\right) \approx G_{F} M_{\Phi} /(4 \sqrt{2} \pi)\left[\left(m_{\tau}^{2}+3 \bar{m}_{b}^{2}\right) \tan ^{2} \beta+3 m_{t}^{2} \cot ^{2} \beta\right] .
$$

For $\tan \beta \approx 1$ and $\tan \beta \approx 60$, one obtains a total decay width that is approximately $\Gamma_{\Phi}^{\text {tot }} \approx 5 \% M_{\Phi}$ and, compared to the Higgs mass, it is not very large. Hence, to a good approximation the $A / H$ states can be considered as narrow resonances in most cases.

Note that for the $H$ state, as the branching ratios and total decay width are shown as a function of $M_{A}$, some peculiar features can be observed. These are explained by the fact that there is a large splitting between $M_{H}$ and $M_{A}$ at low $\tan \beta$ and $M_{A}$ values which lead to, for instance, the opening of the $H \rightarrow t \bar{t}$ mode already at $M_{A} \lesssim 200 \mathrm{GeV}$ and, hence, suppressed $H \rightarrow W W, Z Z$ decays but a large total decay width for $\tan \beta \approx 1$. 
$\mathrm{BR}(\mathrm{A} \rightarrow \tau \tau)$

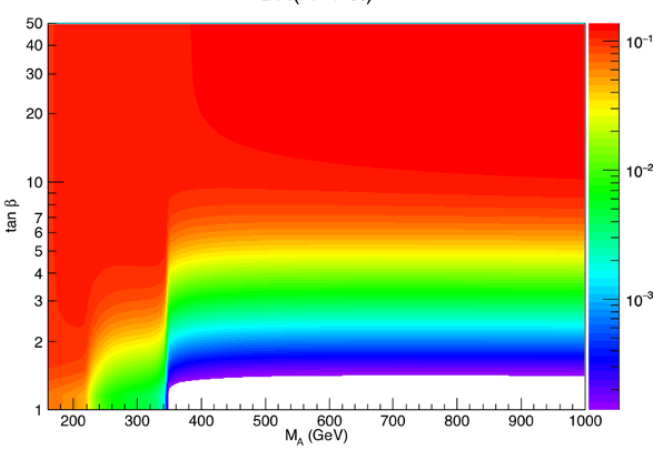

$\mathrm{BR}(\mathrm{A} \rightarrow \mathrm{hZ})$

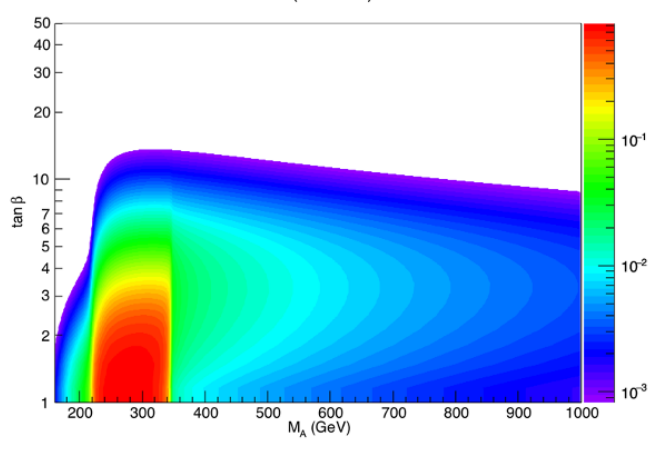

$\mathrm{BR}\left(\mathrm{H} \rightarrow \mathrm{W}^{+} \mathrm{W}\right)$
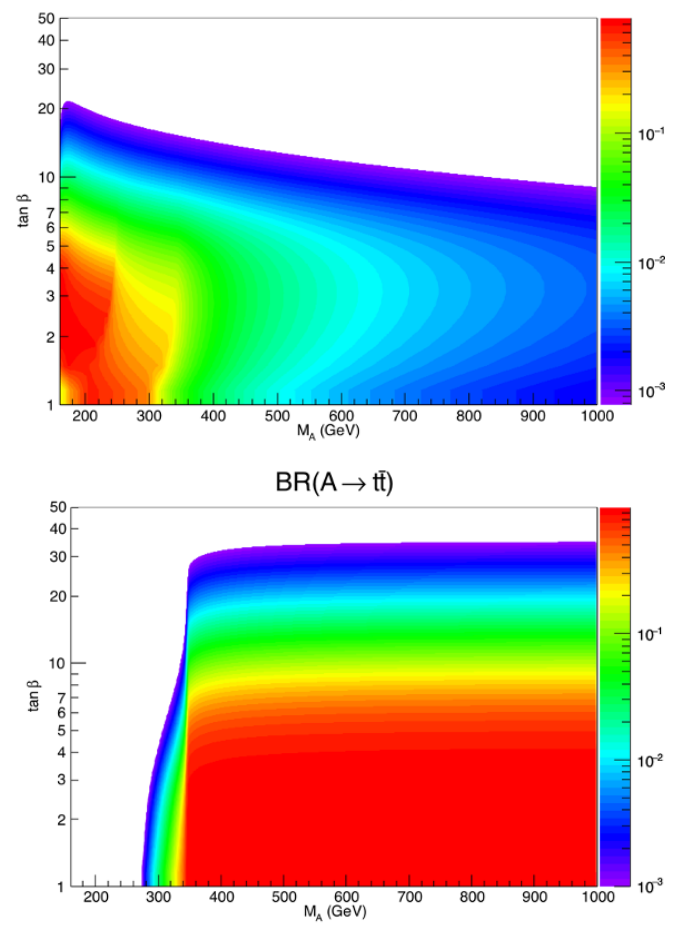

$\mathrm{BR}(\mathrm{H} \rightarrow \tau \tau)$

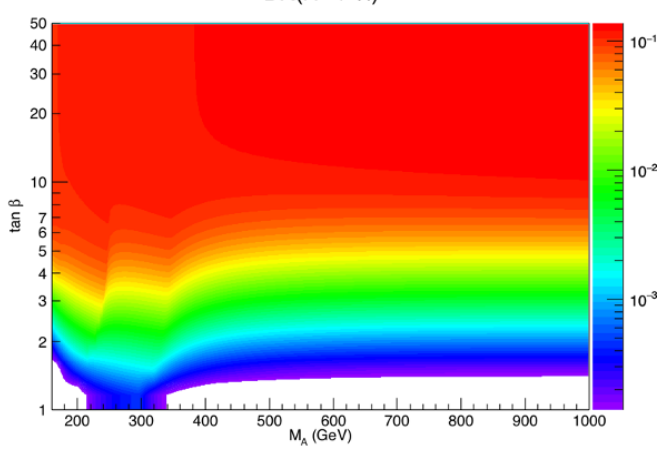

$\mathrm{BR}(\mathrm{H} \rightarrow \mathrm{hh})$

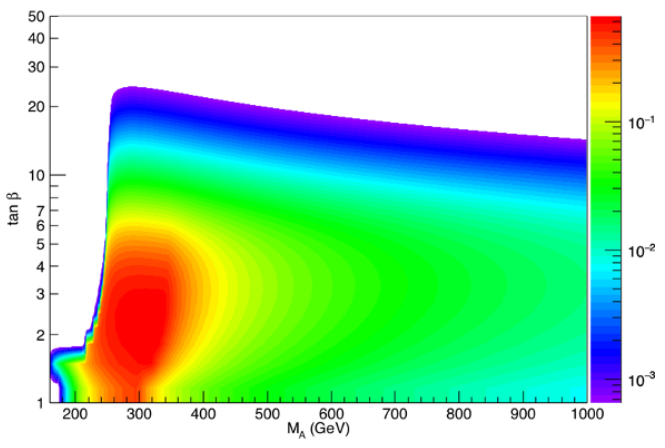

$\mathrm{BR}(\mathrm{H} \rightarrow \mathrm{ZZ})$
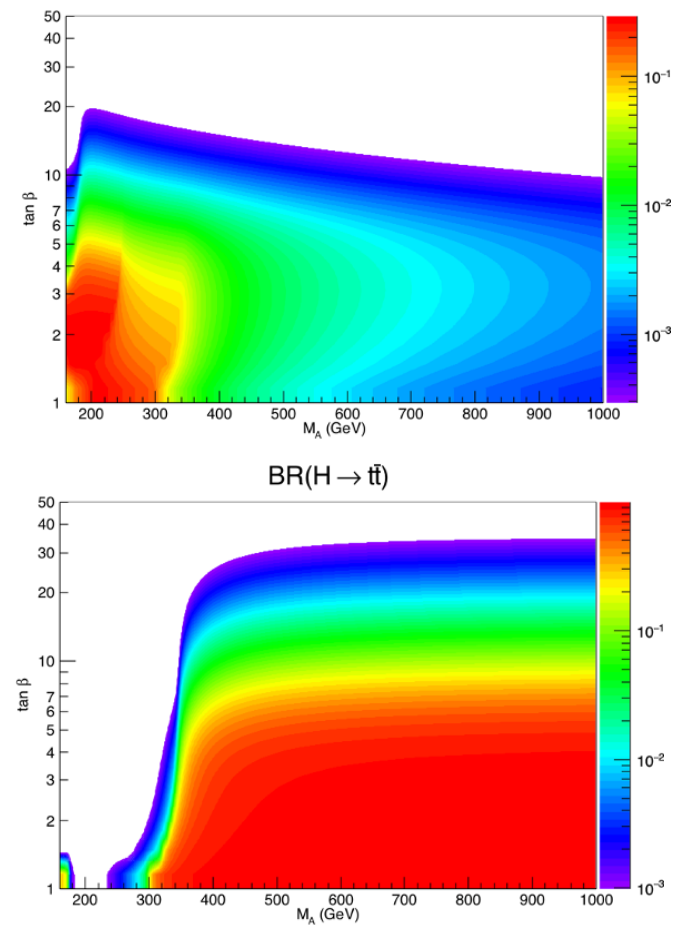

Figure 4. The branching ratios of the neutral Higgs bosons in the $\left[\tan \beta, M_{A}\right]$ parameter space of the $h$ MSSM with the constraint $M_{h}=125 \mathrm{GeV}$. Shown are the rates for the decays $A / H \rightarrow \tau \tau$ (top), $A \rightarrow h Z$ and $H \rightarrow h h, W W, Z Z$ (middle) and $A / H \rightarrow t \bar{t}$ (bottom). 
$\Gamma(\mathrm{A})(\mathrm{GeV})$

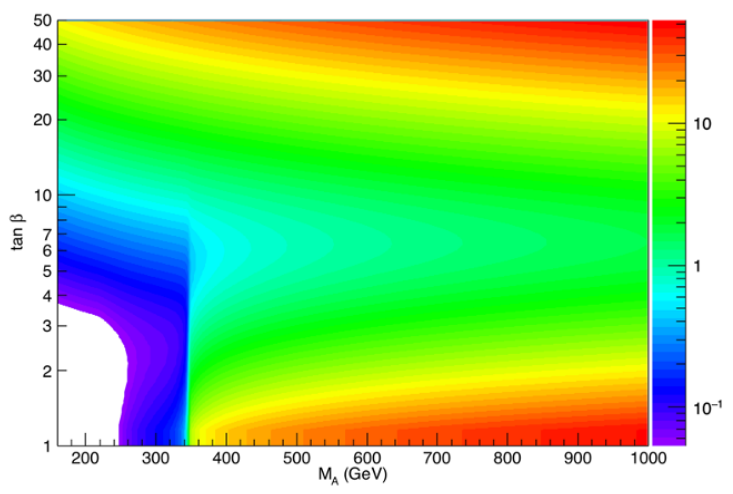

$\Gamma(\mathrm{H})(\mathrm{GeV})$

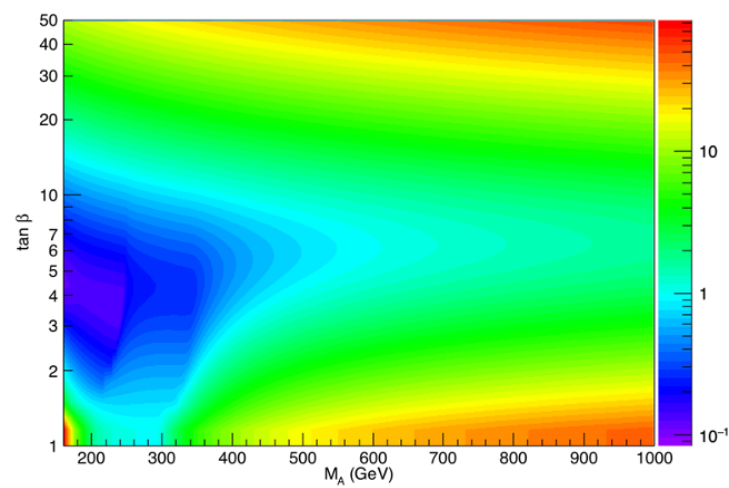

Figure 5. The total decay widths of the neutral $A$ (left) and $H$ (right) bosons in the $\left[\tan \beta, M_{A}\right]$ parameter space of the $h \mathrm{MSSM}$ with the constraint $M_{h}=125 \mathrm{GeV}$.

\subsection{Neutral Higgs production}

Let us turn now to the production of the neutral MSSM $\Phi=H / A$ bosons at the LHC. Also in this case, the cross sections crucially depend on the considered $\tan \beta$ regime and in most cases, the two processes that play a leading role are the gluon-fusion mechanism $g g \rightarrow \Phi$ which is initiated by a heavy quark loop [87] and the associated Higgs production with $b$-quarks, $g g / q \bar{q} \rightarrow b \bar{b}+\Phi$, which at high energies and if no-additional $b$-quark is considered in the final state, is equivalent to the fusion process $b \bar{b} \rightarrow \Phi$ [88-90]. All other processes, in particular vector boson fusion and associated production with a massive gauge boson for the CP-even $H$ state, $q q \rightarrow H q q$ and $q \bar{q} \rightarrow H V$, and associated production with top-quark pairs for both the $H$ and $A$ states, $p p \rightarrow \Phi t \bar{t}$, have much smaller rates as the couplings $g_{H V V}$ and $g_{\Phi t t}$ are suppressed and/or the available phase space is not favorable.

At leading order in perturbation theory, the partonic cross sections for the $b \bar{b} \rightarrow \Phi$ and $g g \rightarrow \Phi$ processes can be written in terms of the partonic c.m. energy $\hat{s}$ and $M_{\Phi}$ as [6-8]

$$
\begin{aligned}
\hat{\sigma}(b \bar{b} \rightarrow \Phi) & =\frac{\pi}{12} g_{\Phi b b}^{2} \delta\left(\hat{s}-M_{\Phi}^{2}\right) \\
\hat{\sigma}(g g \rightarrow \Phi) & =\frac{G_{F} \alpha_{s}^{2}}{288 \sqrt{2} \pi} M_{\Phi}^{2} \delta\left(\hat{s}-M_{\Phi}^{2}\right)\left|\frac{3}{4} \sum_{Q} g_{\Phi Q Q} A_{1 / 2}^{\Phi}\left(\tau_{Q}\right)\right|^{2} .
\end{aligned}
$$

In the case of the $g g \rightarrow \Phi$ process, the quarks $Q$ running in the loop should be taken to be the heavy bottom and top quarks with Higgs couplings given in eq. (2.9) and masses incorporated into the reduced variables $\tau_{Q}=M_{\Phi}^{2} / 4 m_{Q}^{2}$. The form factors for spin- $\frac{1}{2}$ fermion loops in the case of a $\mathrm{CP}$-even $H$ and a $\mathrm{CP}$-odd $A$ bosons are given by

$$
\begin{aligned}
& A_{1 / 2}^{H}(\tau)=2[\tau+(\tau-1) f(\tau)] \tau^{-2} \\
& A_{1 / 2}^{A}(\tau)=2 \tau^{-1} f(\tau)
\end{aligned}
$$


where the function $f(\tau)$ above and below the $\tau=1$ kinematical threshold is defined as

$$
f(\tau)= \begin{cases}\arcsin ^{2} \sqrt{\tau} & \tau \leq 1 \\ -\frac{1}{4}\left[\log \frac{1+\sqrt{1-\tau^{-1}}}{1-\sqrt{1-\tau^{-1}}}-i \pi\right]^{2} & \tau>1 .\end{cases}
$$

While the amplitudes are real for $M_{\Phi} \leq 2 m_{Q}$, they develop an imaginary part above the kinematical threshold. At very low Higgs masses, compared to the internal quark mass, the amplitudes for a scalar and a pseudoscalar states reach constant but different values

$$
M_{\Phi} \ll 2 m_{Q}: A_{1 / 2}^{H}\left(\tau_{Q}\right) \rightarrow 4 / 3, A_{1 / 2}^{A}\left(\tau_{Q}\right) \rightarrow 2 .
$$

Instead, in the opposite limit, $M_{\Phi} \gg 2 m_{Q}$, chiral symmetry holds and the amplitudes for the CP-even and a CP-odd Higgs bosons are identical (as in the $b \bar{b} \rightarrow \Phi$ case),

$$
M_{\Phi} \gg 2 m_{Q}: A_{1 / 2}^{\Phi}\left(\tau_{Q}\right) \rightarrow-\left[\log \left(4 \tau_{Q}\right)-i \pi\right]^{2} /\left(2 \tau_{Q}\right) .
$$

The maximal values of the amplitudes occur slightly above the kinematical threshold where one has for the real parts $\operatorname{Re}\left(A_{1 / 2}^{H}\right) \sim 2$ and $\operatorname{Re}\left(A_{1 / 2}^{A}\right) \sim 5$.

At high $\tan \beta$, the strong enhancement of the Higgs couplings to $b$-quarks and the suppression of the couplings to top quarks and gauge massive bosons makes that only these two processes are relevant, with the gluon-gluon fusion mechanisms dominantly generated by the bottom quark loop. The cross sections $\sigma(g g \rightarrow \Phi)$ and $\sigma(b \bar{b} \rightarrow \Phi)$ are of the same order of magnitude and can be so large that they make the process $p p \rightarrow g g+b \bar{b} \rightarrow \Phi \rightarrow \tau^{+} \tau^{-}$, with the branching fraction for the decay $\Phi \rightarrow \tau^{+} \tau^{-}$being of order $10 \%$ as seen previously, the most powerful LHC search channel for the heavier MSSM Higgs bosons. The $p p \rightarrow b \bar{b}+H / A$ mode with $A / H \rightarrow b \bar{b}$, which has an order of magnitude larger rates in principle (if no high- $p_{T} b$-quark from production is required), has also been considered but the sensitivity is smaller as this fully hadronic process is subject to a much larger QCD background.

At high $\tan \beta$, the production rates are approximately the same for the $H$ and $A$ states in both the $b \bar{b}$ and $g g$ fusion cases as discussed earlier. While $\sigma(b b \rightarrow \Phi)$ is known up to NNLO in QCD perturbative theory [91], $\sigma(g g \rightarrow \Phi)$ is instead known only up to NLO in the limit $M_{\Phi} \gtrsim 2 m_{Q}$ that we will be mainly interested in [92]. For the top-quark loop, we will nevertheless also include the NNLO QCD corrections [93-96] that are in principle only valid for $M_{\Phi} \lesssim 2 m_{Q}$ as advocated in ref. [97]. The precise values of the cross section times branching fractions $\sigma(p p \rightarrow \Phi) \times \mathrm{BR}\left(\Phi \rightarrow \tau^{+} \tau^{-}\right)$for a given $\left[\tan \beta, M_{A}\right.$ ] MSSM point have been updated in refs. [97-100] and the associated theoretical uncertainties from missing higher order perturbative contributions, the parametrisation of the parton distribution functions and uncertainties on the inputs $\alpha_{s}$ and $b$-quark mass, have been estimated to be of the order of 25\%. Any effect below this level, such as the SUSY effects that we will be discussed later in this section, should be considered as small.

Again, at low $\tan \beta$, the situation is very different. The cross sections for the $b \bar{b} \rightarrow \Phi$ processes are now very small as the $\Phi b \bar{b}$ coupling is not enhanced anymore. For $M_{A} \gtrsim 200 \mathrm{GeV}$, this is also the case of the associated production with $t \bar{t}$ pairs as a result of a the small phase-space and, in the case of the $H$ state, of the vector boson fusion 
$q q \rightarrow H q q$ and associated production with a gauge boson $q \bar{q} \rightarrow H V$ as a result of the suppressed $H V V$ coupling (the $A$ state cannot be produced in these two processes as there is no $A V V$ coupling). The only process which would have a reasonable production cross section would be the gluon-fusion process $g g \rightarrow \Phi$ with, this time, the leading contribution being generated by loops of top quarks that have couplings to the Higgs bosons that are only slightly suppressed compared to the SM Higgs case.

In figure 6, the production cross sections $\sigma(g g \rightarrow \Phi+X)$ and $\sigma(b \bar{b} \rightarrow \Phi+X)$ with $\Phi=A$ (left) and $\Phi=H$ (right) are displayed at the LHC in the $\left[\tan \beta, M_{A}\right] h$ MSSM parameter space for $\sqrt{s}=8 \mathrm{TeV}$ (top) and $14 \mathrm{TeV}$ (bottom); the MSTW parton distribution functions [101] have been used. The rates for $b \bar{b} \rightarrow \Phi$ have been obtained using the program SusHi [102] and an adapted version of HIGLU [103, 104] has been used for $g g \rightarrow \Phi$. As can be seen, the cross sections are rather large in particular at high $\tan \beta$ and, for $g g \rightarrow \Phi$, also at low $\tan \beta$ when the Higgs couplings to $b$ - or $t$-quarks are strong and at relatively low $M_{A}$ when the phase space is not too penalizing. Even for $M_{A}=500 \mathrm{GeV}(1 \mathrm{TeV})$, the production rates are significant at $\sqrt{s}=8 \mathrm{TeV}(14 \mathrm{TeV})$, if $\tan \beta$ is sufficiently high or low.

\subsection{The case of the charged Higgs boson}

A final word should be devoted to the case of the charged Higgs boson, whose coupling to fermions is proportional to

$$
g_{H^{ \pm} \bar{u} d} \propto m_{d} \tan \beta\left(1+\gamma_{5}\right)+m_{u} \cot \beta\left(1-\gamma_{5}\right) .
$$

The coupling is large at low $\tan \beta$ when the component $m_{u} / \tan \beta$ is not suppressed and at very large $\tan \beta$ when the component $m_{d} \tan \beta$ is enhanced, so that many aspects discussed for the pseudoscalar Higgs boson hold also in this case [10]. At low mass, $M_{H^{ \pm}} \lesssim 160 \mathrm{GeV}$, which corresponds to $M_{A} \lesssim 140 \mathrm{GeV}$, the charged Higgs boson can be produced in the decay of top quarks that are copiously produced at the LHC, $g g+q \bar{q} \rightarrow t \bar{t}$ with one top quark decaying into the dominant $t \rightarrow b W$ mode and the other into $t \rightarrow b H^{+}$. For $M_{H^{ \pm}} \approx 140 \mathrm{GeV}$, the latter channel has a branching ratio ranging from order $\approx 10 \%$ for $\tan \beta \approx 1$ or $\tan \beta \approx 60$ to order $\approx 1 \%$ for $\tan \beta \approx 7-8$ when the Higgs couplings are the smallest. In this low mass range above, the $H^{ \pm}$boson will decay almost exclusively into $\tau \nu$ final states but some some competition with the hadronic decay channel $H^{+} \rightarrow c \bar{s}$ will occur at very low $\tan \beta$. The $H^{ \pm}$branching fractions are shown in figure 7 as a function of $M_{H^{ \pm}}$for two representative $\tan \beta$ values, $\tan \beta=2$ and $\tan \beta=30$.

At higher masses, the $H^{ \pm}$state will be mainly produced in the three-body production process $p p \rightarrow t b H^{ \pm}$which, at high energies, is equivalent to the two-body channel $g b \rightarrow$ $H^{ \pm} t$ if no additional final state $b$ quarks are detected [105-107]. Again, significant rates occur only at very low or very large values of $\tan \beta$ when the $H^{ \pm} t b$ coupling of eq. (3.17) is large (some small additional contributions from the tree-level $q \bar{q} \rightarrow H^{+} H^{-}$and loop induced $g g \rightarrow H^{+} H^{-}$pair and associated $q \bar{q} \rightarrow H^{ \pm}+A / h / H$ production modes are also possible [10]). The cross sections have been derived in ref. [108] where the two possibilities for the process, $p p \rightarrow t \bar{b} H^{-}$and $g b \rightarrow t H^{-}$, are properly matched and some numerical grids have been provided for the MSSM. The output of these grids for the production rates at $\sqrt{s}=8$ and $14 \mathrm{TeV}$ is shown in figure 8 in the $\left[\tan \beta, M_{H^{ \pm}}\right]$plane. 

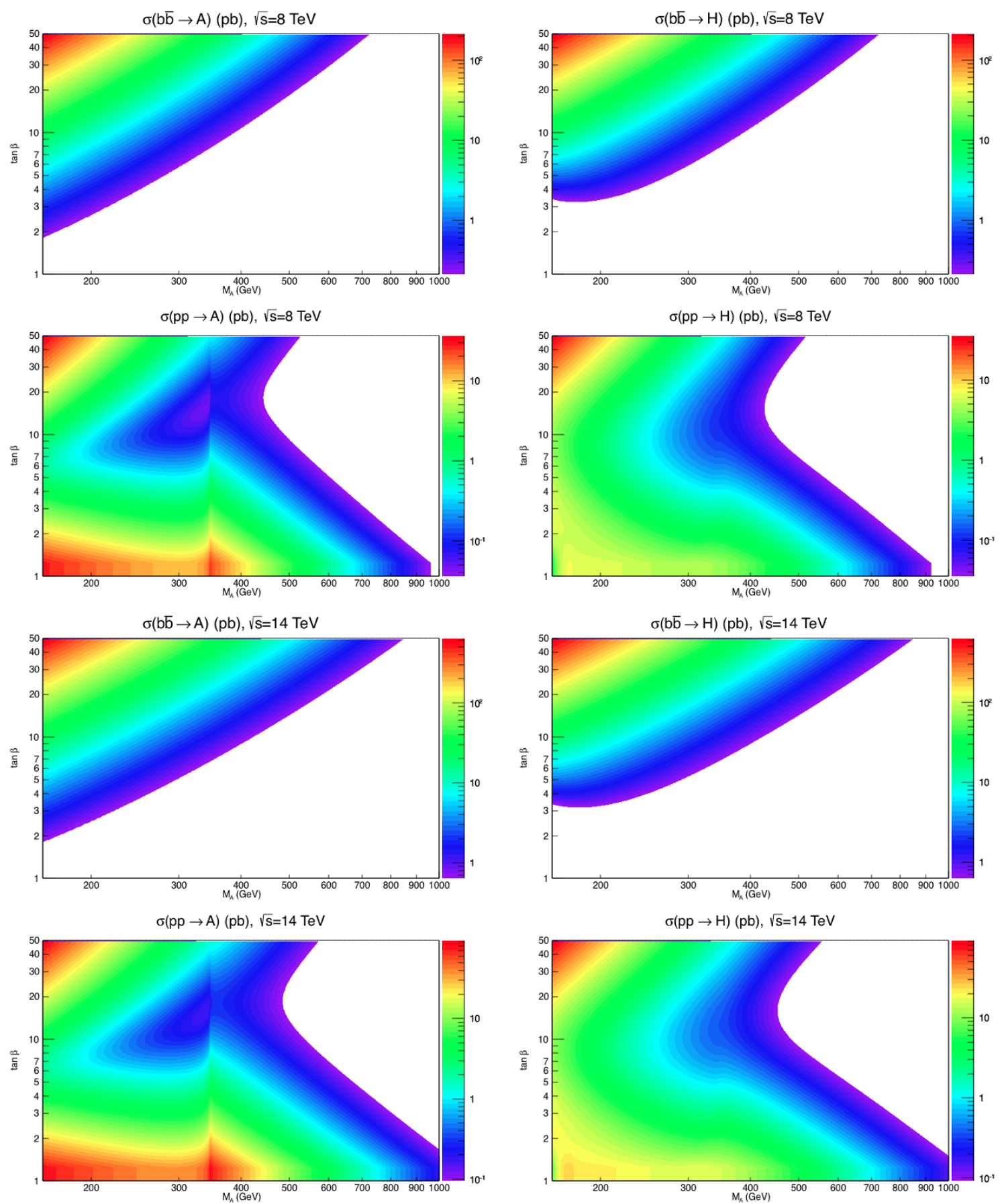

Figure 6. The production cross sections of the MSSM heavier Higgs bosons $A$ (left) and $H$ (right) at the LHC with $\sqrt{s}=8 \mathrm{TeV}$ (top) and $14 \mathrm{TeV}$ (bottom) in the [tan $\left.\beta, M_{A}\right] h \mathrm{MSSM}$ plane; the channels $g g \rightarrow H / A$ and $b \bar{b} \rightarrow H / A$ have been considered.

At high $\tan \beta$, as shown in figure 7 , the $H^{ \pm}$decay branching fractions are $\mathrm{BR}\left(H^{+} \rightarrow\right.$ $\tau \nu) \approx 10 \%$ and $\operatorname{BR}\left(H^{+} \rightarrow t \bar{b}\right) \approx 90 \%$ exactly for the same reasons discussed previously for the $H / A$ particles. All the other decay channels can be safely ignored so that the 

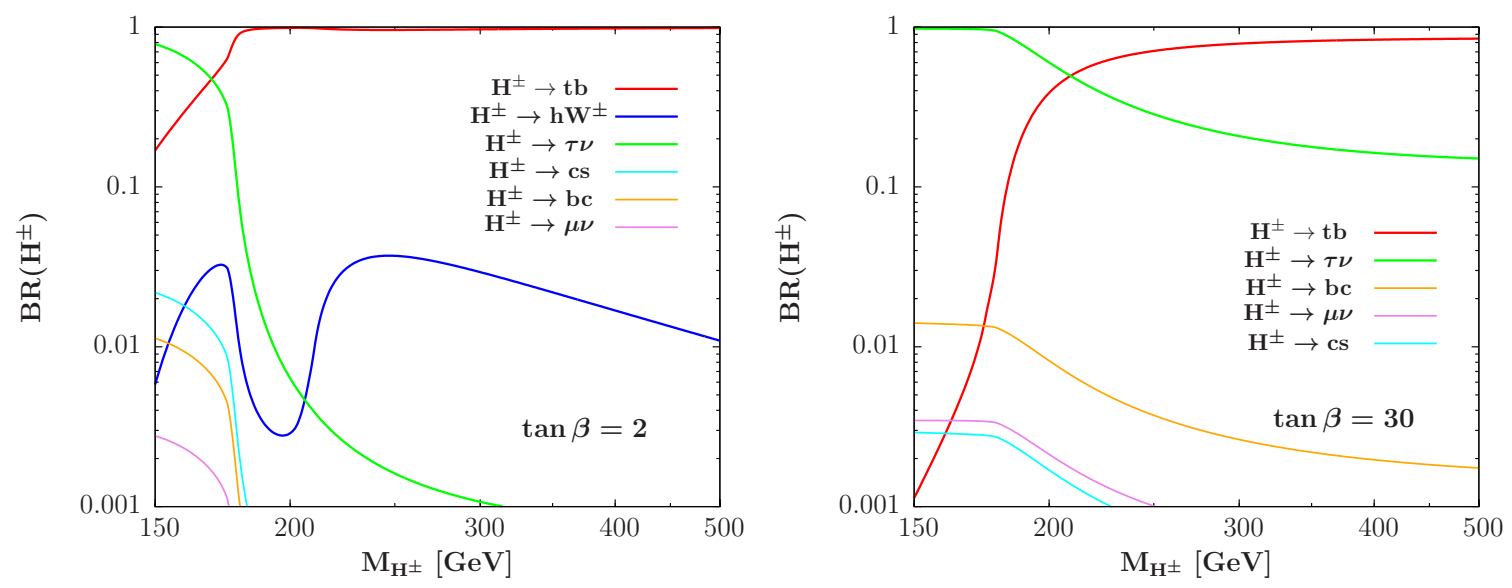

Figure 7. The branching ratios of the charged Higgs boson as a function of its mass for $\tan \beta=2$ (left) and $\tan \beta=30$ (right); the $h \mathrm{MSSM}$ with the constraint $M_{h}=125 \mathrm{GeV}$ is assumed.
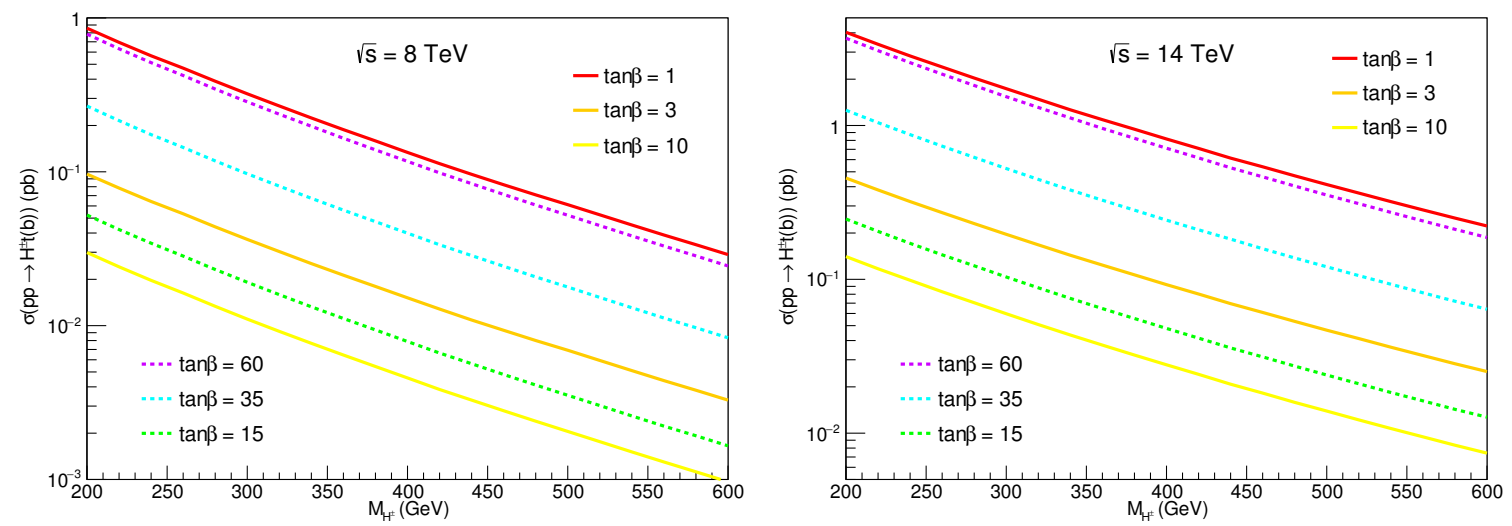

Figure 8. The matched production cross sections $p p \rightarrow H^{+} t(b)$ at the LHC with $\sqrt{s}=8 \mathrm{TeV}$ (left) and $14 \mathrm{TeV}$ (right) as a function of $M_{H^{ \pm}}$for several $\tan \beta$ values; the numbers were taken from the grids given in ref. [108].

main search channel would be $p p \rightarrow H^{ \pm} t(b)$ production with $H^{+} \rightarrow \tau \nu$. As will be seen later, the process is however less powerful in probing the MSSM parameter space than the $p p \rightarrow H / A \rightarrow \tau \tau$ channel discussed earlier.

At low $\tan \beta$, the dominant decay channel will be by far the $H^{+} \rightarrow t \bar{b}$ mode with a branching ratio close to unity for a sufficiently heavy $H^{ \pm}$state when phase-space effects are irrelevant (one should note though, that slightly below the $m_{t}+m_{b}$ kinematical threshold, the three-body decay with an off-shell top quark, $H^{+} \rightarrow b t^{*} \rightarrow b \bar{b} W$, is also important in the case $\tan \beta \approx 2$ as shown in figure 7 ). The main search channel in this case would be the $p p \rightarrow H^{ \pm} t(b) \rightarrow t t b b$ mode. This process, which is sensitive to the same area of $\left[\tan \beta, M_{A}\right]$ parameter space as the $H / A \rightarrow t \bar{t}$ channels discussed before i.e. the low $\tan \beta$ and high $M_{A}$ regions, has been considered in the past and found to be of limited use as it is subject to a large QCD background [109]. However, a recent CMS analysis [110] gave interesting and more optimistic results that we will discuss in the next section. 
Finally, at $H^{ \pm}$masses above $M_{H^{ \pm}} \gtrsim 160 \mathrm{GeV}$, an interesting decay channel would occur, namely $H^{ \pm} \rightarrow W h$. Nevertheless, and in contrast to the similar $A \rightarrow h Z$ decay mode discussed previously, this channel has to compete from the beginning with the dominant $H^{ \pm} \rightarrow t b$ decay. Only at moderate $\tan \beta$ and low $M_{H^{ \pm}}$that this decay has a sizeable branching ratios, of the order of a few percent, allowing for $H^{ \pm}$searches in the interesting channel $p p \rightarrow t b H^{ \pm} \rightarrow t b W h$ which, experimentally, has not been considered so far.

\subsection{Impact of the SUSY spectrum and dark matter}

An important question would be if the MSSM Higgs production times decay rates are not affected by the presence of supersymmetric particles. These could have two impacts: first, they could contribute virtually to the processes and modify the production cross sections and decay branching ratios. This issue is directly related to the third assumption of the $h \mathrm{MSSM}$, namely that the couplings of the Higgs bosons are simply given by eqs. (2.9)(2.10) and no direct correction is involved. Second, SUSY particles could appear in the decays of the Higgs particles and alter the branching ratios for the standard channels that are searched for. This possibility would also invalidate the simple $h$ MSSM approach as some SUSY parameters would be then required to describe Higgs phenomenology. Both issues have been discussed e.g. in ref. [30] and below, we simply summarise the main points with details concerning the low $\tan \beta$ region and the decays $H / A \rightarrow t \bar{t}$.

For what concerns the production processes, besides the standard top and bottomquark loops, there are also squark (and mainly stop) loops [111-115] that contribute to the production of the CP-even $H$ boson in the gluon-fusion channel, $g g \rightarrow H$; the CP-odd $A$ states does not couple to identical sfermions and there is no-squark contribution to $g g \rightarrow A$ at lowest order. However, as the Higgs-squark couplings are not proportional to squark masses, the contributions are damped by powers of the squark mass squared $\propto 1 / \tilde{m}_{Q}^{2}$ and should be small for sufficiently heavy squarks. This is particularly the case at high $\tan \beta$ values where the standard bottom-quark contributions are so strongly enhanced that the impact of squarks becomes negligible. At low $\tan \beta$ values, as one needs a large SUSY scale $M_{S} \gg 1 \mathrm{TeV}$ in order to accommodate an $h$ boson with a mass $M_{h} \approx 125 \mathrm{GeV}$, the impact of the too heavy squarks should also be negligible in the gluon-fusion process. Hence, in most cases, these SUSY loop contributions can be ignored in the production modes.

SUSY particles can have a large impact also through the Higgs boson couplings. Indeed, besides the radiative corrections that affect the Higgs mass matrix eq. (2.1), there are additional one-loop vertex corrections that modify the Higgs-fermion couplings and which are not described by the $h$ MSSM. These corrections are in general only important in the case of $b$-quarks and only at high-tan $\beta$ and large $\mu$ values, since they grow as $\mu \tan \beta$. The dominant components are due to the contributions to the Higgs- $b \bar{b}$ vertices from the strongly interacting sbottoms and gluinos and the weakly interacting higgsinos with top squarks. They can be approximated by $[116,117]$

$$
\Delta_{b} \simeq\left[\frac{2 \alpha_{s}}{3 \pi} m_{\tilde{g}} / \max \left(m_{\tilde{g}}^{2}, m_{\tilde{b}_{i}}^{2}\right)+\frac{\lambda_{t}^{2}}{16 \pi^{2}} A_{t} / \max \left(\mu^{2}, m_{\tilde{t}_{i}}^{2}\right)\right] \mu \tan \beta
$$


They affect mainly the heavier Higgs couplings that become in the limit $M_{A} \gg M_{Z}$,

$$
g_{H b b} \approx g_{A b b} \approx g_{H^{ \pm} t b} \approx \frac{\tan \beta}{1+\Delta_{b}} \text { at high } \tan \beta .
$$

For the lighter $h$ state, the coupling $g_{h b b}$ is not affected in this limit and stays SM-like.

Nevertheless, as already discussed in many places including refs. [30, 98-100], this correction has only a limited impact in the case of the full $p p \rightarrow \Phi=H / A \rightarrow \tau \tau$ process as the correction appears in both the production cross section

$$
\sigma(g g+b \bar{b} \rightarrow \Phi) \propto\left(1+\Delta_{b}\right)^{-2}
$$

and in the $\tau \tau$ decay branching fraction,

$$
\operatorname{BR}(\Phi \rightarrow \tau \tau)=\Gamma(\Phi \rightarrow \tau \tau) /\left[\left(1+\Delta_{b}\right)^{-2} \Gamma(\Phi \rightarrow b \bar{b})+\Gamma(\Phi \rightarrow \tau \tau)\right]
$$

and it largely cancels out in the product of the two

$$
\sigma \times \mathrm{BR} \simeq 1-\frac{1}{5} \Delta_{b}
$$

Hence, only when the $\Delta_{b}$ correction is huge and larger than unity (a feature that might put in danger the perturbative series) that its impact on the $p p \rightarrow \tau \tau$ cross section times decay rate is of the order of the theoretical uncertainty, about $25 \%$ as discussed earlier. At low $\tan \beta$ values, and eventually also at intermediate $\tan \beta$ values, the $\Delta_{b}$ correction is not enhanced and its effects should be rather small. Nevertheless, this is not the case of all process and, in particular, the search channel $p p \rightarrow H / A$ with $H / A \rightarrow b \bar{b}$ in which the $\Delta_{b}$ impact is in fact doubled in the production times decay rates, and the $p p \rightarrow H^{ \pm} b t$ mode with $H^{+} \rightarrow \tau \nu$ at low $M_{H^{ \pm}}$and $H^{+} \rightarrow t b$ at high $M_{H^{ \pm}}$. We will see, however, that these processes do not play a leading role in MSSM Higgs searches at the LHC at high $\tan \beta$. In summary, and to first approximation, one can thus consider that the $\Delta_{b}$ correction has a limited impact on the Higgs searches in the $h$ MSSM.

For the second option, namely that light SUSY particles could contribute to the decays of the Higgs bosons, the situation is also relatively simple; see ref. [10] for a review. At very high $\tan \beta$, the partial widths of the $H / A \rightarrow b \bar{b}, \tau^{+} \tau^{-}$as well as $H^{+} \rightarrow t \bar{b}, \tau^{+} \nu$ decay modes are so strongly enhanced, that they leave no room for the SUSY decay channels.

At low $\tan \beta$, high values of the SUSY scale are required, resulting in large squark masses (at least in universal models in which the squark masses of the three generations are related) that make the Higgs decays into squarks kinematically closed for reasonable $M_{A}$ values. If the masses of the sleptons are disconnected from the SUSY scale and are made small enough for the decays of the heavier Higgs bosons into slepton pairs, $H \rightarrow \tilde{\ell}_{i} \tilde{\ell}_{j}$ and $A \rightarrow \tilde{\ell}_{1} \tilde{\ell}_{2}$ (again, CP invariance forces the $A$ boson not to couple to identical sfermions), to occur. Nevertheless, except for the Higgs-stau couplings at sufficiently high $\tan \beta$ values when the competition from the standard channels is though, the Higgs-sleptons couplings are in general small making these channels very rare and their impact limited. 
Thus, only decays into charginos and neutralinos could play a role and affect significantly the Higgs branching fractions in the standard channels. ${ }^{5}$ Let us briefly comment on these channels.

Three conditions must be fulfilled in order to have significant rates for Higgs decays into charginos and neutralinos, $H / A \rightarrow \chi_{i}^{0} \chi_{j}^{0}$ (with $i, j=1 \cdots 4$ ), $H / A \rightarrow \chi_{i}^{ \pm} \chi_{j}^{\mp}$ (with $i, j=1,2)$ and $H^{ \pm} \rightarrow \chi_{i}^{ \pm} \chi_{j}^{0}[10,119-122]$.

i) One needs that some of the $\chi$ states are light, $M_{\Phi} \gtrsim 2 m_{\chi}$, in order to allow for some decay channels to be kinematically open.

ii) One needs to have significant $\Phi \chi \chi$ couplings; these couplings are maximal when the $\chi$ final states are mixtures of higgsinos and gauginos, a feature which requires comparable higgsino and gaugino mass parameters, $\mu \approx M_{2}$.

iii) One needs that the standard Higgs decay modes are not enhanced and hence, not too low or too large values of $\tan \beta$ where, respectively, the Higgs-top and the Higgsbottom couplings are enhanced.

The maximal Higgs decay rates into charginos and neutralinos are obtained at moderate $\tan \beta$ when all $\chi \chi$ channels are kinematically accessible. In this case, as a consequence of the unitarity of the diagonalizing chargino and neutralino mixing matrices, the sum of the partial widths do not involve any of the elements of these matrices in the asymptotic regime $M_{\Phi} \gg 2 m_{\chi}$ where phase space effects can be neglected. The sum of the branching fractions of the three Higgs bosons $\Phi=H / A / H^{ \pm}$decaying into the various $\chi \chi$ final states is then simply given by ( $\theta_{W}$ is the electroweak mixing angle) [119-122]

$$
\operatorname{BR}\left(\Phi \rightarrow \sum_{i, j} \chi_{i} \chi_{j}\right)=\frac{\left(1+\frac{1}{3} \tan ^{2} \theta_{W}\right) M_{W}^{2}}{\left(1+\frac{1}{3} \tan ^{2} \theta_{W}\right) M_{W}^{2}+\bar{m}_{t}^{2} \cot ^{2} \beta+\left(\bar{m}_{b}^{2}+m_{\tau}^{2}\right) \tan ^{2} \beta}
$$

when only the leading $t \bar{t}, b \bar{b}$ and $\tau \tau$ modes for the neutral and the $t \bar{b}$ and $\tau \nu$ modes for the charged Higgs bosons are included in the total widths. This is approximately the case as we are close to the decoupling limit when these SUSY channels are accessible and the other standard decay modes such as $H \rightarrow V V, h h$ and $A \rightarrow h Z$ are suppressed.

The branching ratios when all ino states are summed up are shown for the three MSSM Higgs states in figure 9 as a function of $\tan \beta$ for $M_{A}=600 \mathrm{GeV}$. The $h \mathrm{MSSM}$ relations for the Higgs sector have been enforced and the other relevant SUSY parameters are fixed to $\mu=M_{2}=200 \mathrm{GeV}$, assuming that the bino and wino soft SUSY-breaking parameters are related by the unification condition $M_{2} \approx 2 M_{1}$. One can see that the branching ratios for

\footnotetext{
${ }^{5}$ Here we will consider models in which the neutralino $\chi_{1}^{0}$ is the lightest SUSY particle (LSP). In gauge mediated SUSY breaking models (GMSB), a very light gravitino can be the LSP and decays of the heavier Higgs bosons into a gravitino and a neutralino or a chargino, $H / A \rightarrow \tilde{G} \chi_{i}^{0}$ and $H^{ \pm} \rightarrow \tilde{G} \chi_{i}^{ \pm}$, are possible (Higgs bosons do not couple to pairs of gravitinos). However, as discussed in ref. [118], the partial widths for these decays are inversely proportional to the square of the SUSY breaking scale, $M_{S}^{2}=\left(m_{\tilde{G}} M_{\mathrm{Planck}}\right)^{2}$, and need $M_{S} \lesssim \mathcal{O}$ (few $100 \mathrm{GeV}$ ) to be substantial. This possibility is ruled out not only from direct SUSY particle searches but also from the fact that the large value of the MSSM $h$ boson mass, $M_{h}=125 \mathrm{GeV}$, requires a SUSY scale in the multi TeV range in GMSB scenarios ( $M_{S} \gtrsim 3 \mathrm{TeV}$ in minima GMSB) [70-79].
} 


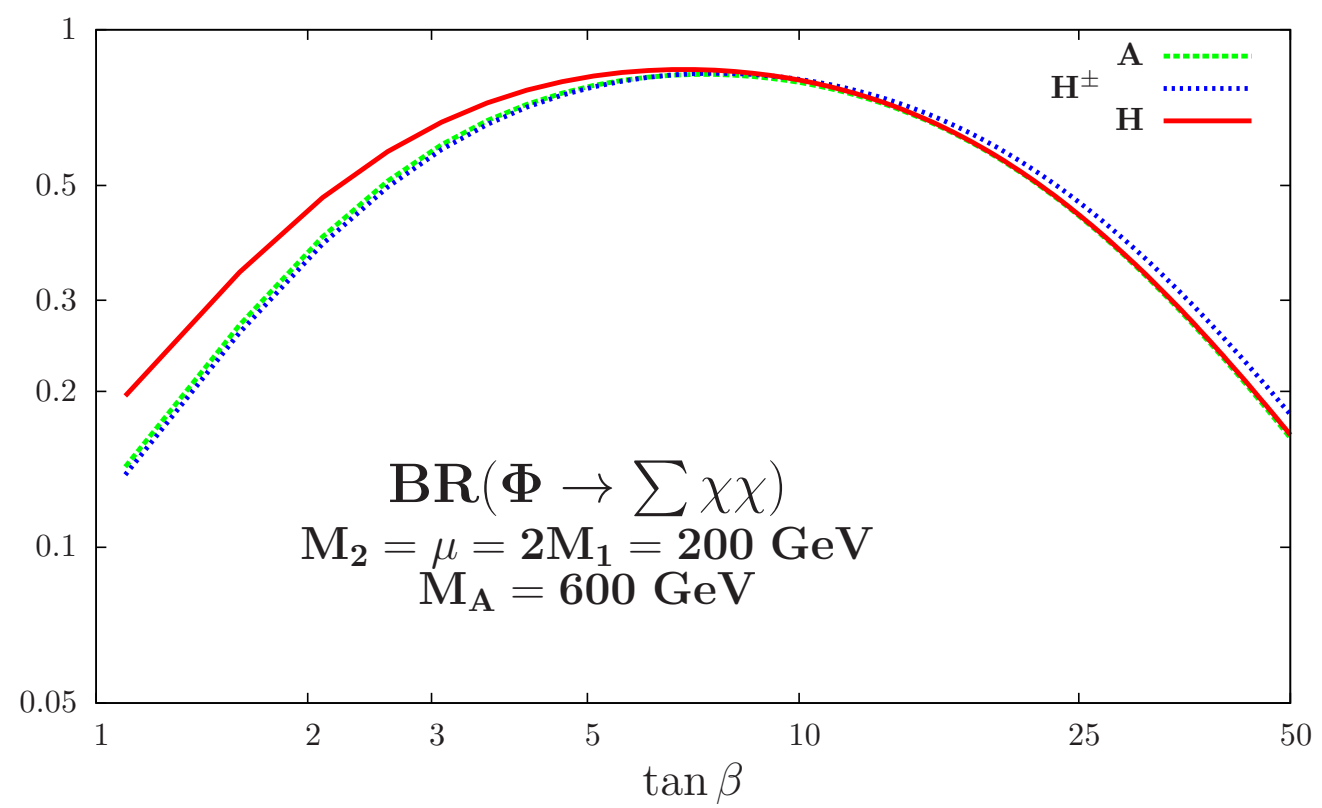

Figure 9. The sum of the branching ratios for the decays of the three heavier MSSM Higgs bosons into charginos and neutralinos as a function of $\tan \beta$ for $M_{A}=600 \mathrm{GeV}$.

the three Higgs particles are indeed similar and that they do not dominate at low nor at high $\tan \beta$. For instance, they are less than $25 \%$ (which is the magnitude of the theoretical uncertainty on the production cross sections) for $\tan \beta \lesssim 2$ and $\tan \beta \gtrsim 30$ as can be seen from the figure. In contrast, the $\chi \chi$ branching rations can be large for intermediate values of $\tan \beta$ when the Higgs couplings to top (bottom) quarks are suppressed (not strongly enhanced) and, for instance, they reach the level of $\approx 70 \%$ at $\tan \beta \approx 5-10$.

Nevertheless, this possibility with large Higgs decay rates into SUSY particles seems unlikely. First, low and comparable values of the wino and higgsino mass parameters $M_{2} \approx \mu \lesssim 300 \mathrm{GeV}$ that would lead to light charginos and neutralinos in the decay products of not too heavy $H / A / H^{ \pm}$bosons, are constrained by the direct searches for these particles at LEP and the LHC [37, 123-125]. In particular, the associated production of the lighter chargino and the next-to-lightest neutralino $q \bar{q} \rightarrow \chi_{1}^{ \pm} \chi_{2}^{0}$ would lead to large cross sections at $\sqrt{s}=8 \mathrm{TeV}$ and the decays $\chi_{1}^{ \pm} \rightarrow W \chi_{1}^{0}$ and $\chi_{2}^{0} \rightarrow Z \chi_{1}^{0}$, with leptonic gauge boson decays, would have significant branching fractions. The search for leptons plus missing energy at the first run of the LHC, in particular the clean trilepton events from the chain $p p \rightarrow \chi_{1}^{ \pm} \chi_{2}^{0} \rightarrow W Z \chi_{1}^{0} \chi_{1}^{0} \rightarrow \ell \ell \ell E_{T}^{\text {mis }}$, imposes severe restrictions on the parameter space. For instance, for $M_{1} \approx 100 \mathrm{GeV}$, the area $\mu \approx M_{2} \lesssim 200 \mathrm{GeV}$ that corresponds to the choice adopted for figure 9 is by now excluded by the LHC data ${ }^{6}$ [125].

\footnotetext{
${ }^{6}$ Note that we have adopted the same choice of gaugino-higgsino parameters as in the benchmark scenario of ref. [40] that has been used to interpret the experimental limits in the $p p \rightarrow \tau \tau$ searches made by the ATLAS and CMS collaborations [35, 36]. Hence, this choice leads to a large branching fraction for Higgs decays into $\chi \chi$ states for $M_{A} \gtrsim 300 \mathrm{GeV}$ and weakens the experimental constraints that can be obtained from $H / A$ searches in the $p p \rightarrow \tau \tau$ process, while it is apparently excluded by direct SUSY searches.
} 
To evade these experimental bounds, one needs either to increase the parameters $\mu_{2} \approx$ $M_{2}$ well above $200 \mathrm{GeV}$ with a consequence that the phase space for the Higgs decays will be limited, or to make that the light $\chi$ states are either pure higgsinos $\left(\mu \ll M_{2}\right)$ or pure gauginos $\left(\mu \gg M_{2}\right)$, which then suppresses the $Z \chi \chi$ couplings e.g. and hence the trilepton signals, leading to Higgs couplings to the kinematically accessible charginos and neutralinos that are too small. Thus, in all these cases, the SUSY decays are suppressed and do not jeopardize the Higgs signals in the standard search channels.

These arguments become stronger in the case where the lightest neutralino $\chi_{1}^{0}$ is forced to be the candidate for the dark matter in the universe, with a cosmological relic density as measured by the WMAP/Planck teams, $0.09 \leq \Omega h^{2} \leq 0.12[126,127]$ with $h$ being the reduced Hubble constant. Traditionally, four regions of the MSSM parameter space have been advocated to fulfill this condition for the LSP neutralino ${ }^{7}$ and we list them below.

i) The so-called "well tempered neutralino" region, with a mixed gaugino-higgsino LSP that allows for significant couplings to gauge and Higgs bosons; this leads to a good LSP annihilation rate into bosonic finals states, $\chi_{1}^{0} \chi_{1}^{0} \rightarrow W W, Z Z, h Z$. For low $M_{2}$ and $\mu$ values, this is the region discussed above that is constrained by the multi-lepton plus missing energy searches.

ii) The bino like neutralino region, $\mu \gg M_{1}$, where the main LSP annihilation channel is into third generation tau leptons, with the exchange of light sleptons in the $t$ channel, $\chi_{1}^{0} \chi_{1}^{0} \rightarrow \tau^{+} \tau^{-}$, and the $\tilde{\tau}$ co-annihilation region in which the lightest $\tilde{\tau}_{1}$ state is almost mass degenerate with the LSP, $m_{\tilde{\tau}_{1}} \approx m_{\chi_{1}^{0}}$, and the correct relic density is provided by the process $\tilde{\tau}_{1} \tilde{\tau}_{1} \rightarrow$ SM particles. If the SUSY scale is high and a kind of universality is assumed for sfermions, the $\tilde{\tau}_{1}$ state will be too heavy and both channels become inoperative.

iii) The regions where the LSP is almost a pure higgsino or gaugino state and hence with small couplings to the $H / A / H^{ \pm}$bosons. The correct cosmological relic density is provided by the co-annihilation of the mass degenerate $\chi_{1}^{ \pm}$and $\chi_{2}^{0}$ states that need to be very heavy (above $1 \mathrm{TeV}$ ) and inaccessible in the decays of $\mathrm{TeV}$ Higgs bosons.

$i v$ ) Finally, there is the Higgs-pole region [128-133] in which an efficient LSP annihilation into SM particles is provided by the exchange of an almost on-shell $A$ boson in the $s$-channel; one thus needs $M_{A} \approx 2 m_{\chi_{1}^{0}}$ (the possibility of $h$-boson exchange $[134,135]$ leading to $m_{\chi_{1}^{0}} \approx 60 \mathrm{GeV}$ is by now unlikely). In the past, the high $\tan \beta$ region was favored and the most discussed annihilation channel was $\chi_{1}^{0} \chi_{1}^{0} \rightarrow A \rightarrow b \bar{b}$. At low $\tan \beta$, a new possibility opens up, the channel $\chi_{1}^{0} \chi_{1}^{0} \rightarrow A \rightarrow t \bar{t}$ which can also lead to the correct relic density.

In figure 10 , we display the areas of the $\left[M_{2}, \mu\right]$ parameter space in which the relic density of the lightest neutralino, calculated using the program micrOMEGAs [136], is as determined by the WMAP/Planck collaborations, i.e. $0.09 \leq \Omega h^{2} \leq 0.12[126,127]$. We

\footnotetext{
${ }^{7}$ For a review see M. Drees and G. Gerbier in ref. [37].
} 

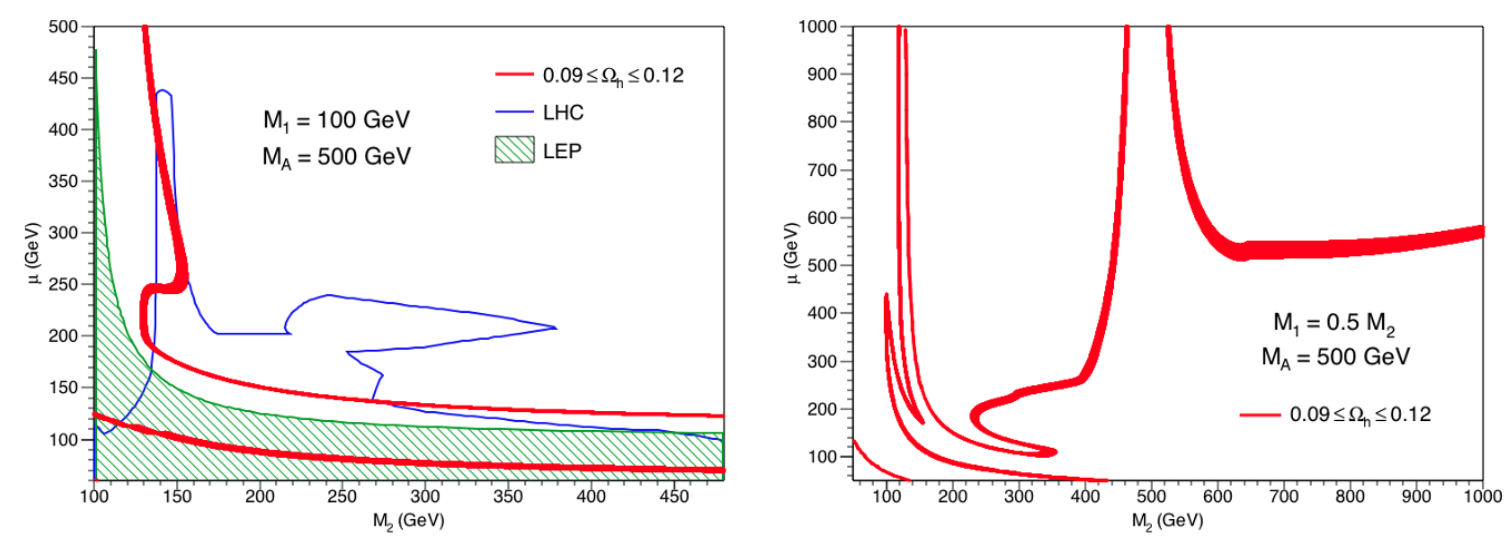

Figure 10. Points in the $\left[M_{2}, \mu\right]$ plane which satisfy the dark matter constraint with $M_{A}=500 \mathrm{GeV}$ and $\tan \beta=2$ for $M_{1}=100 \mathrm{GeV}$ (left) and with the unification condition for the gaugino masses $M_{1}=0.5 M_{2}$ (right). In the left figure, the parameter space excluded by the LEP and LHC direct searches of charginos and neutralinos is indicated.

have chosen $M_{A}=500 \mathrm{GeV}$ and $\tan \beta=2$ for the $h$ MSSM inputs, and assumed very heavy sfermions. In the left plot we have fixed the bino mass to $M_{1}=100 \mathrm{GeV}$, while in the right plot, we used the unification condition $M_{2}=2 M_{1}$. Outside the areas excluded by LEP and LHC ino searches at $M_{1}=100 \mathrm{GeV} \approx m_{\chi_{1}^{0}}$, only two areas lead to a correct relic density: small $M_{2}$ or $\mu$ values $\left(\lesssim 150 \mathrm{GeV}\right.$ ) that allow for $\chi_{1}^{0} \chi_{2}^{0}$ and $\chi_{1}^{0} \chi_{1}^{ \pm}$co-annihilation or a mixed bino-higgsino LSP. Another area opens up if $m_{\chi_{1}^{0}} \approx \frac{1}{2} M_{2} \approx \frac{1}{2} M_{A}$ as can be seen in the right-figure: the $A$ funnel in which the LSP efficiently annihilates through the channel $\chi_{1}^{0} \chi_{1}^{0} \rightarrow A \rightarrow t \overline{\text { }}$. In most of this area, the decay $A \rightarrow \chi_{1}^{0} \chi_{1}^{0}$ is kinematically closed (or phase-space suppressed) and, because $\chi_{1}^{0}$ is the LSP, so are all Higgs decays into superparticles.

Hence, in all cases, the requirement that the lightest neutralino is the dark matter in the universe with the correct relic density makes that the decays of the Higgs bosons into charginos and neutralinos should not occur, or at least should not dominate.

One concludes from all the discussions of this subsection that it is rather unlikely that the SUSY particles make a significant impact in the phenomenology of the MSSM Higgs bosons, either in their virtual contributions to the production and/or decay processes (in particular since the SUSY effects should be larger than the $\approx 25 \%$ theoretical uncertainty that affects the production rates) or in the direct appearance in the decays of the heavier Higgs states. One can thus assume that the superparticles are very heavy and/or too weakly coupled and that they decouple from the MSSM Higgs sector, except of course in the radiative corrections to the CP-even mass matrix eq. (2.1).

This is, in fact, another way of stating the third assumption of the hMSSM discussed in section 2 and one can thus consider this effective approach as a very good benchmark. Ignoring the SUSY effects is a rather reasonable attitude since, besides the tremendous simplifications that the $h \mathrm{MSSM}$ introduces in the description of the Higgs sector, it leads to a straightforward interpretation of the experimental constraints, that do not need to be "de-convoluted" from these complicated effects when they are included. 
Nevertheless, the possibility of light charginos and neutralinos with sizable couplings to the Higgs bosons cannot be totally excluded at the moment. On should therefore continue performing searches for the $H / A / H+\chi \chi$ SUSY decays and other more direct searches for these particles such as the tri-lepton signal events. In the full MSSM with light SUSY particles, all these processes provide complementary information.

\section{The probing of the MSSM parameter space}

\subsection{Interpretation of the fermionic Higgs decay modes in the hMSSM}

As discussed earlier, the most efficient channels that allow to probe the MSSM parameter space at the LHC are the search for charged Higgs bosons coming from top quark decays, the process $t \rightarrow b H^{+}$followed by the decay $H^{+} \rightarrow \tau^{+} \nu$ and its charge conjugate $H^{-}$process, and the search for high mass resonances decaying into tau-lepton pairs, the processes $p p \rightarrow$ $H / A \rightarrow \tau^{+} \tau^{-}$. Both channels have been considered by the ATLAS and CMS collaborations and we briefly summarize below the resulting constraints on the $h$ MSSM.

The CMS $H^{ \pm}$search [34] was performed with the $19.7 \mathrm{fb}^{-1}$ data collected at $\sqrt{s}=8 \mathrm{TeV}$ with the $\tau$-leptons decaying fully hadronically. 95\% CL upper bounds have been set on the product of branching ratios $\mathrm{BR}\left(t \rightarrow b H^{+}\right) \times \mathrm{BR}\left(H^{+} \rightarrow \tau^{+} \nu\right)$ from $1.2 \%$ at $M_{H^{ \pm}}=80 \mathrm{GeV}$ (about the exclusion limit obtained on $M_{H^{ \pm}}$from LEP2 searches [37, 38]) to $0.16 \%$ at $M_{H^{ \pm}}=160 \mathrm{GeV}$ (beyond which phase space effects start to be too penalizing). The search excludes the entire range $M_{H^{ \pm}} \lesssim 140 \mathrm{GeV}$ for all values of $1 \leq \tan \beta \leq 60$. For larger $H^{ \pm}$ masses, the areas where $\tan \beta \approx 8$ at $M_{H^{ \pm}}=140 \mathrm{GeV}$ to $\tan \beta \approx 5-15$ at $M_{H^{ \pm}}=160 \mathrm{GeV}$, in which the $m_{t} / \tan \beta$ component of the $H^{ \pm} t b$ coupling is suppressed while the $m_{b} \tan \beta$ component is not yet enhanced, remain viable at the $95 \% \mathrm{CL}$.

The ATLAS search for $H^{ \pm}$states [33] was also performed with the full $19.5 \mathrm{fb}^{-1}$ data recorded at $\sqrt{s}=8 \mathrm{TeV}$; the same channel as above, i.e. $t \rightarrow b H^{+} \rightarrow b \tau^{+} \nu$ with the taulepton decaying hadronically, has been used. Similar 95\%CL upper limits than CMS have been obtained on the product $\mathrm{BR}\left(t \rightarrow b H^{+}\right) \times \mathrm{BR}\left(H^{+} \rightarrow \tau^{+} \nu\right)$. Compared to the previous limits, a small additional area of the $\left[\tan \beta, M_{H^{ \pm}}\right]$plane, at $\tan \beta \approx 8$ for $M_{H^{ \pm}} \approx 100 \mathrm{GeV}$ and to $\tan \beta \approx=6-10$ at $M_{H^{ \pm}} \approx 90 \mathrm{GeV}$, remains unexcluded by the ATLAS analysis, as a result of the presence of a large $t \bar{t}, W / Z+$ jet backgrounds in this mass bin.

These limits can be turned into bounds in the $\left[M_{A}, \tan \beta\right]$ parameter space assuming the usual relation $M_{H^{ \pm}}^{2}=M_{A}^{2}+M_{W}^{2}$. This is what is illustrated by the dark blue area of figure 11 in which the constraints on the $\left[M_{A}, \tan \beta\right]$ plan are shown: we take the limits on $\operatorname{BR}\left(t \rightarrow b H^{+}\right) \times \mathrm{BR}\left(H^{+} \rightarrow \tau^{+} \nu\right)$, that we calculate using the program SDECAY [137], and interpret them in the $h \mathrm{MSSM}$. We see that in our case, the entire area $M_{A} \lesssim 140 \mathrm{GeV}$ is excluded at low $\tan \beta$ values, reducing to $M_{A} \lesssim 130 \mathrm{GeV}$ at high $\tan \beta$. In contrast to ATLAS and CMS, which use different means to calculate the product of branching ratios, we do not have the holes at the extreme values of $M_{H^{ \pm}}$.

In the previous references, both experiments performed also searches for heavier $H^{ \pm}$ states with $M_{H^{ \pm}} \gtrsim m_{t}+m_{b} \approx 180 \mathrm{GeV}$, by considering the process $p p \rightarrow H^{ \pm} t(b)$ with again $H^{+} \rightarrow \tau^{+} \nu$. The areas $\tan \beta \gtrsim 45(60)$ at $M_{H^{ \pm}} \approx 200(250) \mathrm{GeV}$ are excluded by the CMS collaboration (the ATLAS group assumed $\operatorname{BR}\left(H^{+} \rightarrow \tau^{+} \nu\right)=100 \%$ in this area while it 


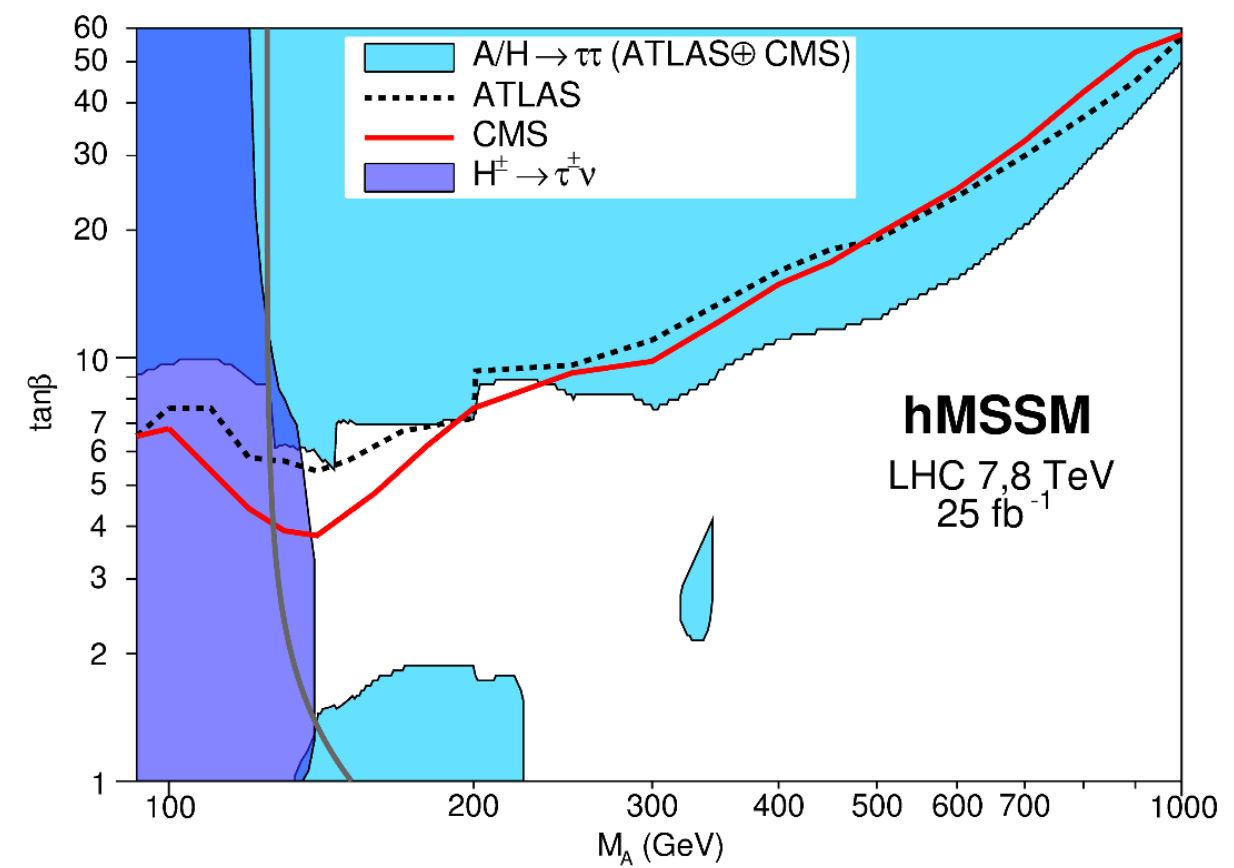

Figure 11. The $\left[\tan \beta, M_{A}\right]$ plane in which the $p p \rightarrow H / A \rightarrow \tau^{+} \tau^{-}$(light blue) and $t \rightarrow b H^{+} \rightarrow b r \nu$ (dark blue) search limits of ATLAS and CMS are combined and interpreted within the $h$ MSSM. The red-solid (blue-dotted) line is for the ATLAS (CMS) observed limit only in the $M_{h}^{\max }$ scenario and the region at the left of the black-solid line is the one where the $h$ MSSM is ill-defined.

should be only 10\%). These limits are much less powerful than those obtained from the $\tau \tau$ search as will be seen shortly. However, there was in interesting CMS search in the channel $H^{+} \rightarrow t b$ performed with $19.7 \mathrm{fb}^{-1}$ data at $\sqrt{s}=8 \mathrm{TeV}[110]$. Limits on $\sigma\left(p p \rightarrow t b H^{ \pm}\right)$ assuming $\mathrm{BR}\left(H^{+} \rightarrow t b\right)=1$ have been set and surprisingly, one is not far from being sensitive to the very low $\tan \beta$ area and with about a factor of two more data, one would have probed $\tan \beta \approx 1$ for $M_{H^{ \pm}} \approx 200 \mathrm{GeV}$.

We note that these limits from $H^{ \pm}$searches exclude a substantial part of the low $\left[\tan \beta, M_{A}\right]$ area in which the $h$ MSSM is ill defined, i.e. the region at the left of the thick black solid line in figure 11. The exclusion is valid in this area as all the ingredients used to obtain the limits depend only on $M_{H^{ \pm}}$and $\tan \beta$ and do not involve the CP-even Higgs parameters $M_{H}$ or $\alpha$ that were undefined. The only assumption is that the relation $M_{H^{ \pm}}^{2}=M_{A}^{2}+M_{W}^{2}$ remains valid. Hence, one problematic issue within the $h$ MSSM is in a sense partly solved by these "model-independent" exclusion limits (we do not address here the theoretical issue of the validity of the entire model at $\tan \beta$ values close to unity).

The most important search mode in the MSSM is certainly the $p p \rightarrow H / A \rightarrow \tau \tau$ channel. The ATLAS collaboration has searched for this signal using the $19.5-20.3 \mathrm{fb}^{-1}$ data collected at $8 \mathrm{TeV}$ [35] while CMS has used the full $24.6 \mathrm{fb}^{-1}$ data collected at $7+8 \mathrm{TeV}[36]$. Both collaborations consider the leptonic $\left(\tau_{e} \tau_{\mu}\right)$, semi-leptonic $\left(\tau_{\ell} \tau_{\text {had }}\right)$ and hadronic $\left(\tau_{\text {had }} \tau_{\text {had }}\right) \tau$ decays and CMS also considers the case where an additional $b$-quark is present in the final state. Limits at the 95\% CL on $\sigma\left(p p \rightarrow \tau^{+} \tau^{-}\right)$as a function of the invariant mass $M_{\tau \tau}$ of the tau-lepton pair have been given by the two experiments. 
Our procedure to interpret these limits in the $h \mathrm{MSSM}$ is as follows. First, we combine the ATLAS and CMS 95\% CL exclusion limits on $\sigma\left(p p \rightarrow \tau^{+} \tau^{-}\right)$. We then compare them with the numbers that we obtain for the rates, namely the $H / A$ production cross sections calculated with the programs HIGLU $[103,104]$ and SusHi [102] and the branching ratios $\operatorname{BR}(H / A \rightarrow \tau \tau)$ calculated using HDECAY $[82,83]$. These are derived assuming that the parameters $M_{H}$ and $\alpha$ given by eqs. (2.6) and ignoring all possible SUSY effects. For a given $\left[\tan \beta, M_{A}\right.$ ] point, if in a window of $\Delta M_{\tau \tau}=40 \mathrm{GeV}$ centered on $M_{A}$ we have either the observed $h$ boson or the heavier $H$ state, we include their contribution to the signal.

The result is shown figure 11 by the light blue areas of the $\left[M_{A}, \tan \beta\right]$ plane and is truly impressive. The largest area is the one that excludes all values above $\tan \beta \approx 8$ for $M_{A} \lesssim 300 \mathrm{GeV}$, extending to $\tan \beta \approx 20$ (40) for $M_{A} \lesssim 700$ (900) GeV.

The ATLAS and CMS observed limits, when interpreted in the $M_{h}^{\max }$ benchmark scenario, are also displayed in figure 11 . We observe that below $M_{A} \approx 200 \mathrm{GeV}$, our limit is less restrictive, the reason being that we do not make use of any refinements in order to treat the regions in which the three Higgs bosons have a comparable mass and, also, to deal with the observed signal of the lighter $h$ boson. In contrast, our limit is stronger at masses above $M_{A} \approx 300 \mathrm{GeV}$, the reason being that while in our case there is no SUSY decays of the Higgs bosons, the $M_{h}^{\max }$ scenario leads to $H / A$ decays into charginos and neutralinos that are significant. Indeed, for the gaugino and higgsino mass parameters of the $M_{h}^{\max }$ benchmark (and the slightly modified ones), $\mu=M_{2}=2 M_{1}=200 \mathrm{GeV}$, the LSP has a mass of $m_{\chi_{1}^{0}} \approx 100 \mathrm{GeV}$ while the heavier neutralinos and the charginos have masses of the order of $\approx 200 \mathrm{GeV}$, so that many SUSY decays occur starting from $M_{A} \approx 300 \mathrm{GeV}$ and all of them will be present for $M_{A} \approx 500 \mathrm{GeV}$ and above. These decays will have substantial branching fractions, in particular in the intermediate $\tan \beta \approx 10$ range where they become dominant, as can be seen from figure 9 . Hence, the $H / A \rightarrow \tau \tau$ branching ratios are suppressed in this this case, resulting in a weaker exclusion limit (which is unfortunate since this parameter configuration is almost certainly excluded by the direct searches [125]).

A second step is to extrapolate the ATLAS and CMS limits to the low $\tan \beta$ region, which as discussed earlier, can be described within the $h \mathrm{MSSM}$ approach in contrast to the benchmark scenarios used by the collaborations. Two islands were discovered during the exploration. A first and substantial area is at very low $\tan \beta$ and $M_{A}, \tan \beta \lesssim 2$ and $M_{A} \lesssim 230 \mathrm{GeV}$. Here, because part of the area is ill defined, we consider only the production and the decay of the $A$ state that depend only on $\tan \beta$ and $M_{A}$ as the decay channel $A \rightarrow h Z$, which introduces a dependence on the angle $\alpha$ through the $A h Z$ coupling, is not yet kinematically open. In this domain, as discussed earlier, both the $g g \rightarrow A$ cross section (dominated by the top-quark loop) and the branching ratio for the decay $A \rightarrow \tau \tau$ (which, together with the one for $b \bar{b}$ and $c \bar{c}$, is the only significant one to occur) is substantial. Hence, despite of the fact that we have only one resonance, the cross section times branching fraction is large enough to generate an observable signal. The excluded area from this search removes the small residual part of the ill-defined $h$ MSSM region, that was left after imposing the exclusion limit from the $H^{ \pm}$searches discussed above.

More surprising at first sight, we discovered another smaller island at $M_{A} \approx 350 \mathrm{GeV}$ and $\tan \beta \approx 2-4$. It turns out that, around the $t \bar{t}$ threshold, there is a very strong increase 
of the $g g \rightarrow A$ amplitude as the form-factor $\operatorname{Re}\left(A_{1 / 2}^{A}\right) \approx 5$ is maximal at the $2 m_{t}$ threshold, ${ }^{8}$ $\tau=M_{A}^{2} / 4 m_{t}^{2} \approx 1$. At the same time, $\operatorname{BR}(A \rightarrow \tau \tau) \approx$ is substantial being a few percent, as the other decays except for $b \bar{b}$ are slightly suppressed, $A \rightarrow h Z$ by the coupling $\approx \cos (\beta-\alpha)$ and $A \rightarrow t \bar{t}$ by phase space effects (only the three-body decay channel is kinematically open and it is suppressed). Hence, there would have been a substantial surplus of events from the $g g \rightarrow A \rightarrow \tau \tau$ process in this limited area that is excluded by the search.

\subsection{Interpretation of the bosonic Higgs decay modes in the hMSSM}

We now turn to the constraints that can be imposed on the $\left[\tan \beta, M_{A}\right]$ plane by considering the bosonic decay channels of the heavier $H$ and $A$ states. In contrast to the $H / A \rightarrow \tau \tau$ and $H^{ \pm} \rightarrow \tau \nu$ searches, no interpretation of these modes has been done in the context of the MSSM by the ATLAS and CMS collaborations. In the following, we will therefore adapt the constraints that have been obtained either in the context of the SM but with a heavier Higgs state than the observed one, or in extensions of the SM other than the MSSM. We will focus on the experimental analyses that provide the most stringent constraints.

The massive gauge boson channels $H \rightarrow W W, Z Z$ have not been discussed in the context of the MSSM but important information can be borrowed from those performed for a heavy SM Higgs boson. For instance, a search of the $H \rightarrow W W \rightarrow \ell \ell \nu \nu$ and $H \rightarrow Z Z \rightarrow 4 \ell$ channels have been conducted in the SM with the full event sample recorded at the LHC first phase, i.e. $4.9 \mathrm{fb}^{-1}$ at $\sqrt{s}=7 \mathrm{TeV}$ and $19.4 \mathrm{fb}^{-1}$ at $\sqrt{s}=8 \mathrm{TeV}$ for $H \rightarrow W W^{*}$ [45] and $5.1 \mathrm{fb}^{-1}$ at $\sqrt{s}=7 \mathrm{TeV}$ and $19.7 \mathrm{fb}^{-1}$ at $\sqrt{s}=8 \mathrm{TeV}$ for $H \rightarrow Z Z^{*}$ [46]. The high mass range was analyzed and the events corresponding to the observed state with a mass of $125 \mathrm{GeV}$ were considered as a background. In the $Z Z$ channel, an additional CMS analysis in the $H \rightarrow Z Z^{*} \rightarrow 2 \ell 2 q$ channel has been made with the $19.6 \mathrm{fb}^{-1}$ data collected at $8 \mathrm{TeV}$ [138]. All these analyses exclude a significant area in the $h$ MSSM parameter space at low and moderate $\tan \beta$.

In the case of the $H \rightarrow W W \rightarrow \ell \ell \nu \nu$ CMS search, when all production channels are included (there is a dominance of the $g g \rightarrow H$ mode of course) and the various final state topologies are summed up, a Higgs particle with a SM-like coupling to gauge bosons is excluded from $M_{H} \approx 200 \mathrm{GeV}$ to $\approx 600 \mathrm{GeV}$. The $95 \% \mathrm{CL}$ upper limit as a function of $M_{H}$ and relative to the SM expectation can be easily turned into an exclusion area in the $\left[M_{A}, \tan \beta\right]$ plane by considering the production and decay rates of the MSSM $H$ state discussed in section 3. The result is shown in figure 12 where the area excluded by this search, interpreted in the context of the $h \mathrm{MSSM}$, is depicted in dark green.

The exclusion area starts at relatively high $\tan \beta$ values, $\tan \beta \gtrsim 10$, and light $A$, $M_{A} \approx 140 \mathrm{GeV}$ (below this limit, we enter the domain in which the model is ill defined, a domain that extends to $M_{A} \approx 160 \mathrm{GeV}$ and $\tan \beta \approx 1$ ) where one has an $H$ state with a mass $M_{H} \gtrsim 160 \mathrm{GeV}$ and a coupling $g_{H V V}=\cos (\beta-\alpha)$ that is not very small as shown in the right-hand side of figure 3 , allowing for substantial $H$ production times decay rates. For

\footnotetext{
${ }^{8}$ In fact the NLO QCD corrections to $\sigma(g g \rightarrow A)$ introduce a Coulomb singularity exactly at threshold [92]. However, we have checked that the rate increase in this new area is not due to this unphysical feature.
} 
$\tan \beta \approx 1$, the excluded region extends to $M_{A} \approx 250 \mathrm{GeV}$, when other decay channels such as $H \rightarrow h h$ and even $H \rightarrow t \bar{t}$ open up and suppress the massive gauge boson decay modes.

As a result of its clean final state and despite of the low statistics, the $H \rightarrow Z Z \rightarrow 4 \ell$ search turns out to be more constraining at high mass and excludes a SM-like Higgs boson up to $M_{H} \approx 800 \mathrm{GeV}$ (with a search domain extending to $1 \mathrm{TeV}$ ). While for low $M_{H}$ values, $H \rightarrow Z Z$ is less powerful than the companion $H \rightarrow W W$ mode as a consequence of the reduced phase space, it clearly becomes the leading channel for $M_{H} \gtrsim 250 \mathrm{GeV}$. In fact, because of the higher statistics, the most severe constraint is obtained in the combination of the $H \rightarrow Z Z \rightarrow 4 \ell, 2 \ell 2 \nu, 2 \ell 2 q$ topologies that was performed in ref. [138]. Here, the 95\% CL exclusion of a Higgs state with SM-like couplings extends to a mass close to $1 \mathrm{TeV}$.

The area excluded at $95 \%$ CL by the non observation of these $Z Z$ final states at the LHC outside the $M_{h} \approx 125 \mathrm{GeV}$ mass window is given by the light green area of figure 12 . It extends from $M_{A} \approx 160 \mathrm{GeV}$ to $M_{H} \approx 280 \mathrm{GeV}$ and concerns all values $\tan \beta \lesssim 5$. An additional small area around $M_{A} \approx 300 \mathrm{GeV}$ and $\tan \beta \lesssim 2$, in which $M_{H}$ is close to the $2 m_{t}$ threshold and the gluon-fusion amplitude $A_{1 / 2}^{H}$ is maximal thus enhancing the $g g \rightarrow H$ cross section, is also excluded.

One should note that in a dedicated MSSM search, not only this $H \rightarrow Z Z$ channel but also the $H \rightarrow W W$ mode will lead to more effective constraints as the SM and MSSM Higgs particles have total decay widths that are completely different at high masses [10]. Indeed, while the SM state would have been a very wide resonance, the MSSM $H$ boson is a relatively narrow resonance as shown in figure 5, allowing to select smaller bins for the $V V$ invariant masses that lead to a more effective suppression of the various backgrounds.

The resonant $H \rightarrow h h$ channel, which is important in the mass range between 250 and slightly above $350 \mathrm{GeV}$ has been considered by both the ATLAS and CMS collaborations with the $\approx 20 \mathrm{fb}^{-1}$ of data collected at $\sqrt{s}=8 \mathrm{TeV}$. The main focus was on the $\gamma \gamma b \bar{b}$ signature $[47,48]$ but additional searches in the $4 b$-quark final state have been recently reported $[139,140]$. However, neither collaborations has interpreted the 95\%CL exclusion limits in these channels in the context of the MSSM, the main reason being again that the low $\tan \beta$ area in which these signals occur is not theoretically accessible in the usual benchmark scenarios used for the MSSM Higgs sector.

The interpretation is however straightforward in the $h \mathrm{MSSM}$ as the trilinear selfcoupling $\lambda_{H h h}$ that controls the $H \rightarrow h h$ decay rate is simply given, as shown in eq. (2.10), in terms of the angles $\alpha, \beta$ and the radiative correction matrix element $\Delta M_{22}^{2}$ that is fixed in terms of $\tan \beta$ and $M_{A}$ if the constraint $M_{h}=125 \mathrm{GeV}$ is used. We have adapted the constraints from these analyses to the $h \mathrm{MSSM}$ case and the resulting excluded domain in the $\left[\tan \beta, M_{A}\right]$ plane is shown in purple in figure 12 . It covers the very low $\tan \beta$ region, $\tan \beta \lesssim 2$, for the mass range between $M_{A} \approx 270 \mathrm{GeV}$ (which implies $M_{H} \gtrsim 250 \mathrm{GeV}$ for these low $\tan \beta$ values) and $M_{A} \approx 330 \mathrm{GeV}$, i.e. slightly before the $2 m_{t}$ threshold.

Similarly to the previous channel, the $A \rightarrow h Z$ mode has only been considered in the context of two Higgs doublet models [69] and not in the MSSM. A CMS analysis considered the final state $b \bar{b} \ell^{+} \ell^{-}$with the $\approx 20 \mathrm{fb}^{-1}$ collected in 2012 at $8 \mathrm{TeV}$ [49]. A search of both the $A \rightarrow h Z$ and $H \rightarrow h h$ channels has been performed by CMS again in the multi-lepton and eventually photon finale states [141]. The impact of the 95\%CL exclusion limits of 


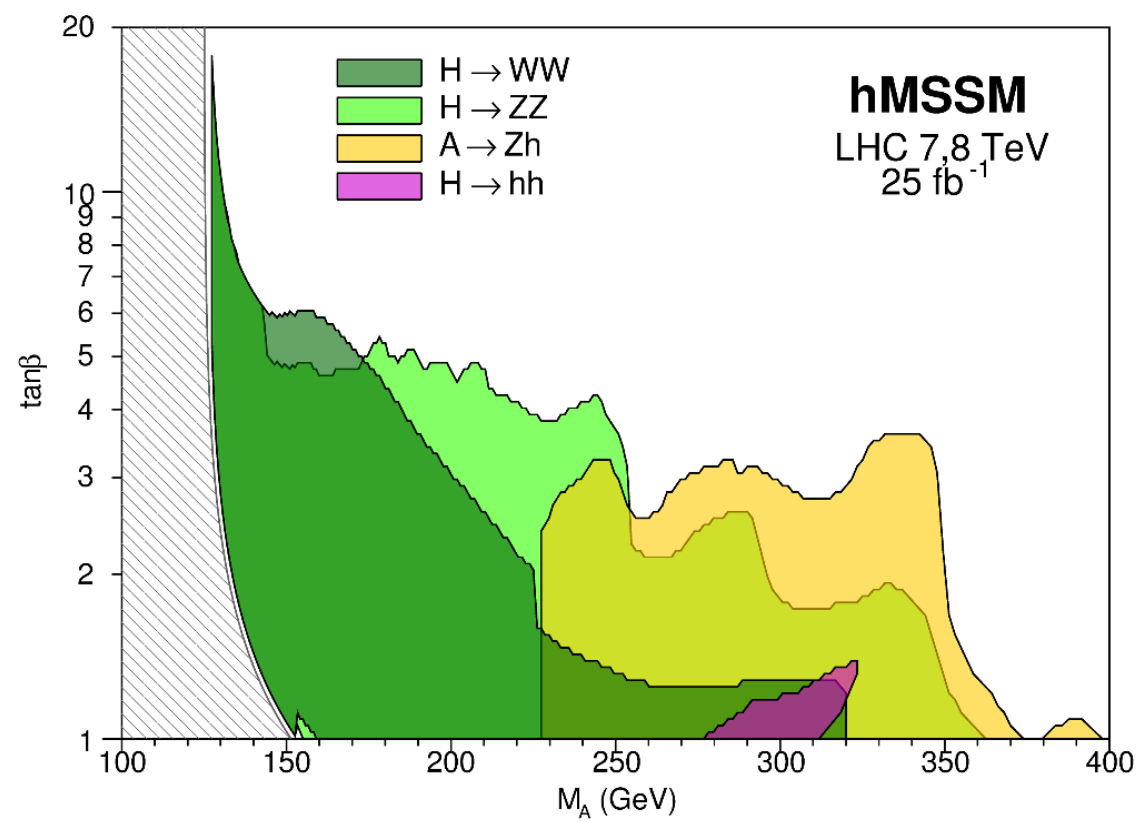

Figure 12. Constraints in the $\left[\tan \beta, M_{A}\right]$ plane of the $h$ MSSM from search at the LHC for a heavy CP-even boson decaying into $W W, Z Z, h h$ and a heavy CP-odd boson decaying into $h Z$ final states. The searches are for $\sqrt{s}=7+8 \mathrm{TeV}$ c.m. energy and $25 \mathrm{fb}^{-1}$ of accumulated data. The dashed area is the one that is ill-defined in the $h \mathrm{MSSM}$.

these studies, when interpreted in the context of the $h \mathrm{MSSM}$, is illustrated by the yellow area of Figure 12 . The ranges $\tan \beta \lesssim 3$ and $M_{A} \approx 230-350 \mathrm{GeV}$ should be in principle excluded with the present data.

\subsection{Summary of the constraints at $8 \mathrm{TeV}$ and projections for $14 \mathrm{TeV}$}

Wrapping up the discussion up to this point, the impact on the $\left[M_{A}, \tan \beta\right]$ plane of the searches in the fermionic Higgs decays $H / A \rightarrow \tau \tau$ and $H^{ \pm} \rightarrow \tau \nu$ and in the bosonic ones $H \rightarrow W W, Z Z, h h$ and $A \rightarrow Z h$ performed by the ATLAS and CMS collaborations at $\sqrt{s}=7+8 \mathrm{TeV}$ with up to $\approx 25 \mathrm{fb}^{-1}$ data are combined in figure 13 . The outcome is very impressive. The high $\tan \beta \gtrsim 10$ is entirely excluded for $M_{A} \lesssim 500 \mathrm{GeV}$ by the $\tau \tau$ searches. The range $\tan \beta \lesssim 4$ is excluded for $M_{A} \lesssim 250 \mathrm{GeV}$ by the $H \rightarrow W W, Z Z$ channels. For $\tan \beta \lesssim 2$, the excluded domain extends to $M_{A} \lesssim 350 \mathrm{GeV}$ when the channels $H \rightarrow h h$ and $A \rightarrow h Z$ are considered. The entire low $M_{A}$ region, $M_{A} \lesssim 140 \mathrm{GeV}$, is excluded by the $H^{+} \rightarrow \tau \nu$ search for any value of $\tan \beta$. An additional portion of this low $M_{A}$ area is excluded by the $A \rightarrow \tau \tau$ search for low $\tan \beta$ values.

In fact, the entire area in which the $h \mathrm{MSSM}$ is not mathematically defined, and which is delineated by the solid line in the figure, is excluded by these $H^{ \pm}$and $A$ searches that do not involve the undefined CP-even $H$ boson mass $M_{H}$ and the mixing angle $\alpha$.

These constraints, if no new signal is observed, can be vastly improved at the next phase of the LHC with a center of mass energy up to $\sqrt{s}=14 \mathrm{TeV}$ and with one or two orders of magnitude accumulated data. More optimistically, this implies that the $2 \sigma$ sensitivity for a heavier MSSM Higgs boson will be drastically enhanced at the next LHC phase. Starting 


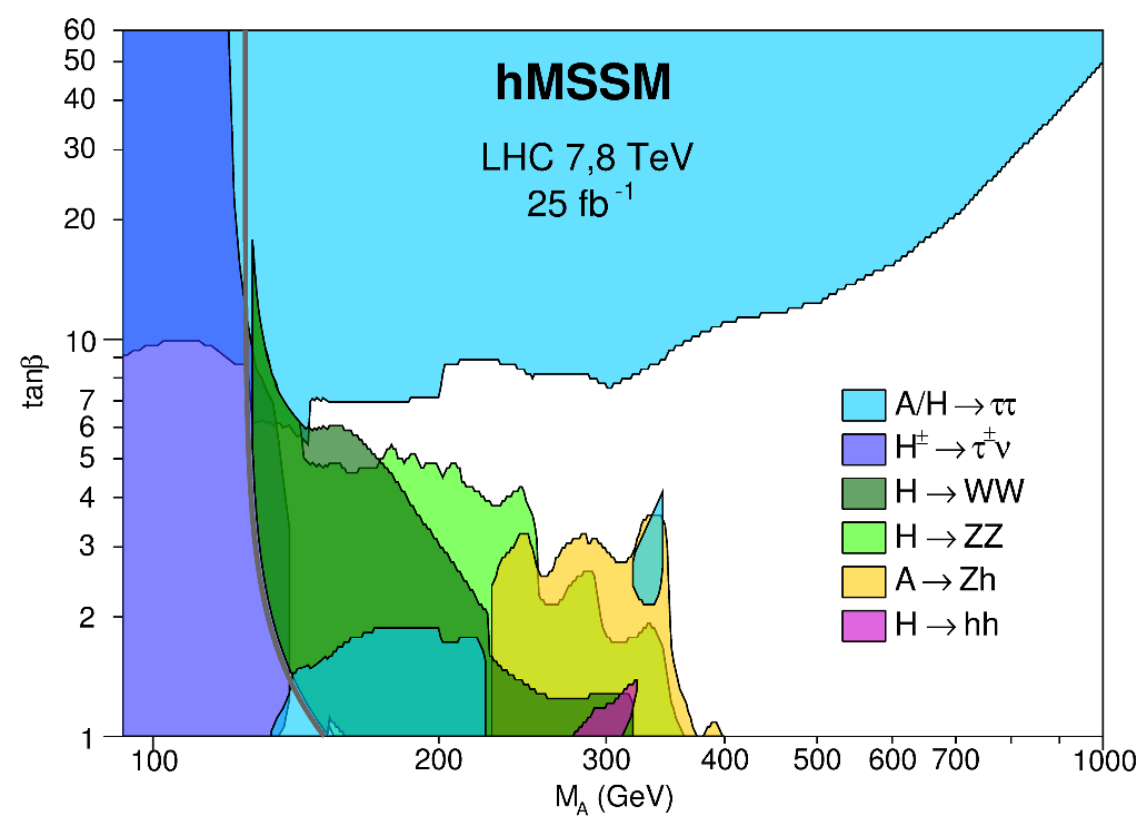

Figure 13. The combined constraints in the $\left[\tan \beta, M_{A}\right]$ plane of the $h$ MSSM from searches at the LHC for the heavier $H, A$ and $H^{ \pm}$bosons decaying into either fermionic or bosonic final states. All the searches performed at c.m. energies up to $\sqrt{s}=8 \mathrm{TeV}$ and $25 \mathrm{fb}^{-1}$ data are included.

from the expected median 95\%CL exclusion limits that have been given by the ATLAS and CMS collaborations in the various searches performed at $8 \mathrm{TeV}$ with $\approx 20 \mathrm{fb}^{-1}$, we have made an extrapolation to this next LHC phase with $\sqrt{s}=14 \mathrm{TeV}$ and $300 \mathrm{fb}^{-1}$ data. We have naively assumed that the sensitivity will simply scale with the square root of the number of expected events and did not include any additional systematical effect. This comes from the observation that the results of the experimental analyses are limits on the signal cross section at a given c.m. energy for a given resonance mass bin, $R_{\sqrt{s}}^{S}\left(M_{A}\right)$, for a channel that is subject to a given background rate $R_{\sqrt{s}}^{B}\left(M_{A}\right)$ at this mass bin, when the integrated luminosity is fixed at a value $\mathcal{L}_{\sqrt{s}}$. Knowing the sensitivity limit $R_{8}^{S}\left(M_{A}\right)$ at $\sqrt{s}=8 \mathrm{TeV}$, one derives the associated limit at $\sqrt{s}=14 \mathrm{TeV}$ using

$$
R_{14}^{S}\left(M_{A}\right)=\sqrt{\mathcal{L}_{8} / \mathcal{L}_{14}} \times \sqrt{R_{14}^{B}\left(M_{A}\right) / R_{8}^{B}\left(M_{A}\right)} \times R_{8}^{S}\left(M_{A}\right) .
$$

Having the knowledge of only the signal cross sections $\sigma_{\sqrt{s}}^{S}\left(M_{A}\right)$ for the various points and not the corresponding background rates, we assume that the latter simply and very naively scale like the signal cross sections. This is the case of some channels of interest, such as $g g \rightarrow H / A \rightarrow t \bar{t}$ whose main background is $g g \rightarrow t \bar{t}$ and as both are $g g$ initiated processes, they roughly scale with the $g g$ luminosity at higher energies. However, for many other channels such as $H \rightarrow W W, Z Z$ or $A / H \rightarrow \tau \tau$, the irreducible background is mostly due to $q \bar{q}$ annihilation which increases more slowly with energy than the initiated $g g$ signal processes. This makes our approach rather conservative.

With this assumption, one obtains for the sensitivity at $\sqrt{s}=14 \mathrm{TeV}, R_{14}^{S}\left(M_{A}\right)$, needed to set the exclusion limit, that we turn into a 95\%CL sensitivity, for a given $M_{A}$

$$
R_{14}^{S}\left(M_{A}\right) \approx \sqrt{\mathcal{L}_{8} / \mathcal{L}_{14}} \times \sqrt{\sigma_{14}^{S}\left(M_{A}\right) / \sigma_{8}^{S}\left(M_{A}\right)} \times R_{8}^{S}\left(M_{A}\right) .
$$



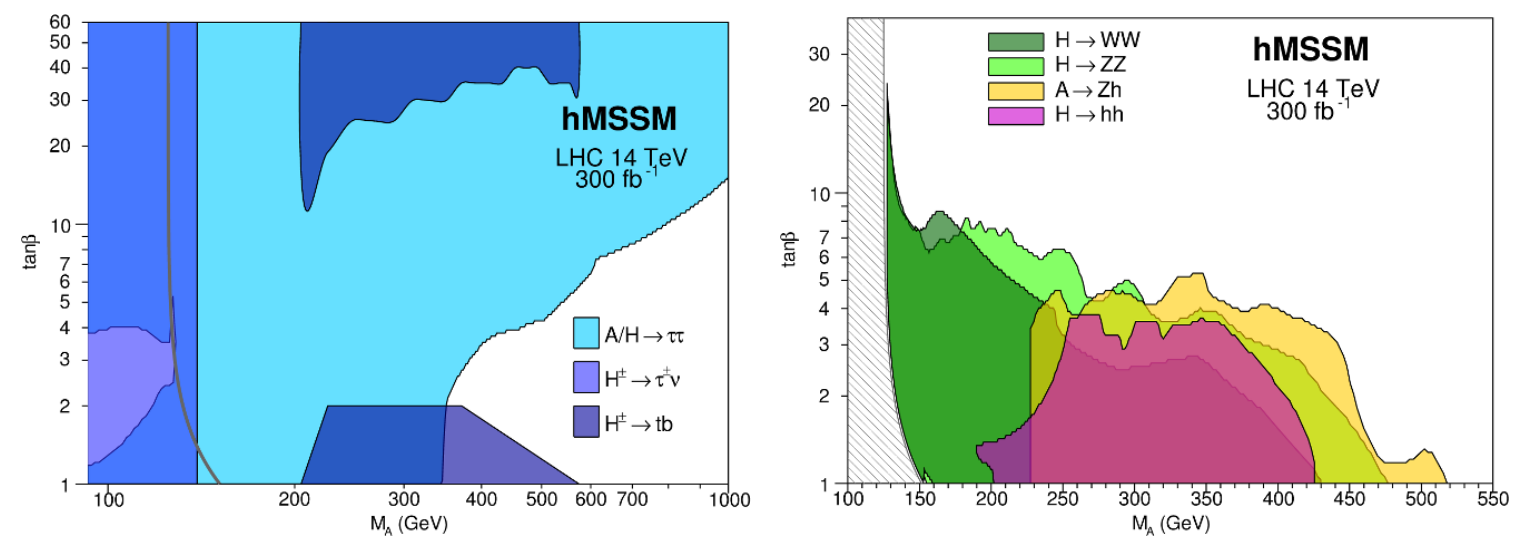

Figure 14. Projections for the LHC with $\sqrt{s}=14 \mathrm{TeV}$ and $300 \mathrm{fb}^{-1}$ data for the $2 \sigma$ sensitivity in the $h \mathrm{MSSM}\left[\tan \beta, M_{A}\right]$ plane from the search for $A / H^{ \pm}$states in their fermionic decays (left) and $A / H$ states in their bosonic decays (right). The same color code as at $\sqrt{s}=8 \mathrm{TeV}$ has been used and, for the fermionic channels, we add a constraint from the $H^{+} \rightarrow t b$ mode depicted in dark blue.

The output of this procedure is presented in the $\left[\tan \beta, M_{A}\right] h \mathrm{MSSM}$ plane in figures 14 for the fermionic (left) and bosonic (right) Higgs search channels. In the former case, we have included in addition the channel $p p \rightarrow t b H^{+} \rightarrow t b t b$ which now shows some sensitivity a low $\tan \beta$ and not too high $M_{H^{ \pm}}$values. The combined expected $95 \% \mathrm{CL}$ sensitivities are shown in figure 15 and, as can be seen, a vast improvement of the current sensitivity to the MSSM parameter space is foreseen in all channels. This is particularly the case of the $A / H \rightarrow \tau \tau$ channels which alone, closes the entire region below $M_{A} \lesssim 350 \mathrm{GeV}$ for any $\tan \beta$ value, while the $H \rightarrow W W, Z Z$ modes which show sensitivity up to $M_{A} \approx 600 \mathrm{GeV}$ at very low $\tan \beta$. In the Higgs mass range in which they are relevant, i.e. below the $t \bar{t}$ threshold, the channels $H \rightarrow h h$ and $A \rightarrow h Z$ start to probe rather high $\tan \beta$ values, $\tan \beta \approx 4$ and $\tan \beta \approx 6$, respectively.

Nevertheless, there will remain an area of the $h$ MSSM parameter space, at $\tan \beta \lesssim 4$ and masses above $M_{A} \approx 400 \mathrm{GeV}$ to name it, which will not be accessible by the channels that have been considered so far in the search of the heavier $H / A$ and $H^{ \pm}$states. To probe this area, the high luminosity option of the LHC with $\mathcal{L}=3 \mathrm{ab}^{-1}$ data or a higher energy $p p$ collider, such as the presently discussed Fcc-pp at $\sqrt{s} \approx 100 \mathrm{TeV}$ will be necessary. However, as it was discussed in many instances in this paper, this virgin area is the ideal territory to perform searches in the $g g \rightarrow H / A \rightarrow t \bar{t}$ channel to which we turn our attention now.

\subsection{Including the $p p \rightarrow H / A \rightarrow t \bar{t}$ channel}

As it was discussed at length in the previous section, for low $\tan \beta$ and high $M_{A}$ values, the decay modes $H / A \rightarrow t \bar{t}$ of the heavier MSSM scalar and pseudoscalar Higgs states will largely become the dominant ones while the $g g \rightarrow H / A$ cross sections are still substantial thanks to the large Higgs coupling to the top quarks that mediate the production process. Hence, the search for resonances decaying into $t \bar{t}$ final states will be mandatory in order 


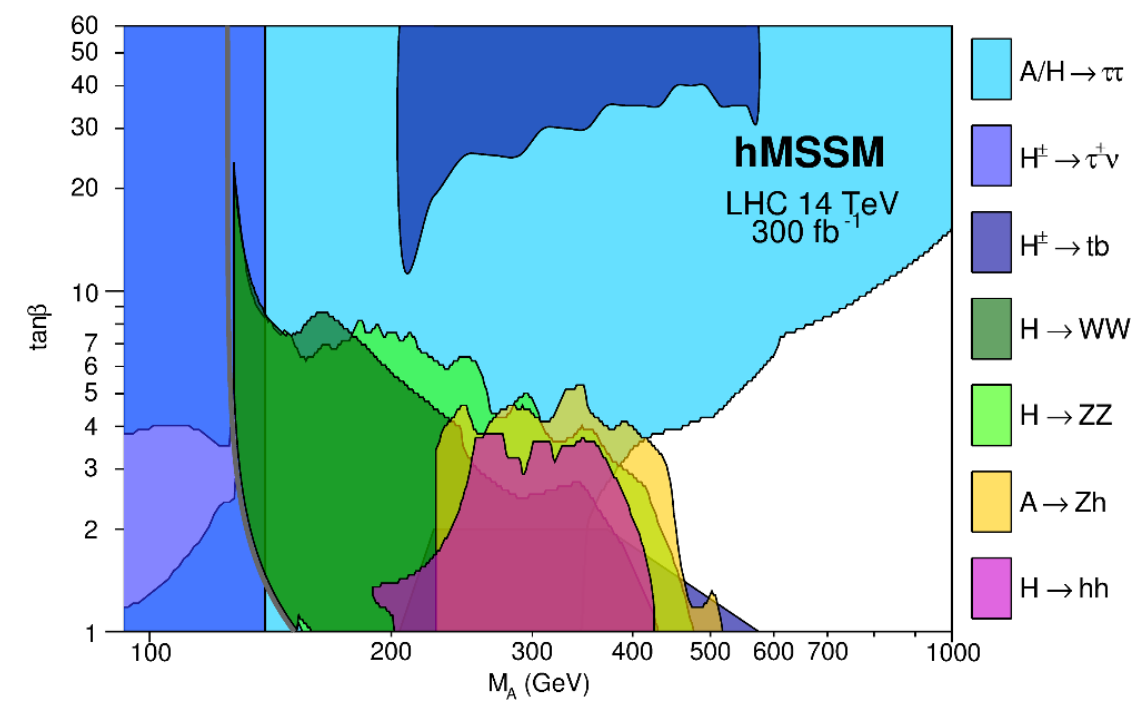

Figure 15. Projections for the LHC with $\sqrt{s}=14 \mathrm{TeV}$ and $300 \mathrm{fb}^{-1}$ data for the $2 \sigma$ sensitivity in the $h$ MSSM $\left[\tan \beta, M_{A}\right.$ ] plane when the searches for the $A / H / H^{ \pm}$states in their fermionic and bosonic decays are combined.

to probe these areas of the $\left[M_{A}, \tan \beta\right]$ parameter space at the LHC. However, a peak in the invariant mass distribution of the $t \bar{t}$ system, that one generally expects in the narrowwidth approximation, is not the only signature of a Higgs resonance in this case. Indeed, the $g g \rightarrow H / A$ signal will interfere with the QCD $t \bar{t}$ background which, at LHC energies, is mainly generated by the gluon-fusion channel, $g g \rightarrow t \bar{t}$. The signal-background interference will depend on the CP nature of the $\Phi=H / A$ boson and on its mass and total decay width; it can be either constructive or destructive, leading to a rather complex signature with a peak-dip structure of the $t \bar{t}$ invariant mass distribution.

These aspects are known since already some time and have especially been discussed in the context of a heavy SM Higgs state [142-144] and, hence, for the CP-even Higgs case. The slightly more involved MSSM situation, as there are one CP-even and one CP-odd resonances that are close in mass, has been addressed only in a very few places; see for instance refs. [145-149]. Dedicated analyses have been performed at the parton-level only and do not make use of recent developments like boosted heavy quark techniques [150] that could allow to enhance the observability of the Higgs signal. The ATLAS and CMS collaborations have performed searches for heavy states decaying into $t \bar{t}$ pairs $[151,152]$ but did not specifically address the complicated Higgs situation as only electroweak spin-one resonances, like new neutral gauge bosons or electroweak Kaluza-Klein excitations, were considered. In these two cases, the main production channel is $q \bar{q}$ annihilation and there is no interference with the (colored) QCD $q \bar{q} \rightarrow t \bar{t}$ background and the resonance signal simply appears as a peak in the invariant mass distribution of the $t \bar{t}$ pair.

A full and realistic Monte-Carlo simulation of the $g g \rightarrow H / A \rightarrow t \bar{t}$ process including the effects of the interference and taking into account reconstruction and detector aspects is beyond the scope of this paper, and will be postponed to a future publication [153]. Here, 
we will simply make a very crude estimate of the sensitivity that can be achieved in this channel, relying on previous ATLAS [151] and CMS [152] analyses performed at $\sqrt{s}=8$ $\mathrm{TeV}$ c.m. energy in the spin-one resonance context mentioned above. We will naively consider the number of signal and background events, applying very simple kinematical cuts and ignoring the complicated interference effects, and delineate the area in the $\left[\tan \beta, M_{A}\right]$ $h$ MSSM parameter space in which one has $N_{\text {signal }} / \sqrt{N_{\mathrm{bkg}}} \geq s$. The significance $s=5$ would correspond to a $5 \sigma$ observation of the Higgs signal while $s=2$ would be a first hint of the new effect; in the absence of any effect, $s=2$ would correspond to the $95 \% \mathrm{CL}$ exclusion limit of the phenomenon. To further simplify our analysis, we will assume that the two heavy $A$ and $H$ states are mass degenerate so that the signal rate is simply the sum of the $A$ and $H$ production cross section times the respective branching ratios in their decays into $t \bar{t}$ pairs (which, as we have already seen, is a good approximation).

The main ingredients of the analysis are as follows. The normalization of the Higgs signal has been obtained using the programs HIGLU for the production cross sections and HDECAY for the decay branching ratios. The total cross section of the SM background (which will serve as a normalization) has been obtained using the program Top++ [154]. For the input $m_{t}=173.2 \mathrm{GeV}$ one obtains for the background rate at the first stage of the LHC with $\sqrt{s}=8 \mathrm{TeV}$

$$
\sigma_{\text {tot }}^{\mathrm{QCD}}(p p \rightarrow t \bar{t})=247.7_{-8.5}^{+6.3+11.5}+{ }_{-1.5} \mathrm{pb} \text { at } \sqrt{s}=8 \mathrm{TeV}
$$

when the renormalisation and factorization scales are fixed to $\mu_{R}=\mu_{F}=m_{t}$. In this equation, the first error is the one due to the scale variation within a factor of two from the central scale, and the second one the $\mathrm{PDF}+\alpha_{s}$ uncertainty. This value for the cross section is obtained at NNLO in QCD including the resummation of next-to-next-to-leading logarithmic (NNLL) soft gluon terms and it turns out that it is only $3 \%$ larger than the value of the cross section when evaluated at NNLO [156]. Note that at $\sqrt{s}=14 \mathrm{TeV}$, using the same approximation and ingredients, one would obtain for the cross section

$$
\sigma_{\text {tot }}^{\mathrm{QCD}}(p p \rightarrow t \bar{t})=966.0_{-33.9}^{+22.7}{ }_{-40.5}^{+40.5} \mathrm{pb} \text { at } \sqrt{s}=14 \mathrm{TeV} .
$$

Using the program MadGraph5 [157], we have generated the signal and background cross sections for the process $p p \rightarrow t \bar{t}$. The differential cross section as a function of the invariant mass of the $t \bar{t}$ system, $d \sigma / d m_{t t}$, is shown at $\sqrt{s}=8 \mathrm{TeV}$ in the upper part of figure 16 where mass bins of $10 \mathrm{GeV}$ have been assumed. We overlay on the continuum QCD background distribution (in black solid line), the distributions for the $A$ signal only (the colored lines) with $\tan \beta=1$ and three possible mass values, $M_{A}=400,600$ and $800 \mathrm{GeV}$. In order to see the signals in the figure, we have multiplied the distributions by a factor of 5, 50 and 300, respectively.

In order to enhance the significance $s$, one could apply very basics kinematical cuts that suppress the background while leaving the signal almost unaffected. In the left and right-hand sides of figure 16, we show two distributions (as we are interested in the shapes only, the distributions have not been re-weighted with the correct $K$-factors etc. and the integrated areas thus correspond to the Monte-Carlo cross sections). The first one is 

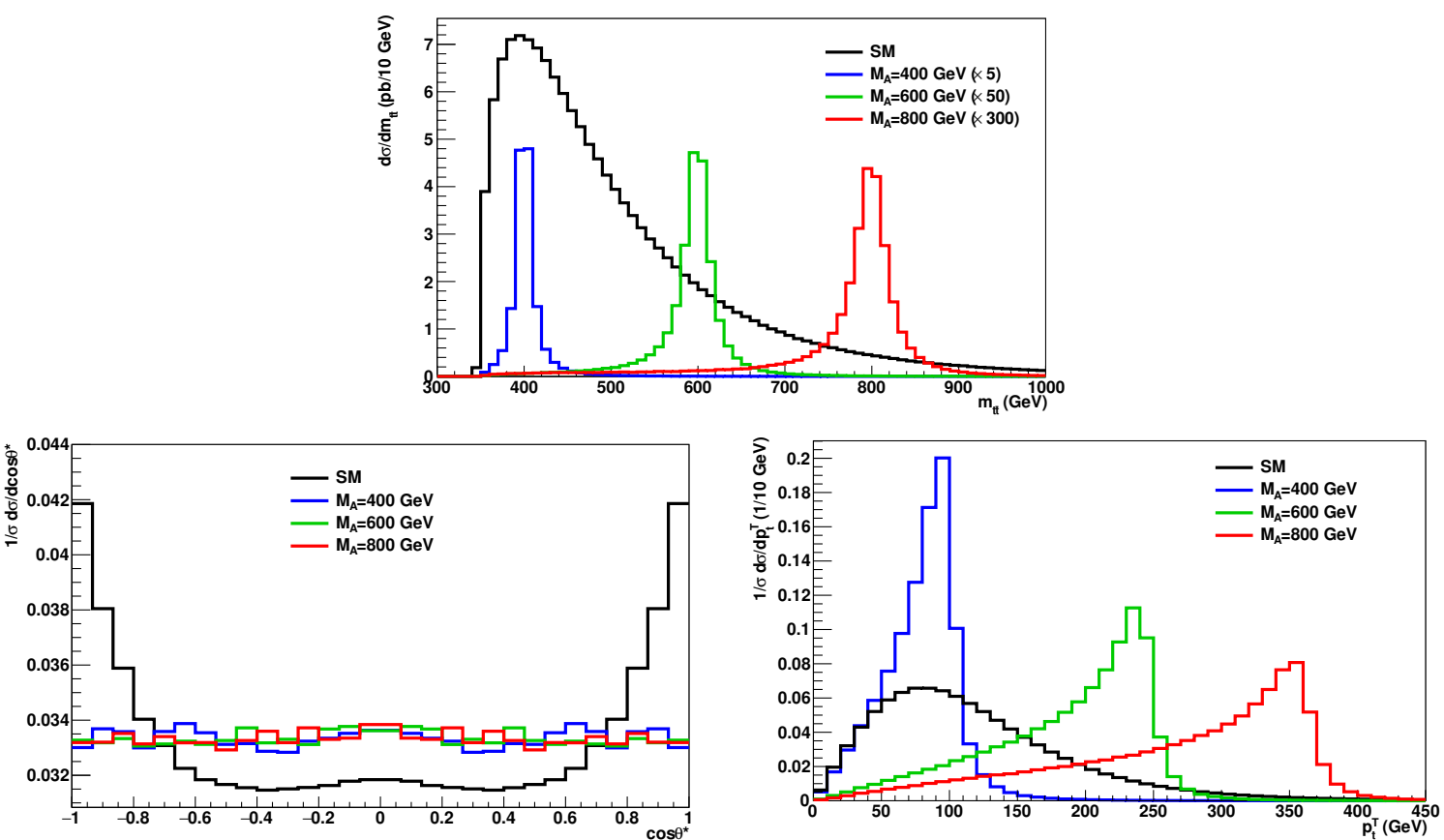

Figure 16. The $p p \rightarrow t \bar{t}$ signal and background at $\sqrt{s}=14 \mathrm{TeV}$ for $M_{A}=400,600$ and $800 \mathrm{GeV}$ and $\tan \beta=1$ : the invariant $m_{t t}$ (top), $\cos \theta^{*}$ (bottom left) and $p_{T}^{t}$ (bottom right) distributions.

$1 / \sigma \times d \sigma / d \cos \theta *$ where $\theta *$ the helicity angle between the off-shell Higgs boson boosted back into the top quark pair rest frame and the top quark pair direction (left). As can be seen, while the signal distribution is almost flat, the background is peaked in the forward and backward directions; a cut $|\cos \theta *| \leq 0.8$ for instance would remove a large sample of background events. A second distribution per $10 \mathrm{GeV}$ bin is in terms of the transverse momentum of the top quarks, $1 / \sigma \times d \sigma / d p_{T}$ (right). They show a characteristic behavior for the signal events, with a pronounced peak and then a sharp drop. One grossly estimates that, for the mass value $M_{A}=800 \mathrm{GeV}$ for instance, a cut on the $p_{T}^{t}$ distribution could allow to suppress the background by a factor of $\approx 6$.

Assuming that when applying all kinematical cuts, one could suppress the $t \bar{t}$ QCD background by an order of magnitude without significantly altering the Higgs signal, we delineate in figure 17 the regions of the $\left[\tan \beta, M_{A}\right]$ plane in which one would expect $N_{\text {signal }} / \sqrt{N_{\mathrm{bkg}}} \geq 2,3,4,5$. For the previous LHC run with $\sqrt{s}=8 \mathrm{TeV}$ and $25 \mathrm{fb}^{-1}$ data (left), one observes that a two $2 \sigma$ "evidence" for a new resonance, or a 95\%CL exclusion of the relevant point of the MSSM parameter space in the absence of any effect, can be achieved for $\tan \beta \approx 2.5$ if $M_{A} \approx 350 \mathrm{GeV}$ and $\tan \beta \approx 1$ when $M_{A} \approx 550 \mathrm{GeV}$. A $5 \sigma$ discovery could be achieved in this case in a much smaller area of the parameter space, $\tan \beta \approx 1.5-1$ and $M_{A} \approx 350-450 \mathrm{GeV}$. The situation could be vastly improved at the next LHC run with $\sqrt{s}=14 \mathrm{TeV}$ and $300 \mathrm{fb}^{-1}$ data (right) as one could be sensitive to $\tan \beta$ values $\tan \beta \approx 7$ for $M_{A} \approx 350 \mathrm{GeV}$ and mass values $M_{A} \approx 1 \mathrm{TeV}$ for $\tan \beta \approx 1$.

The $2 \sigma$ sensitivity in the $\left[\tan \beta, M_{A}\right]$ plane when the $H / A \rightarrow t \bar{t}$ reach (using the the assumptions above) is superimposed to the sensitivity in all the fermionic and bosonic 

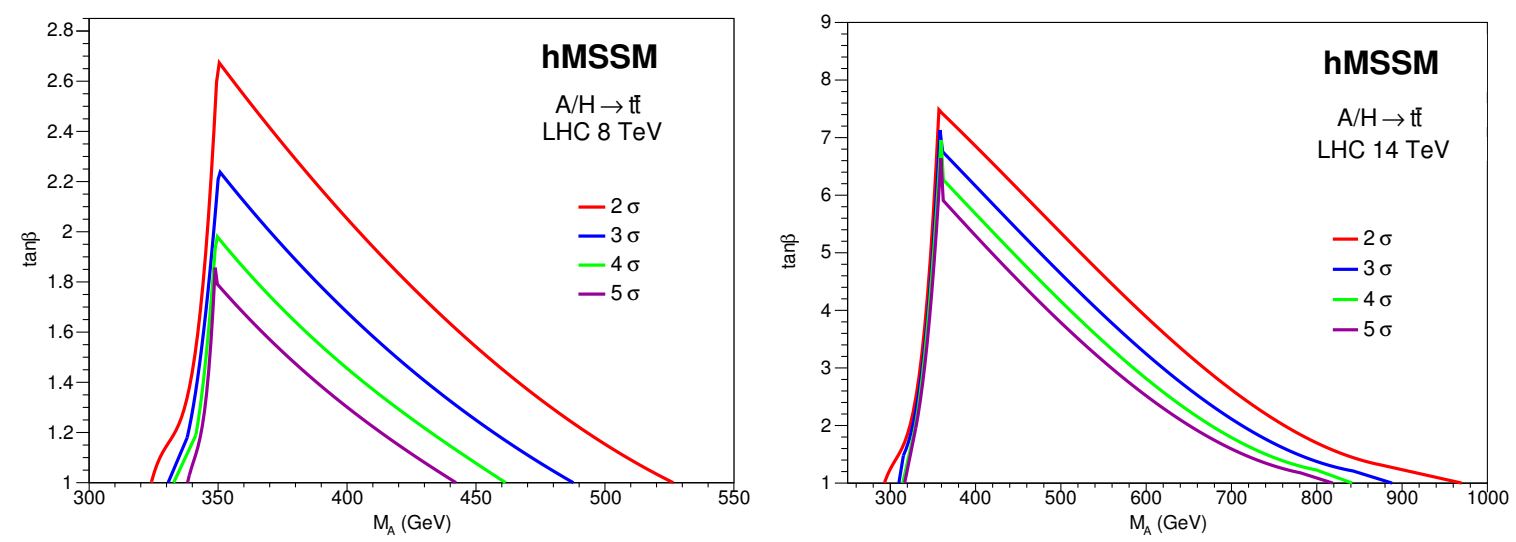

Figure 17. Sensitivity in the $g g \rightarrow H / A \rightarrow t t$ channel in the $\left[\tan \beta, M_{A}\right]$ plane of the $h \mathrm{MSSM}$ at the $2,3,4,5 \sigma$ level $\sqrt{s}=8 \mathrm{TeV}$ and $25 \mathrm{fb}^{-1}$ (left) and $\sqrt{s}=14 \mathrm{TeV}$ and $300 \mathrm{fb}^{-1}$ (right).

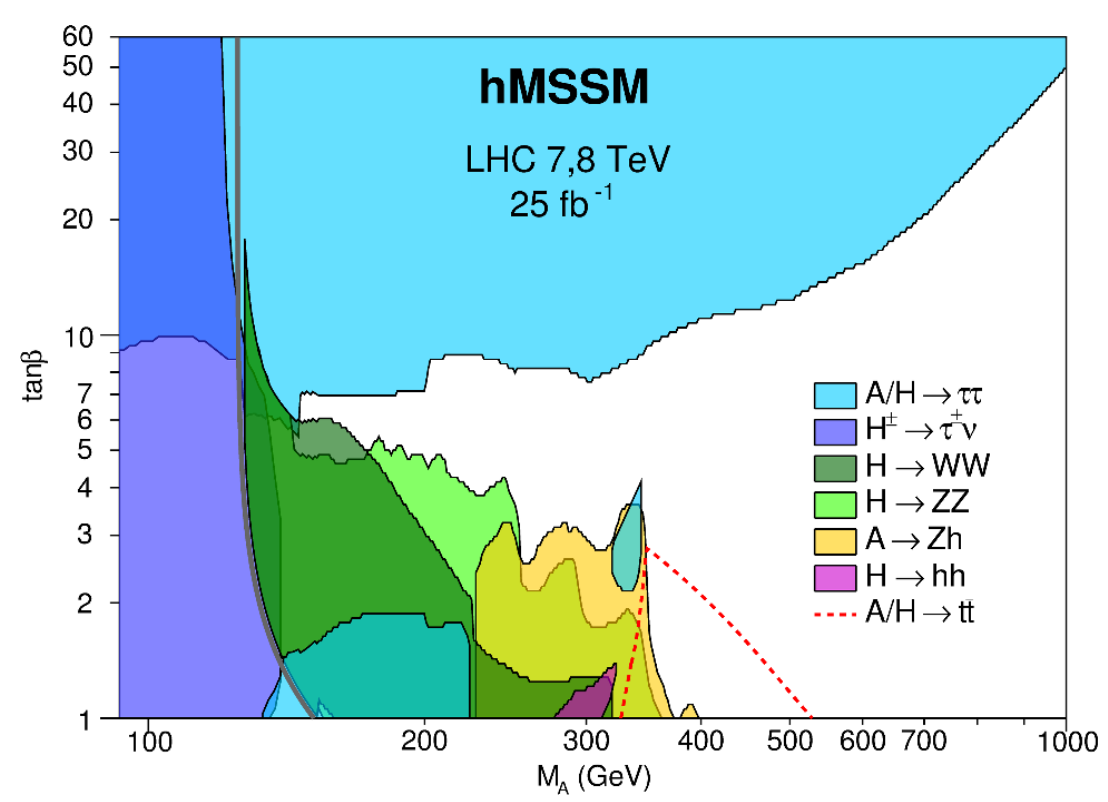

Figure 18. Expectations for the $2 \sigma$ sensitivity in the $h \mathrm{MSSM}\left[\tan \beta, M_{A}\right]$ plane when the searches for the $A / H / H^{ \pm}$states in all channels, including the $g g \rightarrow H / A \rightarrow t t$ process, are combined at the LHC with $\sqrt{s}=8 \mathrm{TeV}$ and $25 \mathrm{fb}^{-1}$ data.

channels discussed previously is displayed in figures 18 and 19 for, respectively, the previous and the next LHC phases. As can be seen, a vast improvement in the sensitivity is expected if the $H / A \rightarrow t \bar{t}$ channel is included, in particular at the forthcoming LHC run with $\sqrt{s}=14 \mathrm{TeV}$ and $300 \mathrm{fb}^{-1}$ data. The improvement is even more impressive at the high-luminosity LHC option, when the luminosity is increased to $3000 \mathrm{fb}^{-1}$; see figure 20 . In this case, almost the entire $h \mathrm{MSSM}$ parameter space, up to $M_{A}$ values close to $\approx 1 \mathrm{TeV}$, can be probed. 


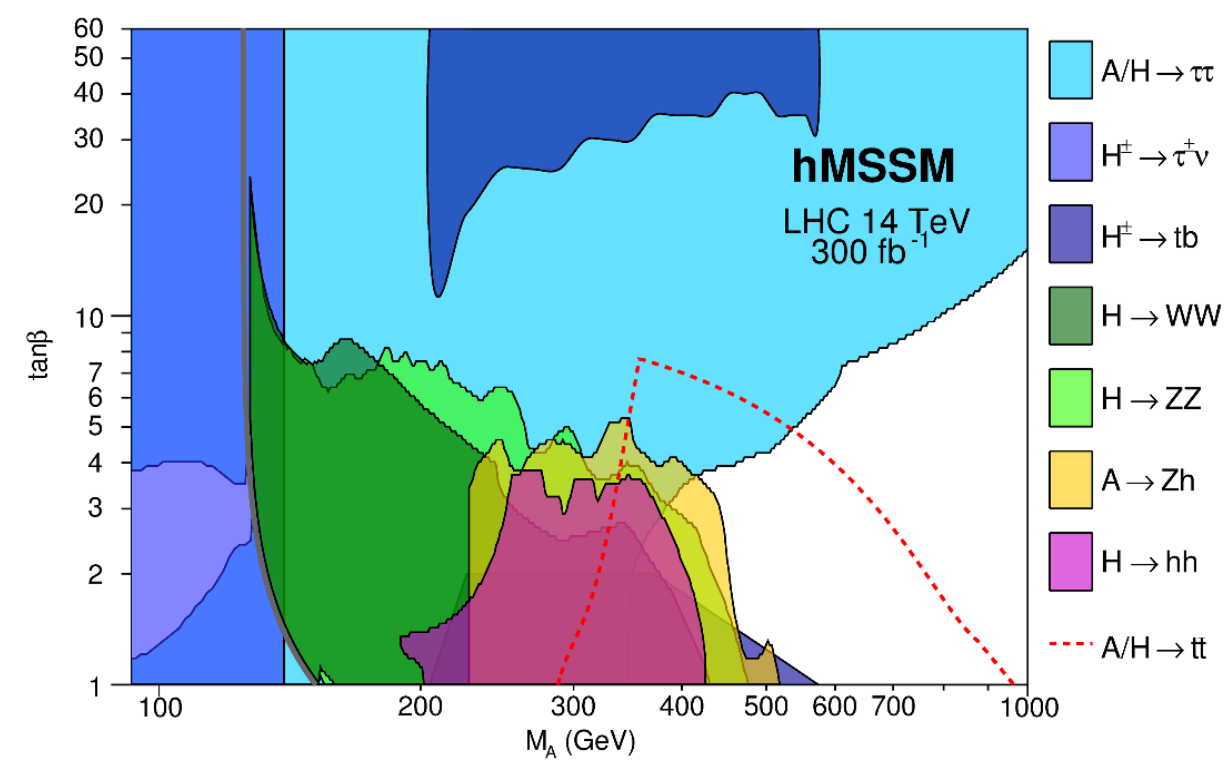

Figure 19. The same as in figure 18 but at the LHC with $\sqrt{s}=14 \mathrm{TeV}$ and $300 \mathrm{fb}^{-1}$ data.

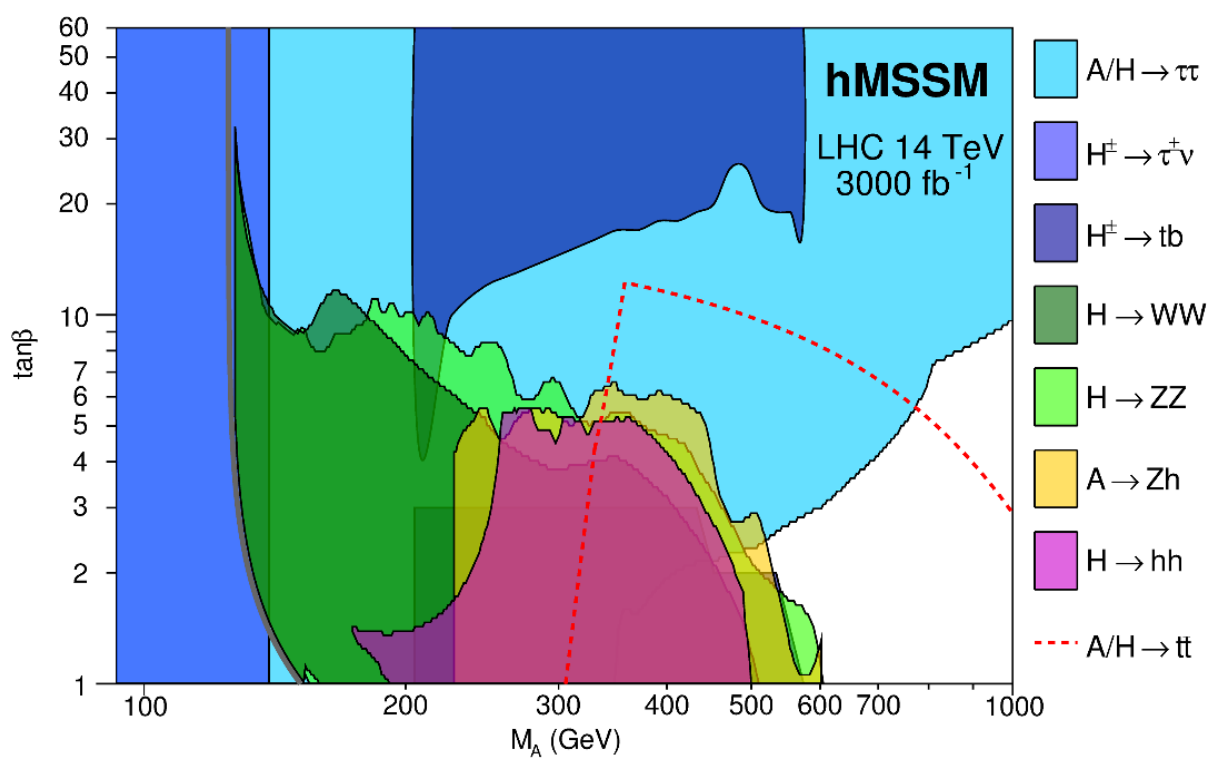

Figure 20. The same as figure 18 but at the high-luminosity LHC option with $3000 \mathrm{fb}^{-1}$ data.

\section{Conclusions}

In this paper, we have addressed the issue of covering the entire parameter space of the MSSM Higgs sector at the LHC by considering the search of the heavier $H, A$ and $H^{ \pm}$ states that are predicted in the model, in addition to the already observed lightest $h$ boson. These searches should not only be restricted to the channels that have been considered so far by the ATLAS and CMS collaborations, namely those with a surplus of $\tau \nu$ events and those with high mass resonances decaying into $\tau$ lepton pairs, which would signal the 
presence of new contributions from the $t \rightarrow b H^{+} \rightarrow b \tau \nu$ and $p p \rightarrow H / A \rightarrow \tau \tau$ processes, which are mainly relevant for the high $\tan \beta$ region of the MSSM Higgs sector. Search for heavier Higgs bosons should also be conducted in channels that are more appropriate for the probing of the low $\tan \beta$ region and which, until now, have been overlooked.

We have first discussed and refined the hMSSM approach introduced in ref. [26] in which the dominant radiative corrections to the MSSM Higgs sector, that introduce a dependence on numerous SUSY parameters, are traded against the measured mass $M_{h}=$ $125 \mathrm{GeV}$ of the Higgs boson which was observed at the LHC, thus allowing to describe again the entire Higgs sector of the model with only two input parameters. This simple, economical and "model independent" approach permits to reopen the low $\tan \beta$ region, at the expense of considering the possibility that the scale of SUSY-breaking is extremely high, $M_{S} \gg 1 \mathrm{TeV}$, and that the model is severely fine-tuned. The $h \mathrm{MSSM}$ is expected to be viable down to values $\tan \beta \approx 2$ and, for higher $\tan \beta$ values, reproduces to a very good approximation the standard results of the MSSM Higgs sector. This is particularly true if the higgsino mass parameter is much smaller than the SUSY-breaking scale, $\mu \ll M_{S}$, an assumption that is natural at low $\tan \beta$ values which imply a very high SUSY-breaking scale. Thus, searches for new signals in the MSSM Higgs sector can be performed in the entire $\left[\tan \beta, M_{A}\right]$ parameter space, in a reliable way for $\tan \beta \gtrsim 2$. Nevertheless, in an effective approach, one can eventually extrapolate to values of $\tan \beta$ very close to unity, despite of the fact that the scale $M_{S}$ required to reach this value is so high that its renders the model not only too fine-tuned but also potentially inconsistent.

We have then analyzed the production and decay modes of the $H, A$ and $H^{ \pm}$particles at the LHC, with a special attention to the low $\tan \beta$ region in which the top quark plays a prominent role, as its couplings to the Higgs bosons are not strongly suppressed compared to the SM case. We have first shown that the searches that are presently conducted by ATLAS and CMS can also be relevant at low $\tan \beta$. This is for instance the case of the $p p \rightarrow A \rightarrow \tau \tau$ and $p p \rightarrow t b H^{+} \rightarrow t b t b$ processes at low to moderate $M_{A}$ values. We have then shown that search channels such as $H \rightarrow W W, Z Z, h h$ and $A \rightarrow h Z$, when interpreted in the context of the $h \mathrm{MSSM}$, can also probe the low $\tan \beta$ and not too high $M_{A}$ regions. In fact, already with the $25 \mathrm{fb}^{-1}$ data collected at energies up to $\sqrt{s}=8 \mathrm{TeV}$, the ATLAS and CMS collaborations exclude the possibility $\tan \beta \lesssim 4(2)$ up to pseudoscalar Higgs masses of $M_{A} \approx 250(350) \mathrm{GeV}$. At the upcoming stage of the LHC, with an expected energy and luminosity of $\sqrt{s}=14 \mathrm{TeV}$ and $\mathcal{L}=300 \mathrm{fb}^{-1}$, the entire parameter space i.e. for any value of $\tan \beta$ could be probed up to $M_{A} \approx 400 \mathrm{GeV}$, when combining the searches in the usual fermionic channels and in the additional bosonic channels discussed here.

An important message conveyed by the present paper is that, in order to fill or close the gap in the MSSM $\left[\tan \beta, M_{A}\right.$ ] plane left by the fermionic and bosonic searches mentioned above, one should definitely consider the $p p \rightarrow H / A \rightarrow t \bar{t}$ process. Indeed, at low $\tan \beta$ and for Higgs masses above the $t \bar{t}$ kinematical threshold, the decays $H / A \rightarrow t \bar{t}$ become the dominant ones, suppressing the rates for the other decay channels to a very low if not negligible level. On the other hand, the $g g \rightarrow H / A$ production mode has a still significant cross section as the top quark that generates this loop process has substantial couplings to the $H / A$ states at sufficiently low $\tan \beta$ values. This is not a very easy search channel 
in view of the formidable $p p \rightarrow t \bar{t}$ QCD background. Nevertheless, it exhibits very special and interesting features such as an interference with the QCD background that leads to a rather involved peak-dip structure of the signal.

We have not performed a detailed and realistic study of this process but attempted to roughly quantify the observation of a signal at the LHC, relying on present ATLAS and CMS analyses in searches for heavy (non Higgs) resonances decaying into top quark pairs at $8 \mathrm{TeV}$ center of mass energies, and discussed its possible implications. It appears that the channel $g g \rightarrow H / A \rightarrow t \bar{t}$, would be capable of covering partly the area at low $\tan \beta$ and high $M_{A}$, hence allowing for a full coverage of the $\left[\tan \beta, M_{A}\right]$ plane of the MSSM up to Higgs masses $M_{A} \approx 600 \mathrm{GeV}$ with $300 \mathrm{fb}^{-1}$ data at $\sqrt{s}=14 \mathrm{TeV}$. At the high luminosity option of the LHC with $3000 \mathrm{fb}^{-1}$ data, one could reach a full coverage of the MSSM parameter space for pseudoscalar masses closer to $M_{A} \approx 1 \mathrm{TeV}$.

More refined analyses are required in order to firmly establish the viability of the various processes discussed here, in particular the $H / A \rightarrow t \bar{t}$ channel. In view of the important role that it could play in the probing of the MSSM parameter space, the latter process is worth investigating in a more realistic way, including the interference between the Higgs signal and the QCD background. This is what we plan to do in a forthcoming publication [153].

\section{Acknowledgments}

Discussions with the LHC Higgs cross section working group on the $h$ MSSM, in particular, S. Heinemeyer, G. Lee, M. Muhlleitner, S. Nikitenko, N. Rompotis, P. Slavich, M. Spira, C. Wagner and G. Weiglein, are greatfully acknowledged. AD is supported by the ERC advanced grant Higgs@LHC and JQ by the STFC Grant ST/J002798/1.

Open Access. This article is distributed under the terms of the Creative Commons Attribution License (CC-BY 4.0), which permits any use, distribution and reproduction in any medium, provided the original author(s) and source are credited.

\section{References}

[1] M. Drees, R. Godbole and P. Roy, Theory and phenomenology of sparticles, World Scientific, (2005).

[2] H. Baer and X. Tata, Weak scale Supersymmetry: from superfields to scattering events, Cambridge University Press, (2006).

[3] S.P. Martin, A supersymmetry primer, Adv. Ser. Direct. High Energy Phys. 21 (2010) 1 [hep-ph/9709356] [INSPIRE].

[4] ATLAS collaboration, Observation of a new particle in the search for the Standard Model Higgs boson with the ATLAS detector at the LHC, Phys. Lett. B 716 (2012) 1 [arXiv:1207.7214] [INSPIRE].

[5] CMS collaboration, Observation of a new boson at a mass of $125 \mathrm{GeV}$ with the CMS experiment at the LHC, Phys. Lett. B 716 (2012) 30 [arXiv:1207.7235] [InSPIRE]. 
[6] J. Gunion, H. Haber, G. Kane and S. Dawson, The Higgs Hunter's Guide, Reading, (1990).

[7] M. Spira, QCD effects in Higgs physics, Fortsch. Phys. 46 (1998) 203 [hep-ph/9705337] [INSPIRE].

[8] A. Djouadi, The anatomy of electro-weak symmetry breaking. I: The Higgs boson in the standard model, Phys. Rept. 457 (2008) 1 [hep-ph/0503172] [INSPIRE].

[9] M. Carena and H.E. Haber, Higgs boson theory and phenomenology, Prog. Part. Nucl. Phys. 50 (2003) 63 [hep-ph/0208209] [INSPIRE].

[10] A. Djouadi, The anatomy of electro-weak symmetry breaking. II. The Higgs bosons in the minimal supersymmetric model, Phys. Rept. 459 (2008) 1 [hep-ph/0503173] [INSPIRE].

[11] ATLAS collaboration, Measurements of Higgs boson production and couplings in diboson final states with the ATLAS detector at the LHC, Phys. Lett. B 726 (2013) 88 [arXiv: 1307.1427] [INSPIRE].

[12] ATLAS collaboration, Updated coupling measurements of the Higgs boson with the ATLAS detector using up to $25 \mathrm{fb}^{-1}$ of proton-proton collision data, ATLAS-CONF-2014-009 (2014).

[13] CMS collaboration, Precise determination of the mass of the Higgs boson and tests of compatibility of its couplings with the standard model predictions using proton collisions at 7 and $8 \mathrm{TeV}$, Eur. Phys. J. C 75 (2015) 212 [CMS-PAS-HIG-14-009] [arXiv:1412.8662] [INSPIRE].

[14] Y. Okada, M. Yamaguchi and T. Yanagida, Upper bound of the lightest Higgs boson mass in the minimal supersymmetric standard model, Prog. Theor. Phys. 85 (1991) 1 [INSPIRE].

[15] J.R. Ellis, G. Ridolfi and F. Zwirner, Radiative corrections to the masses of supersymmetric Higgs bosons, Phys. Lett. B 257 (1991) 83 [INSPIRE].

[16] H.E. Haber and R. Hempfling, Can the mass of the lightest Higgs boson of the minimal supersymmetric model be larger than $m_{Z}$ ?, Phys. Rev. Lett. 66 (1991) 1815 [INSPIRE].

[17] M. Carena, J.R. Espinosa, M. Quirós and C.E.M. Wagner, Analytical expressions for radiatively corrected Higgs masses and couplings in the MSSM, Phys. Lett. B 355 (1995) 209 [hep-ph/9504316] [INSPIRE].

[18] H.E. Haber, R. Hempfling and A.H. Hoang, Approximating the radiatively corrected Higgs mass in the minimal supersymmetric model, Z. Phys. C 75 (1997) 539 [hep-ph/9609331] [INSPIRE].

[19] S. Heinemeyer, W. Hollik and G. Weiglein, QCD corrections to the masses of the neutral CP-even Higgs bosons in the MSSM, Phys. Rev. D 58 (1998) 091701 [hep-ph/9803277] [INSPIRE].

[20] S. Heinemeyer, W. Hollik and G. Weiglein, The masses of the neutral CP-even Higgs bosons in the MSSM: Accurate analysis at the two loop level, Eur. Phys. J. C 9 (1999) 343 [hep-ph/9812472] [INSPIRE].

[21] G. Degrassi, P. Slavich and F. Zwirner, On the neutral Higgs boson masses in the MSSM for arbitrary stop mixing, Nucl. Phys. B 611 (2001) 403 [hep-ph/0105096] [INSPIRE].

[22] A. Brignole, G. Degrassi, P. Slavich and F. Zwirner, On the $\mathcal{O}\left(\alpha_{t}^{2}\right)$ two loop corrections to the neutral Higgs boson masses in the MSSM, Nucl. Phys. B 631 (2002) 195 [hep-ph/0112177] [INSPIRE]. 
[23] A. Brignole, G. Degrassi, P. Slavich and F. Zwirner, On the two loop sbottom corrections to the neutral Higgs boson masses in the MSSM, Nucl. Phys. B 643 (2002) 79 [hep-ph/0206101] [INSPIRE].

[24] S.P. Martin, Three-loop corrections to the lightest Higgs scalar boson mass in supersymmetry, Phys. Rev. D 75 (2007) 055005 [hep-ph/0701051] [INSPIRE].

[25] P. Kant, R.V. Harlander, L. Mihaila and M. Steinhauser, Light MSSM Higgs boson mass to three-loop accuracy, JHEP 08 (2010) 104 [arXiv: 1005.5709] [INSPIRE].

[26] A. Djouadi, L. Maiani, G. Moreau, A. Polosa, J. Quevillon and V. Riquer, The post-Higgs MSSM scenario: Habemus MSSM?, Eur. Phys. J. C 73 (2013) 2650 [arXiv:1307.5205] [INSPIRE].

[27] L. Maiani, A.D. Polosa and V. Riquer, Probing Minimal Supersymmetry at the LHC with the Higgs Boson Masses, New J. Phys. 14 (2012) 073029 [arXiv: 1202.5998] [InSPIRE].

[28] L. Maiani, A.D. Polosa and V. Riquer, Heavier Higgs Particles: Indications from Minimal Supersymmetry, Phys. Lett. B 718 (2012) 465 [arXiv:1209.4816] [INSPIRE].

[29] L. Maiani, A.D. Polosa and V. Riquer, Bounds to the Higgs Sector Masses in Minimal Supersymmetry from LHC Data, Phys. Lett. B 724 (2013) 274 [arXiv:1305.2172] [INSPIRE].

[30] A. Djouadi and J. Quevillon, The MSSM Higgs sector at a high $M_{S U S Y}$ : reopening the low $\tan \beta$ regime and heavy Higgs searches, JHEP 10 (2013) 028 [arXiv: 1304.1787] [INSPIRE].

[31] A. Djouadi and G. Moreau, The couplings of the Higgs boson and its CP properties from fits of the signal strengths and their ratios at the $7+8$ TeV LHC, Eur. Phys. J. C 73 (2013) 2512 [arXiv: 1303.6591] [INSPIRE].

[32] M. Carena, H.E. Haber, I. Low, N.R. Shah and C.E.M. Wagner, Complementarity between nonstandard Higgs boson searches and precision Higgs boson measurements in the MSSM, Phys. Rev. D 91 (2015) 035003 [arXiv:1410.4969] [INSPIRE].

[33] ATLAS collaboration, Search for charged Higgs bosons in the $\tau+$ jets final state with $p p$ collision data recorded at $\sqrt{s}=8 \mathrm{TeV}$ with the ATLAS experiment, ATLAS-CONF-2013-090 (2013).

[34] CMS collaboration, Search for charged Higgs bosons with the $H^{+} \rightarrow \tau^{+} \nu_{\tau}$ decay channel in the fully hadronic final state at $\sqrt{s}=8 \mathrm{TeV}$, CMS-HIG-14-020.

[35] ATLAS collaboration, Search for neutral Higgs bosons of the minimal supersymmetric standard model in pp collisions at $\sqrt{s}=8 \mathrm{TeV}$ with the ATLAS detector, JHEP 11 (2014) 056 [arXiv: 1409.6064] [INSPIRE].

[36] CMS collaboration, Search for neutral MSSM Higgs bosons decaying to a pair of tau leptons in pp collisions, JHEP 10 (2014) 160 [CMS-PAS-HIG-13-021] [arXiv:1408.3316] [INSPIRE].

[37] Particle Data Group collaboration, K. Olive et al., Review of Particle Physics, Chin. Phys. C 38 (2014) 090001.

[38] LEP Working Group for Higgs boson searches, ALEPH, DELPHI, L3, OPAL collaborations, R. Barate et al., Search for the standard model Higgs boson at LEP, Phys. Lett. B 565 (2003) 61 [hep-ex/0306033] [INSPIRE].

[39] M. Carena, S. Heinemeyer, C.E.M. Wagner and G. Weiglein, Suggestions for benchmark scenarios for MSSM Higgs boson searches at hadron colliders, Eur. Phys. J. C 26 (2003) 601 [hep-ph/0202167] [INSPIRE]. 
[40] M. Carena, S. Heinemeyer, O. Stål, C.E.M. Wagner and G. Weiglein, MSSM Higgs Boson Searches at the LHC: Benchmark Scenarios after the Discovery of a Higgs-like Particle, Eur. Phys. J. C 73 (2013) 2552 [arXiv:1302.7033] [INSPIRE].

[41] G. Degrassi, S. Heinemeyer, W. Hollik, P. Slavich and G. Weiglein, Towards high precision predictions for the MSSM Higgs sector, Eur. Phys. J. C 28 (2003) 133 [hep-ph/0212020] [INSPIRE].

[42] B.C. Allanach, A. Djouadi, J.L. Kneur, W. Porod and P. Slavich, Precise determination of the neutral Higgs boson masses in the MSSM, JHEP 09 (2004) 044 [hep-ph/0406166] [INSPIRE].

[43] S. Heinemeyer, W. Hollik and G. Weiglein, Electroweak precision observables in the minimal supersymmetric standard model, Phys. Rept. 425 (2006) 265 [hep-ph/0412214] [INSPIRE].

[44] S. Heinemeyer, MSSM Higgs physics at higher orders, Int. J. Mod. Phys. A 21 (2006) 2659 [hep-ph/0407244] [INSPIRE].

[45] CMS collaboration, Measurement of Higgs boson production and properties in the $W W$ decay channel with leptonic final states, JHEP 01 (2014) 096 [arXiv:1312.1129] [INSPIRE].

[46] CMS collaboration, Measurement of the properties of a Higgs boson in the four-lepton final state, Phys. Rev. D 89 (2014) 092007 [arXiv:1312.5353] [INSPIRE].

[47] ATLAS collaboration, Search For Higgs Boson Pair Production in the $\gamma \gamma b \bar{b}$ Final State using pp Collision Data at $\sqrt{s}=8$ TeV from the ATLAS Detector, Phys. Rev. Lett. 114 (2015) 081802 [arXiv: 1406.5053] [INSPIRE].

[48] CMS collaboration, Search for resonant HH production in 2gamma+2b channel, CMS-PAS-HIG-13-032.

[49] CMS collaboration, Search for a pseudoscalar boson $A$ decaying into a $Z$ and an $h$ boson in the llbb final state, CMS-PAS-HIG-14-011.

[50] A. Arbey, M. Battaglia and F. Mahmoudi, Supersymmetric Heavy Higgs Bosons at the LHC, Phys. Rev. D 88 (2013) 015007 [arXiv:1303.7450] [InSPIRE].

[51] P. Bechtle, S. Heinemeyer, O. Stål, T. Stefaniak and G. Weiglein, HiggsSignals: Confronting arbitrary Higgs sectors with measurements at the Tevatron and the LHC, Eur. Phys. J. C 74 (2014) 2711 [arXiv:1305.1933] [inSPIRE].

[52] N. Craig, J. Galloway and S. Thomas, Searching for Signs of the Second Higgs Doublet, arXiv:1305.2424 [INSPIRE].

[53] N.D. Christensen, T. Han and S. Su, MSSM Higgs Bosons at The LHC, Phys. Rev. D 85 (2012) 115018 [arXiv:1203.3207] [InSPIRE].

[54] E. Arganda, J.L. Diaz-Cruz and A. Szynkman, Slim SUSY, Phys. Lett. B 722 (2013) 100 [arXiv: 1301.0708] [INSPIRE].

[55] E. Arganda, J.L. Diaz-Cruz and A. Szynkman, Decays of $H^{0} / A^{0}$ in supersymmetric scenarios with heavy sfermions, Eur. Phys. J. C 73 (2013) 2384 [arXiv:1211.0163] [INSPIRE].

[56] P.S.B. Dev and A. Pilaftsis, Maximally Symmetric Two Higgs Doublet Model with Natural Standard Model Alignment, JHEP 12 (2014) 024 [arXiv:1408.3405] [INSPIRE].

[57] P. Slavich, Low-tan $\beta$ scenario vs. hMSSM vs. effective THDM, talk given at The 8th workshop of LHC Higgs cross section working group, CERN, 22-24 January 2015, https://indico.cern.ch/event/331452/. 
[58] P. Draper, G. Lee and C.E.M. Wagner, Precise estimates of the Higgs mass in heavy supersymmetry, Phys. Rev. D 89 (2014) 055023 [arXiv: 1312.5743] [INSPIRE].

[59] K. Cheung, R. Huo, J.S. Lee and Y.-L. Sming Tsai, Dark Matter in Split SUSY with Intermediate Higgses, JHEP 04 (2015) 151 [arXiv: 1411.7329] [INSPIRE].

[60] G. Lee et al., in preparation.

[61] M. Frank et al., Charged Higgs Boson Mass of the MSSM in the Feynman Diagrammatic Approach, Phys. Rev. D 88 (2013) 055013 [arXiv:1306.1156] [INSPIRE].

[62] A. Brignole and F. Zwirner, Radiative corrections to the decay $H \rightarrow$ hh in the minimal supersymmetric standard model, Phys. Lett. B 299 (1993) 72 [hep-ph/9210266] [INSPIRE].

[63] S. Heinemeyer and W. Hollik, The decay $h^{0} \rightarrow A^{0} \rightarrow A^{0} A^{0}: A$ complete one loop calculation in the MSSM, Nucl. Phys. B 474 (1996) 32 [hep-ph/9602318] [INSPIRE].

[64] V.D. Barger, M.S. Berger, A.L. Stange and R.J.N. Phillips, Supersymmetric Higgs boson hadroproduction and decays including radiative corrections, Phys. Rev. D 45 (1992) 4128 [INSPIRE].

[65] A. Dobado, M.J. Herrero, W. Hollik and S. Penaranda, Selfinteractions of the lightest MSSM Higgs boson in the large pseudoscalar mass limit, Phys. Rev. D 66 (2002) 095016 [hep-ph/0208014] [INSPIRE].

[66] M. Brucherseifer, R. Gavin and M. Spira, Minimal supersymmetric Higgs boson self-couplings: Two-loop $O\left(\alpha_{t} \alpha_{s}\right)$ corrections, Phys. Rev. D 90 (2014) 117701 [arXiv: 1309.3140] [INSPIRE].

[67] A. Djouadi, J.-L. Kneur and G. Moultaka, SuSpect: A Fortran code for the supersymmetric and Higgs particle spectrum in the MSSM, Comput. Phys. Commun. 176 (2007) 426 [hep-ph/0211331] [INSPIRE].

[68] S. Heinemeyer, W. Hollik and G. Weiglein, FeynHiggs: A program for the calculation of the masses of the neutral CP even Higgs bosons in the MSSM, Comput. Phys. Commun. 124 (2000) 76 [hep-ph/9812320] [INSPIRE].

[69] G.C. Branco et al., Theory and phenomenology of two-Higgs-doublet models, Phys. Rept. 516 (2012) 1 [arXiv:1106.0034] [InSPIRE].

[70] H. Baer, V. Barger and A. Mustafayev, Implications of a $125 \mathrm{GeV}$ Higgs scalar for LHC SUSY and neutralino dark matter searches, Phys. Rev. D 85 (2012) 075010 [arXiv: 1112.3017] [INSPIRE].

[71] P. Draper, P. Meade, M. Reece and D. Shih, Implications of a 125 GeV Higgs for the MSSM and Low-Scale SUSY Breaking, Phys. Rev. D 85 (2012) 095007 [arXiv:1112.3068] [INSPIRE].

[72] O. Buchmueller et al., Higgs and Supersymmetry, Eur. Phys. J. C 72 (2012) 2020 [arXiv: 1112.3564] [INSPIRE].

[73] L.J. Hall, D. Pinner and J.T. Ruderman, A Natural SUSY Higgs Near 126 GeV, JHEP 04 (2012) 131 [arXiv: 1112.2703] [INSPIRE].

[74] S. Heinemeyer, O. Stal and G. Weiglein, Interpreting the LHC Higgs Search Results in the MSSM, Phys. Lett. B 710 (2012) 201 [arXiv:1112.3026] [INSPIRE].

[75] A. Arvanitaki and G. Villadoro, A Non Standard Model Higgs at the LHC as a Sign of Naturalness, JHEP 02 (2012) 144 [arXiv:1112.4835] [INSPIRE]. 
[76] A. Arbey, M. Battaglia, A. Djouadi, F. Mahmoudi and J. Quevillon, Implications of a 125 GeV Higgs for supersymmetric models, Phys. Lett. B 708 (2012) 162 [arXiv:1112.3028] [INSPIRE].

[77] A. Arbey, M. Battaglia, A. Djouadi and F. Mahmoudi, An update on the constraints on the phenomenological MSSM from the new LHC Higgs results, Phys. Lett. B 720 (2013) 153 [arXiv: 1211.4004] [INSPIRE].

[78] A. Arbey, M. Battaglia, A. Djouadi and F. Mahmoudi, The Higgs sector of the phenomenological MSSM in the light of the Higgs boson discovery, JHEP 09 (2012) 107 [arXiv: 1207.1348] [INSPIRE].

[79] A. Djouadi, Implications of the Higgs discovery for the MSSM, Eur. Phys. J. C 74 (2014) 2704 [arXiv: 1311.0720] [INSPIRE].

[80] N. Craig, The State of Supersymmetry after Run I of the LHC, arXiv:1309.0528 [INSPIRE].

[81] N. Bernal, A. Djouadi and P. Slavich, The MSSM with heavy scalars, JHEP 07 (2007) 016 [arXiv:0705.1496] [INSPIRE].

[82] A. Djouadi, J. Kalinowski and M. Spira, HDECAY: A program for Higgs boson decays in the standard model and its supersymmetric extension, Comput. Phys. Commun. 108 (1998) 56 [hep-ph/9704448] [INSPIRE].

[83] A. Djouadi, M.M. Muhlleitner and M. Spira, Decays of supersymmetric particles: The Program SUSY-HIT (SUspect-SdecaY-HDECAY-InTerface), Acta Phys. Polon. B 38 (2007) 635 [hep-ph/0609292] [inSPIRE].

[84] A. Djouadi, J. Kalinowski and P.M. Zerwas, Two and three-body decay modes of SUSY Higgs particles, Z. Phys. C 70 (1996) 435 [hep-ph/9511342] [INSPIRE].

[85] S. Moretti and W.J. Stirling, Contributions of below threshold decays to MSSM Higgs branching ratios, Phys. Lett. B 347 (1995) 291 [hep-ph/9412209] [INSPIRE].

[86] F. Borzumati and A. Djouadi, Lower bounds on charged Higgs bosons from LEP and Tevatron, Phys. Lett. B 549 (2002) 170 [hep-ph/9806301] [InSPIRE].

[87] H.M. Georgi, S.L. Glashow, M.E. Machacek and D.V. Nanopoulos, Higgs Bosons from Two Gluon Annihilation in Proton Proton Collisions, Phys. Rev. Lett. 40 (1978) 692 [InSPIRE].

[88] D.A. Dicus and S. Willenbrock, Higgs Boson Production from Heavy Quark Fusion, Phys. Rev. D 39 (1989) 751 [INSPIRE].

[89] S. Dittmaier, M. Kramer and M. Spira, Higgs radiation off bottom quarks at the Tevatron and the CERN LHC, Phys. Rev. D 70 (2004) 074010 [hep-ph/0309204] [INSPIRE].

[90] S. Dawson, C.B. Jackson, L. Reina and D. Wackeroth, Exclusive Higgs boson production with bottom quarks at hadron colliders, Phys. Rev. D 69 (2004) 074027 [hep-ph/0311067] [INSPIRE].

[91] R.V. Harlander and W.B. Kilgore, Higgs boson production in bottom quark fusion at next-to-next-to leading order, Phys. Rev. D 68 (2003) 013001 [hep-ph/0304035] [INSPIRE].

[92] M. Spira, A. Djouadi, D. Graudenz and P.M. Zerwas, Higgs boson production at the LHC, Nucl. Phys. B 453 (1995) 17 [hep-ph/9504378] [INSPIRE].

[93] R.V. Harlander and W.B. Kilgore, Next-to-next-to-leading order Higgs production at hadron colliders, Phys. Rev. Lett. 88 (2002) 201801 [hep-ph/0201206] [INSPIRE]. 
[94] C. Anastasiou and K. Melnikov, Higgs boson production at hadron colliders in NNLO QCD, Nucl. Phys. B 646 (2002) 220 [hep-ph/0207004] [INSPIRE].

[95] V. Ravindran, J. Smith and W.L. van Neerven, NNLO corrections to the total cross-section for Higgs boson production in hadron hadron collisions, Nucl. Phys. B 665 (2003) 325 [hep-ph/0302135] [INSPIRE].

[96] R.V. Harlander and W.B. Kilgore, Production of a pseudoscalar Higgs boson at hadron colliders at next-to-next-to leading order, JHEP 10 (2002) 017 [hep-ph/0208096] [INSPIRE].

[97] LHC Higgs Cross section Working Group collaboration, S. Dittmaier et al., Handbook of LHC Higgs Cross sections: 1. Inclusive Observables, arXiv:1101.0593 [INSPIRE].

[98] J. Baglio and A. Djouadi, Higgs production at the LHC, JHEP 03 (2011) 055 [arXiv: 1012.0530] [INSPIRE].

[99] J. Baglio and A. Djouadi, Revisiting the constraints on the Supersymmetric Higgs sector at the Tevatron, Phys. Lett. B 699 (2011) 372 [arXiv:1012.2748] [InSPIRE].

[100] J. Baglio, A. Djouadi and R.M. Godbole, The apparent excess in the Higgs to di-photon rate at the LHC: New Physics or QCD uncertainties?, Phys. Lett. B 716 (2012) 203 [arXiv: 1207.1451] [INSPIRE].

[101] A.D. Martin, W.J. Stirling, R.S. Thorne and G. Watt, Parton distributions for the LHC, Eur. Phys. J. C 63 (2009) 189 [arXiv:0901.0002] [inSPIRE].

[102] R.V. Harlander, S. Liebler and H. Mantler, SusHi: A program for the calculation of Higgs production in gluon fusion and bottom-quark annihilation in the Standard Model and the MSSM, Comput. Phys. Commun. 184 (2013) 1605 [arXiv:1212.3249] [INSPIRE].

[103] M. Spira, http://tiger.web.psi.ch.

[104] M. Spira, HIGLU: A program for the calculation of the total Higgs production cross-section at hadron colliders via gluon fusion including QCD corrections, hep-ph/9510347 [INSPIRE].

[105] F. Borzumati, J.L. Kneur and N. Polonsky, Charged-Higgs- and R-parity violating slepton-strahlung at hadron colliders, Phys. Rev. D 60 (1999) 115011.

[106] D.J. Miller, S. Moretti, D.P. Roy and W.J. Stirling, Detecting heavy charged Higgs bosons at the CERN LHC with four b quark tags, Phys. Rev. D 61 (2000) 055011 [hep-ph/9906230] [INSPIRE].

[107] T. Plehn, Charged Higgs boson production in bottom gluon fusion, Phys. Rev. D 67 (2003) 014018 [hep-ph/0206121] [INSPIRE].

[108] M. Flechl, R. Klees, M. Kramer, M. Spira and M. Ubiali, Improved cross-section predictions for heavy charged Higgs boson production at the LHC, Phys. Rev. D 91 (2015) 075015 [arXiv: 1409.5615].

[109] Higgs Working Group collaboration, K.A. Assamagan et al., The Higgs working group: Summary report 2003, hep-ph/0406152 [INSPIRE].

[110] CMS collaboration, Search for a heavy charged Higgs boson in proton-proton collisions at $\sqrt{s}=8 \mathrm{TeV}$ with the CMS detector, CMS-HIG-13-026.

[111] S. Dawson, A. Djouadi and M. Spira, QCD corrections to SUSY Higgs production: The Role of squark loops, Phys. Rev. Lett. 77 (1996) 16 [hep-ph/9603423] [INSPIRE]. 
[112] A. Djouadi, Squark effects on Higgs boson production and decay at the LHC, Phys. Lett. B 435 (1998) 101 [hep-ph/9806315] [INSPIRE].

[113] R.V. Harlander and M. Steinhauser, Supersymmetric Higgs production in gluon fusion at next-to-leading order, JHEP 09 (2004) 066 [hep-ph/0409010] [INSPIRE].

[114] R. Harlander and M. Steinhauser, Effects of SUSY QCD in hadronic Higgs production at next-to-next-to-leading order, Phys. Rev. D 68 (2003) 111701 [hep-ph/0308210] [INSPIRE].

[115] M. Muhlleitner, H. Rzehak and M. Spira, MSSM Higgs Boson Production via Gluon Fusion: The Large Gluino Mass Limit, JHEP 04 (2009) 023 [arXiv:0812.3815] [INSPIRE].

[116] M. Carena, D. Garcia, U. Nierste and C.E.M. Wagner, Effective Lagrangian for the $\bar{t} b H^{+}$ interaction in the MSSM and charged Higgs phenomenology, Nucl. Phys. B 577 (2000) 88 [hep-ph/9912516] [INSPIRE].

[117] D. Noth and M. Spira, Higgs Boson Couplings to Bottom Quarks: Two-Loop Supersymmetry-QCD Corrections, Phys. Rev. Lett. 101 (2008) 181801 [arXiv:0808.0087] [INSPIRE].

[118] A. Djouadi and M. Drees, Higgs boson decays into light gravitinos, Phys. Lett. B 407 (1997) 243 [hep-ph/9703452] [INSPIRE].

[119] J.F. Gunion and H.E. Haber, Two-body Decays of Neutralinos and Charginos, Phys. Rev. D 37 (1988) 2515 [INSPIRE].

[120] A. Djouadi, J. Kalinowski and P.M. Zerwas, Exploring the SUSY Higgs sector at $e^{+} e^{-}$ linear colliders: A Synopsis, Z. Phys. C 57 (1993) 569 [INSPIRE].

[121] A. Djouadi, P. Janot, J. Kalinowski and P.M. Zerwas, SUSY decays of Higgs particles, Phys. Lett. B 376 (1996) 220 [hep-ph/9603368] [INSPIRE].

[122] A. Djouadi, J. Kalinowski, P. Ohmann and P.M. Zerwas, Heavy SUSY Higgs bosons at $e^{+} e^{-}$linear colliders, Z. Phys. C 74 (1997) 93 [hep-ph/9605339] [InSPIRE].

[123] ATLAS collaboration, Search for direct production of charginos and neutralinos in events with three leptons and missing transverse momentum in $\sqrt{s}=8 \mathrm{TeV}$ pp collisions with the ATLAS detector, JHEP 04 (2014) 169 [arXiv: 1402.7029] [inSPIRE].

[124] CMS collaboration, Searches for electroweak production of charginos, neutralinos and sleptons decaying to leptons and $W, Z$ and Higgs bosons in pp collisions at $8 \mathrm{TeV}$, Eur. Phys. J. C 74 (2014) 3036 [arXiv:1405.7570] [InSPIRE].

[125] T.A.W. Martin and D. Morrissey, Electroweakino constraints from LHC data, JHEP 12 (2014) 168 [arXiv: 1409.6322] [INSPIRE].

[126] WMAP collaboration, G. Hinshaw et al., Nine-Year Wilkinson Microwave Anisotropy Probe (WMAP) Observations: Cosmological Parameter Results, Astrophys. J. Suppl. 208 (2013) 19 [arXiv:1212.5226] [inSPIRE].

[127] Planck collaboration, P.A.R. Ade et al., Planck 2013 results. XVI. Cosmological parameters, Astron. Astrophys. 571 (2014) A16 [arXiv:1303.5076] [INSPIRE].

[128] J.L. Lopez, D.V. Nanopoulos and K.-j. Yuan, Accurate neutralino relic density computations in supergravity models, Phys. Rev. D 48 (1993) 2766 [hep-ph/9304216] [INSPIRE].

[129] P. Nath and R.L. Arnowitt, Predictions in SU(5) supergravity grand unification with proton stability and relic density constraints, Phys. Rev. Lett. 70 (1993) 3696 [hep-ph/9302318] [INSPIRE]. 
[130] M. Drees and A. Yamada, A decisive test of superstring inspired $E_{6}$ models, Phys. Rev. D 53 (1996) 1586 [hep-ph/9508254] [INSPIRE].

[131] L. Roszkowski, R. Ruiz de Austri and T. Nihei, New cosmological and experimental constraints on the CMSSM, JHEP 08 (2001) 024 [hep-ph/0106334] [INSPIRE].

[132] A.B. Lahanas and V.C. Spanos, Implications of the pseudoscalar Higgs boson in determining the neutralino dark matter, Eur. Phys. J. C 23 (2002) 185 [hep-ph/0106345] [INSPIRE].

[133] A. Djouadi, M. Drees and J.L. Kneur, Constraints on the minimal supergravity model and prospects for SUSY particle production at future linear $e^{+} e^{-}$colliders, JHEP 08 (2001) 055 [hep-ph/0107316] [INSPIRE].

[134] H. Baer, A. Belyaev, T. Krupovnickas and A. Mustafayev, SUSY normal scalar mass hierarchy reconciles $(g-2)_{\mu}, b \rightarrow s \gamma$ and relic density, JHEP 06 (2004) 044 [hep-ph/0403214] [INSPIRE].

[135] A. Djouadi, M. Drees and J.-L. Kneur, Neutralino dark matter in mSUGRA: Reopening the light Higgs pole window, Phys. Lett. B 624 (2005) 60 [hep-ph/0504090] [INSPIRE].

[136] G. Bélanger, F. Boudjema, A. Pukhov and A. Semenov, MicrOMEGAs_3: A program for calculating dark matter observables, Comput. Phys. Commun. 185 (2014) 960 [arXiv: 1305.0237] [INSPIRE].

[137] M. Muhlleitner, A. Djouadi and Y. Mambrini, SDECAY: A Fortran code for the decays of the supersymmetric particles in the MSSM, Comput. Phys. Commun. 168 (2005) 46 [hep-ph/0311167] [INSPIRE].

[138] CMS collaboration, Search for a standard model like Higgs boson in the decay channel $H \rightarrow Z Z \rightarrow \ell^{+} \ell^{-} q \bar{q}$ at $C M S$, CMS-PAS-HIG-12-024.

[139] ATLAS collaboration, A search for resonant Higgs-pair production in the b $\bar{b} b \bar{b}$ final state in pp collisions at $\sqrt{s}=8 \mathrm{TeV}$, ATLAS-CONF-2014-005 (2014).

[140] CMS collaboration, Search for di-Higgs resonances decaying to 4 bottom quarks, CMS-PAS-HIG-14-013.

[141] CMS collaboration, 2HDM scenario, $H$ to hh and A to Zh, CMS-PAS-HIG-13-025.

[142] K.J.F. Gaemers and F. Hoogeveen, Higgs Production and Decay Into Heavy Flavors With the Gluon Fusion Mechanism, Phys. Lett. B 146 (1984) 347 [INSPIRE].

[143] D. Dicus, A. Stange and S. Willenbrock, Higgs decay to top quarks at hadron colliders, Phys. Lett. B 333 (1994) 126 [hep-ph/9404359] [INSPIRE].

[144] S. Moretti and D.A. Ross, On the top-antitop invariant mass spectrum at the LHC from a Higgs boson signal perspective, Phys. Lett. B 712 (2012) 245 [arXiv:1203.3746] [INSPIRE].

[145] W. Bernreuther, M. Flesch and P. Haberl, Signatures of Higgs bosons in the top quark decay channel at hadron colliders, Phys. Rev. D 58 (1998) 114031 [hep-ph/9709284] [INSPIRE].

[146] V. Barger, T. Han and D.G.E. Walker, Top Quark Pairs at High Invariant Mass: A Model-Independent Discriminator of New Physics at the LHC, Phys. Rev. Lett. 100 (2008) 031801 [hep-ph/0612016] [INSPIRE].

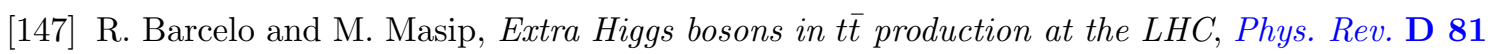
(2010) 075019 [arXiv: 1001.5456] [INSPIRE].

[148] T. Figy and R. Zwicky, The other Higgses, at resonance, in the Lee-Wick extension of the Standard Model, JHEP 10 (2011) 145 [arXiv:1108.3765] [INSPIRE]. 
[149] R. Frederix and F. Maltoni, Top pair invariant mass distribution: A Window on new physics, JHEP 01 (2009) 047 [arXiv:0712.2355] [INSPIRE].

[150] T. Plehn, G.P. Salam and M. Spannowsky, Fat Jets for a Light Higgs, Phys. Rev. Lett. 104 (2010) 111801 [arXiv:0910.5472] [InSPIRE].

[151] ATLAS collaboration, Search for $t \bar{t}$ resonances in the lepton plus jets final state with ATLAS using $4.7 \mathrm{fb}^{-1}$ of pp collisions at $\sqrt{s}=7 \mathrm{TeV}$, Phys. Rev. D 88 (2013) 012004 [arXiv: 1305.2756] [INSPIRE].

[152] CMS collaboration, Searches for new physics using the t $\bar{t}$ invariant mass distribution in $p p$ collisions at $\sqrt{s}=8 \mathrm{TeV}$, Phys. Rev. Lett. 111 (2013) 211804 [arXiv:1309.2030] [INSPIRE].

[153] A. Djouadi et al., in preparation.

[154] M. Czakon and A. Mitov, Top++: A Program for the Calculation of the Top-Pair Cross-Section at Hadron Colliders, Comput. Phys. Commun. 185 (2014) 2930 [arXiv:1112.5675] [INSPIRE].

[155] S. Kretzer, H.L. Lai, F.I. Olness and W.K. Tung, CTEQ6 parton distributions with heavy quark mass effects, Phys. Rev. D 69 (2004) 114005 [hep-ph/0307022] [INSPIRE].

[156] M. Czakon, P. Fiedler and A. Mitov, Total Top-Quark Pair-Production Cross section at Hadron Colliders Through $O\left(\alpha_{S}^{4}\right)$, Phys. Rev. Lett. 110 (2013) 252004 [arXiv:1303.6254] [INSPIRE].

[157] J. Alwall, M. Herquet, F. Maltoni, O. Mattelaer and T. Stelzer, MadGraph 5: Going Beyond, JHEP 06 (2011) 128 [arXiv:1106.0522] [INSPIRE]. 